\title{
L'evento del Vajont nei suoi aspetti geodinamici
}

\author{
P. CALOI
}

Ricevuto il 15 Aprile 1965

Rrassunto. -- Vengono riassunti i risultati di dieci anni di indagini geodinamiche (1953-1963), condotte nella stretta del Vajont preliminarmente, durante e dopo la costruzione della diga "Carlo Semenza". Si esa. minano, alla luce di essi, le probabili cause del tragico evento del 9 Ottobre 1963.

Si aggiungono brevi considerazioni sulla ripresa dell'attività sismica, a monte della diga, verificatasi nella prima decade di Settembre 1965.

SUMMARY. - The results of geodynamic investigations, in a space of ten years (1953-1963) obtained in the Vajont's gorge during and after construction of the "Carlo Semenza" dam, they are here resumed.

On their basis, it is examined the probable reasons of the October $9^{\text {th }} 1963$ tragic event.

It is taken into consideration the seismic activity's awaking-towards the eastern zone of the dam- in the first ten days of September 1965 oceurred.

\section{Premessa.}

Questo scritto si riferisce agli interventi ed agli studi che ho compiuti, in relazione con lo sbarramento del Vajont, dal 1953 sino alla catastrofe del 9 Ottobre 1963 ed anche successivamente. Tali studi e tali interventi vanno dalle prime determinazioni delli risposta elastica della roccia, nella fase di progettazione del grande manufatto, sino ai controlli di vario tipo, durante la costruzione dell'opera, ed alle rilevazioni prima e durante gli invasi sperimentali del serbatoio ed anche dopo il cataclisma. Posso dire 
che, con gli strumenti che ho installati sul posto per conto della SADE prima e dello ENET poi, ho seguito tutta la vita del manufatto e dell'ambiente fisico ove è sorto. Il che mi far avvertire quasi il dovere di portare anche un mio contributo ai tentativi che, da più parti, si vamno facendo per scoprire le cause che possono aver determinato l'immane evento. Da tali studi la scienza e la tecnica potranno trarre insegnamenti validi per l'avvenire.

Disponiamo oggi di una serie di elementi che ci permettono di dare una interpretazione di quel concervo di osservazioni ed accertamenti che al momento in cui erano raccolti non ci consentivano di intendere, se ed in qual modo, si sarebbero potuti inserire nel dinamismo di un fenomeno così complesso e così eccezionale - direi, unico - come quello poi verificatosi.

Abbiamo infatti a nostra disposizione $\mathrm{i}$ sismogrammi ottenuti a Pieve di Cadore, a Tolmezzo ed altrove, a registrazione del crollo, e delle sue conseguenze immediate. Da essi abbiamo cercato di trarre elementi atti ad illuminarci sulle cause.

Ciò che segue pertanto va considerato come un tentativo - umile tentativo - di interpretazione di un evento che, nell'entità e fulmineita del suo manifestarsi, appare - anche a fatti avvenuti - fuori di ogni umana, ragionevole possibilità di previsione.

I.

Prima delu'evento.

1. - Caratteristiche fisiche della roccia nella stretta del Fajont prima dello sbancamento.

Il mio primo intervento nella stretta del Vajont (Fig. 1 e 2), risale all'estate del 1953. Fu provocato su richiesta dell'Ufficio Studi della SADE ed ebbe per scopo la determinazione della risposta elastica della roceia, con il metodo già sperimentato in precedenza a Pieve di Cadore e presso la stretta del Lumiei, in quel di Sauris.

Non mi sofiermerò sulle modalità e le finalità del metodo, già diffusamente esposte in mie precedenti pubblicazioni (1). Dirò solo che esso si avvale del rilevamento della velocità delle onde longitudinali e trasversali, destate nel mezzo dal brillamento di piccole cariche di esplosivo. E noto che la velocità di tali onde è legata alla densità del mezzo e al 
suo modulo di elasticità (modulo di Young) $E$. Usufruendo di ur solo tipo d'onda (longitudinale o trasversale) è giuocoforza (a scanso di altre indagini collaterali) presupporre uguale a $1 / 4$ il valore del rapporto $\sigma$

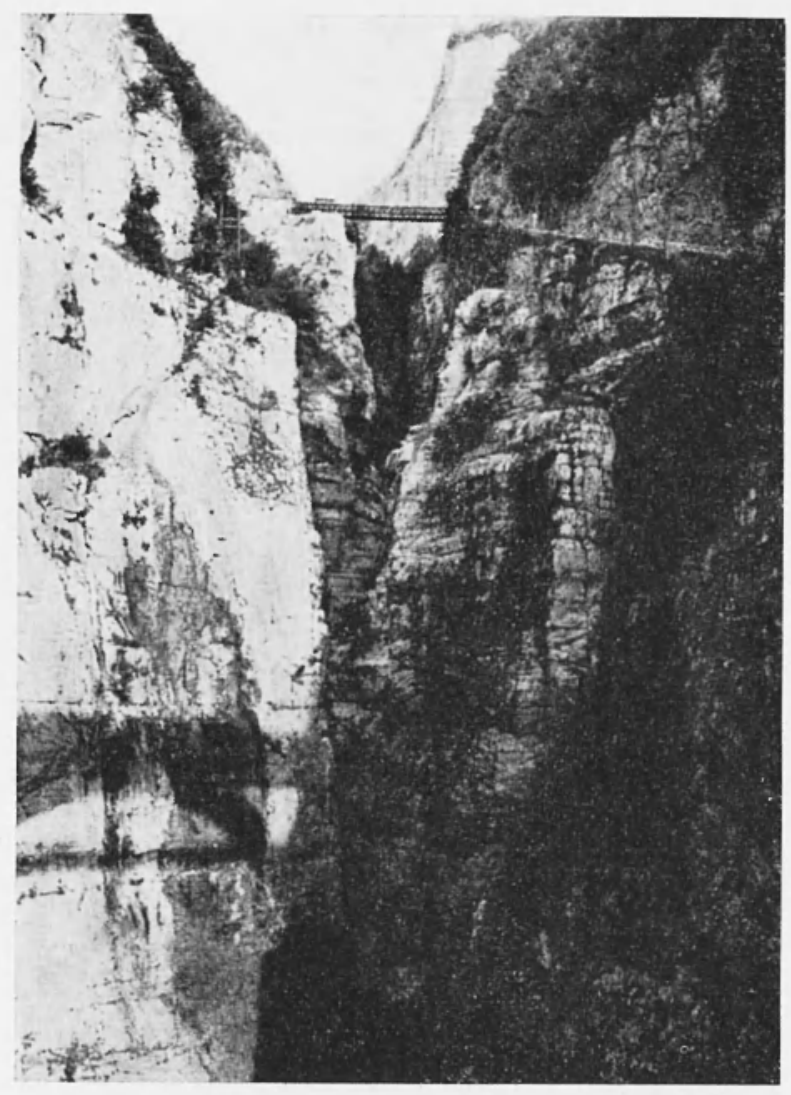

Fig. 1 - La stretta del Vajont nell'A grosto 1953 (foto Caloi), vista dal pontecanale (ombra in basso, a sinistra). Il ponte in ferro in alto, al centro, adduceva a Erto, ad una quota di poco superiore a quella del ponte-canale (590 m. ca.). Dopo la costruzione della diga (ancorata alle pareti rocciose a valle del ponte in ferro), il ponte e le poche case intorno vennero sommerse dal successivo invaso.

Sullo sfondo, in alto, oltre il ponte in ferro, in sponda sinistra sovrasta " Il Pinnacolo ", massiccio roccioso che, alle indagini del 1959 appariva ancora (salvo la parte terminale) elasticamente valido.

di Poisson. Si sa che tale rapporto varia effettivamente fra 0 e $1 / 2$; pertanto è preferibile — per avere valori di $E$ più approssimati — servirsi 
di procedimenti che prescindano dal valore di $\sigma$. A questo fine, ho ottenuto, e sovente applicato, la formula

$$
E=\varrho \frac{v_{\mathrm{n}}^{2}\left[3\left(\frac{v_{1}}{v_{2}}\right)^{2}-4\right]}{\left(\frac{v_{1}}{v_{2}}\right)^{2}-1},
$$

dove o è la densità, $v_{1}$ la velocità delle onde longitudinali e $v_{2}$ quella delle onde trasversali, proprie del mezzo.

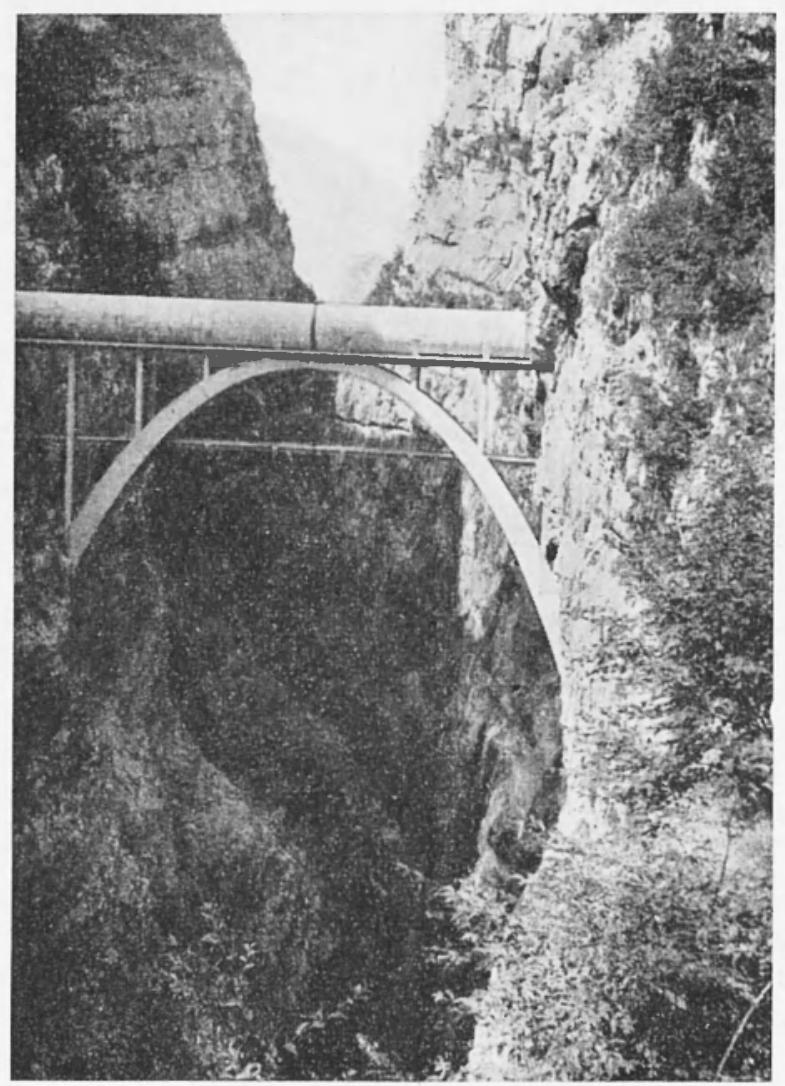

Fig. 2 - Il ponte-canale (detto anche ponte-tubo), visto da monte (foto Caloi, 1953), verso J Longarone.

Le registrazioni delle piccole esplosioni furono ottenute con uno speciale tipo di vibrografo a tre componenti, particolarmente adatto a questo scopo, in quanto, funzionando rome un autentico piccolo sismo- 
grafo, consente la regristrazione di una larga gamma di vibrazioni sismiche - trasversali comprese —, difficimente osservabili con gli abituali apparechi per prosperione sismica.

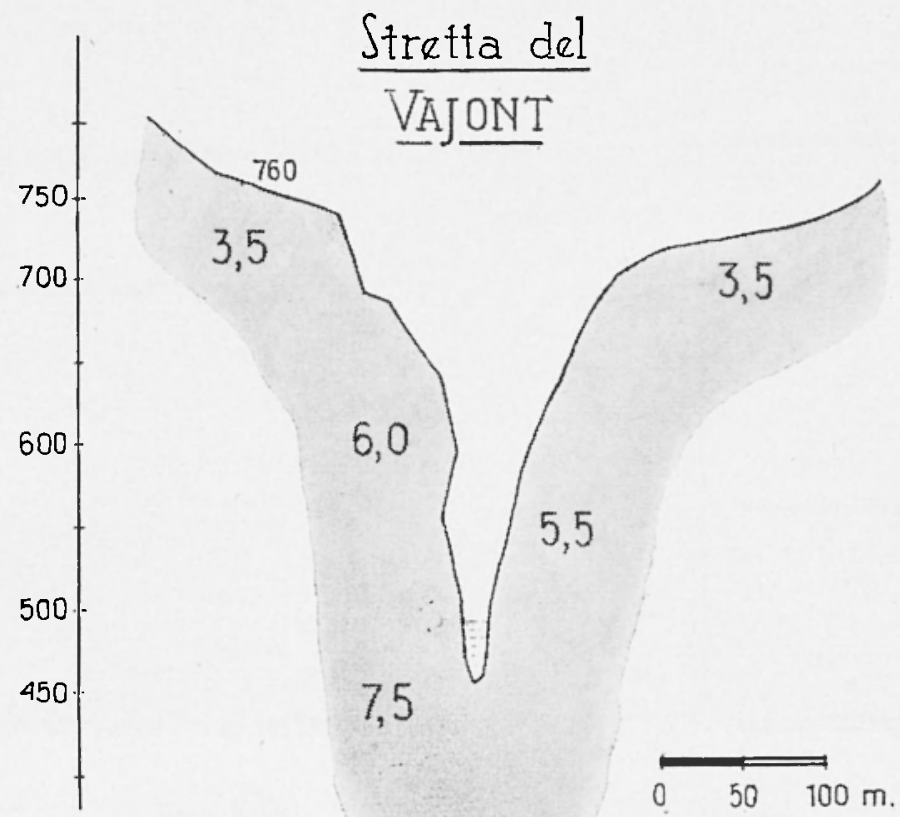

Fig. 3 - Spaccato della stretta del Vajont prima dello sbancamento. I numeri riportati lungo i bordi della valle indicano le velocitit telle onde longitudinali (in $\mathrm{km} / \mathrm{sec}$ ), ivi osservate.

La serie d'indagini eseguita nel 1953 fu limitata alla parte della valle riguardante il tratto di roccia fra il ponte canale (quota 590 circa) e il fondo. $\Lambda$ suo tempo, fu riferito alla SADE sui risultati ottenuti. Il completamento dell'esame delle caratteristiche elastiche della roceia per il tratto di valle che dal livello del ponte canale porta verso il limite superiore della valle fino a quota 725 circa, fu compiuto nell'estate del 1956. Anche di tale completamento fu data notizia all'Ufficio Studi SADE nel Novembre 1956.

Non posso qui scendere a particolari. Come osservazione generale risultò che, nel suo complesso, la roccia in spalla destra presentava caratteristiche di compattezza molto più accentuate - specie per quanto si riferisce al tratto che dal ponte canale va al fondo valle - di quella 

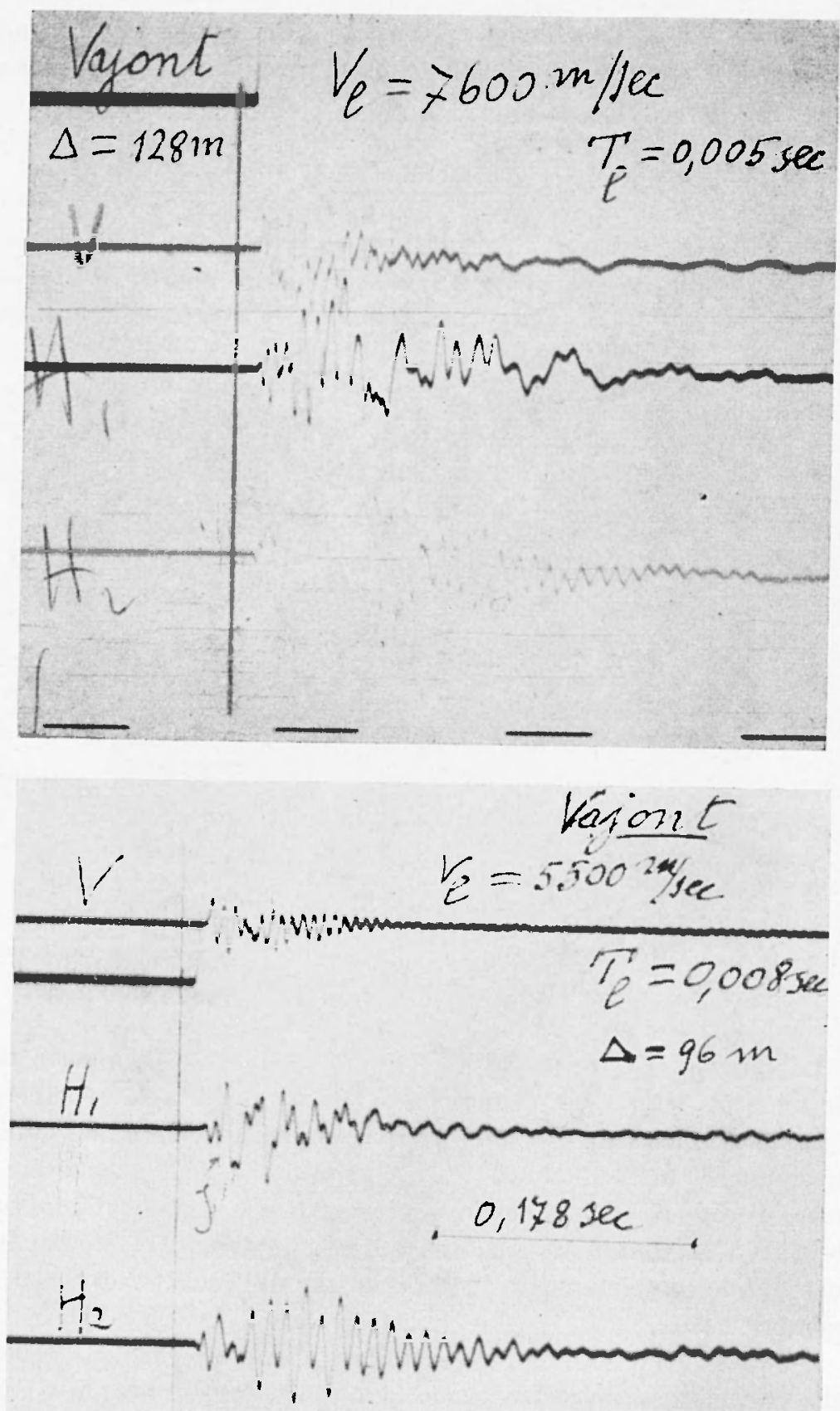

Fig. 4 

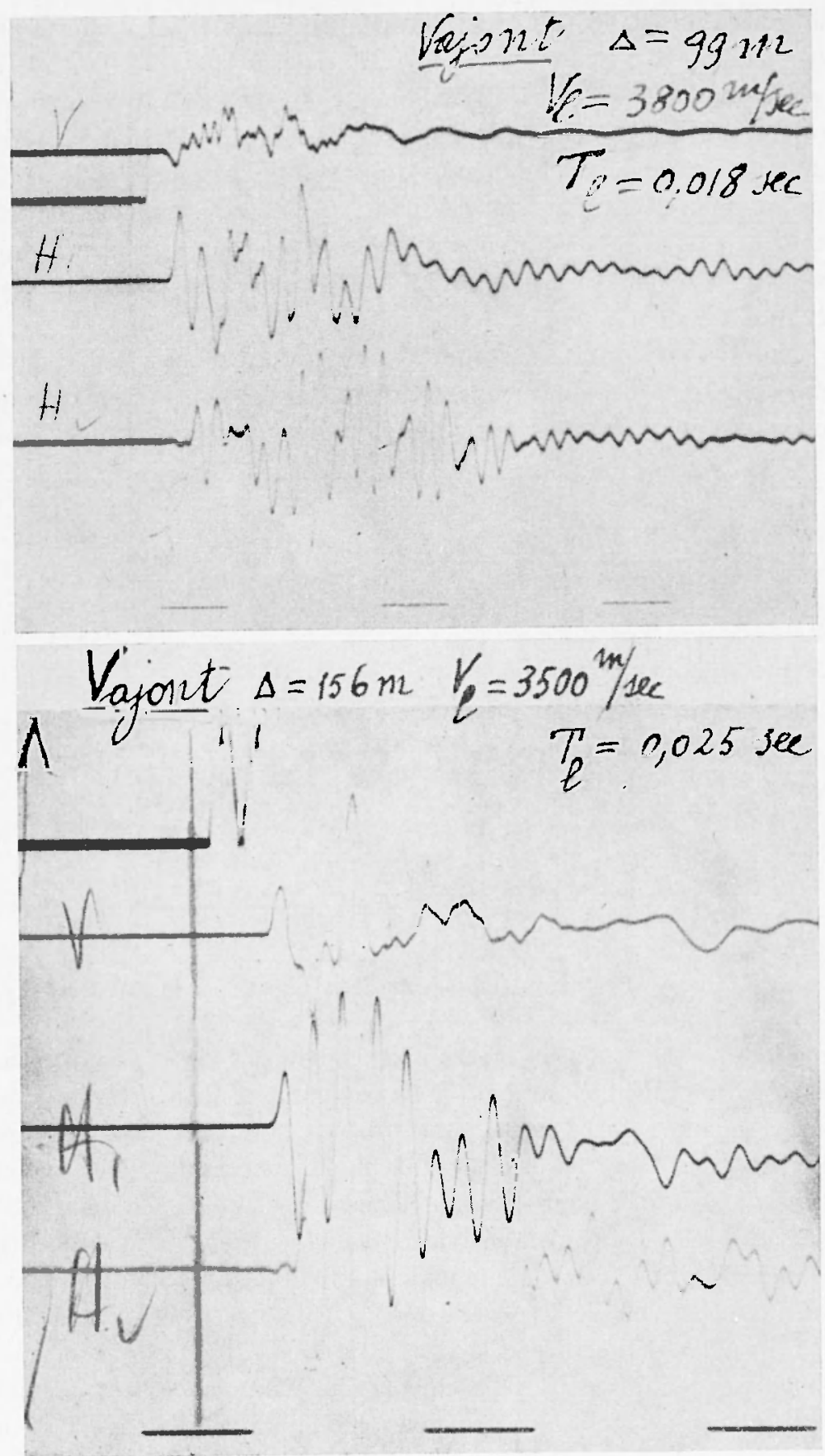

Fig. $4 a$ 
della roccia in spalla sinistra: quest'ultima presentava più marcata omogeneità.

Per quanto si riferisce all'andamento della velocità, a conferma di quanto si era già ottenuto per Pieve di Cadore, i valori medi di questa grandezza andavano gradualmente diminuendo dal fondo valle al limite superiore della zona indagata, passando, per quanto concerne la sponda destra, da valori di $7,5 \mathrm{~km} / \mathrm{sec}$ sul fondo, a $5,5-5,0 \mathrm{~km} / \mathrm{sec}$ (intorno alle quote 590-680), per terminare a $3,5 \mathrm{~km} / \mathrm{sec}$ (a nella parte più elevata (Fig. 3).

Riusciva inoltre manifesto un altro fatto; e cioè che la diversità del mezzo attraversato - indipendentemente dal modulo - risultava qualitativamente espressa dalla forma dei vibrogrammi ottenuti, nel senso che

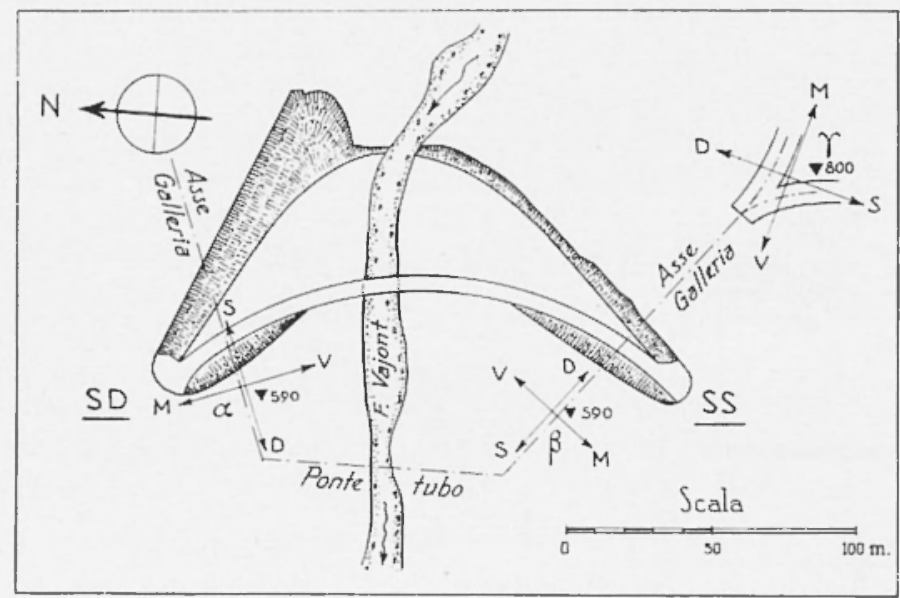

Fig. 5 - Postazioni clinografiche a quota ponte-canale.

il graduale passaggio da un mezzo ad un altro via via più compatto, era rivelato da un sensibile aumento della frequenza delle vibrazioni longitudinali (Figg. 4 e $4 a$ ). Questa constatazione - controllata successivamente in numerose altre esperienze (al Glagnò, a Zermula sull'alto Chiarzò, a Pieve di Cadore, a Ponte Foos, nella valle del Maè, in Vallarsa, sull'alto Travignolo, ecc.) - mi consentiva di scoprire il fenomeno della dispersione anomala, nell'ambito delle onde sismiche di altissima frequenza( $\left.{ }^{1}\right)$.

Un aspetto complessivo della roceia nella stretta del Vajont, prima dell'inizio dello sbancamento, è riassunto nella Fig. 3, in cui le graduali attenuazioni dell'intensità della colorazione (nero su bianco) dà un'idea della attenuazione della durezza della roccia, quando si passa da oltre $10^{6} \mathrm{~kg} / \mathrm{cm}^{2}$ (sul fondo valle) a circa $3,3.10^{5} \mathrm{~kg} / \mathrm{cm}^{2}$ (a quota $725 \mathrm{ca}$ ). 

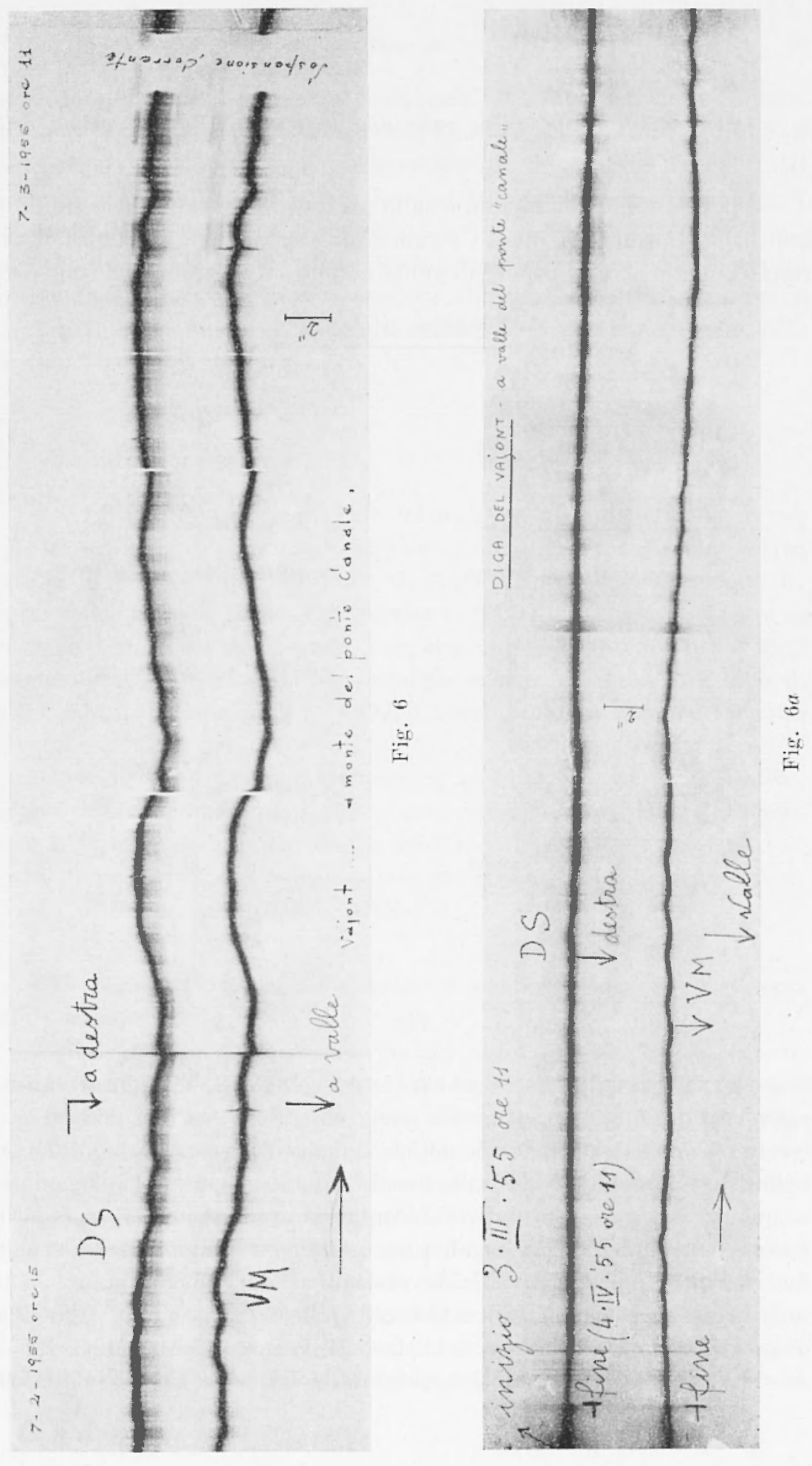
2. - Osservazioni clinografiche sulle due sponde del Vajont, prima dello sbancamento.

Sull'uso del fotoclinografo e sulle preziose - spesso insostituibili indicazioni fornite da questo strumento, ebbi ad intrattenermi, a più riprese, nel corso di relazioni e di pubblicazioni sul controllo delle dighe $\left.{ }^{(}\right)$.
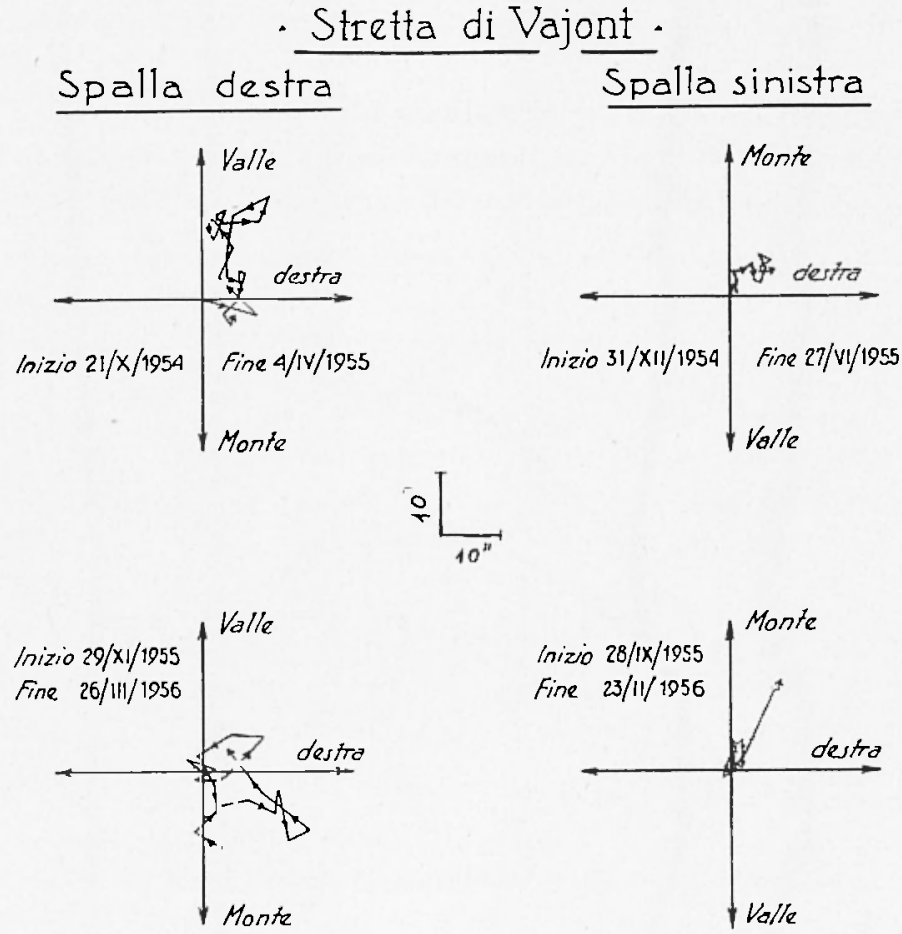

Fig. 7

Qui dirò soltanto che, fra i problemi la cui soluzione viene notevolmente agevolata dal fotoclinografo, vi è quello di stabilire se una determinata località è oppure no, partecipe dei movimenti di un solo tratto di erosta terrestre (1). È evidente l'interesse legato a questa risposta. Analogamente a quanto era già stato fatto all'Ambiesta a tale scopo furono poste due stazioni clinografiche, in galleria a quota ponte-canale, una in sponda destra l'altra in sinistra (Fig. 5), stazioni che funzionarono, pressoché inin terrottamente, dall'Ottobre 1954 all' $A$ prile del 1956. I risultati dell'indagine riuscirono quanto mai soddisfacenti. Prima dell'inizio dei lavori di scavo, i fotoclinografi non rivelarono infatti variazioni apprezzabili della 
verticale apparente e le piccole deviazioni osservate potevano ragionevolmente essere attribuite ai micromovimenti della crosta, più che al contrasto di due limitrofi blocchi geodetici. Le Figg. 6, 6a danno tratti di clinogramma, ottenuti presso le predette stazioni, mentre la Fig. 7 sintetizza l'andamento delle risultanti.

All'inizio dei lavori di scavo (1956), a motivo delle perturbazioni provocate dalle esplosioni, le due stazioni clinografiche furono rimosse e rimasero inattive fino al completamento delle trincee di ancoraggio della costruenda diga.

\section{3. - Lavori di sbancamento.}

Nel frattempo, avevano avuto inizio le operazioni di sbancamento della roccia, nella zona destinata allo sbarramento. Le cariche d'esplosivo fatte brillare suscitarono uno stato di apprensione nella popolazione del soprastante paese di Casso. Ciò indusse la SADE a richiedere misure di controllo degli spostamenti e delle accelerazioni ivi destate. Le prime registrazioni eseguite portarono alla conclusione che le vibrazioni erano di entità del tutto trascurabile ai fini del benché minimo danneggiamento agli edifici.

Altre registrazioni furono effettuate in Casso (presso la canonica), successivamente, a seguito di una grossa esplosione provocata il 25 Febbraio 1957 nella sottostante stretta del Vajont.

A proposito di tale esplosione, ecco parte della relazione a suo tempo inviata all'Ufficio Studi della SADE:

"La postazione per la registrazione è stata sistemata al piano terra della Canonica di Casso.

I sismogrammi ottenuti sono risultati vistosi, fun dall'inizio. Infatti, a differenza di quanto si ̀̀ verificato nelle registrazioni precedenti, gli spostamenti reali del suolo corrispondenti alle onde longitudinali, sono stati ampi, su tutte e tre le componenti: $\mu 6$ per la componente verticale, $\mu 1.8$ per una componente orizzontale, $\mu 4.8$ per l'altra componente orizzontale a $90^{\circ}$.

La risultante spaziale è stata quindi di circa $8 \mu$.

Poiché il periodo associato a quest'onda era di 0.0\%6 sec, la corrispondente accelerazione ha assunto il valore di $5.5 \mathrm{gal}$ ca.

Ben più ampio è risultato il movimento reale del suolo corrispondente all'arrivo delle onde trasversali. Si sono avuti infatti, $34 \mu$ per la componente orizzontale $\left(H_{1}\right), 51 \mu$ per l'altra componente orizzontale $\left(H_{2}\right)$ e $1 \% .5 \mu$ per la 
componente verticale. Nello spazio si è avuto quindi, in corrispondenza delle onde trasversali, uno spostamento complessivo di circa $6 \pm \mu$. Poiché il periodo proprio delle onde trasversali è risultato di 0.17 sec, la corrispondente acclerazione ha avuto un valore di $9 \mathrm{gal}$. ca. L L a dove il movimento ha raggiunto i massimi spostamenti è stato in corrispondenza delle onde di flessione. Queste oscillazioni si sono manifestate in un lungo seguito, raggiungendo massimi che sono andati ripetutamente ben oltre il campo di registrazione, rendendo pertanto l'interpretazione solo approssimativa. Ad ogni modo, si sono potuti rilevare i seguenti spostamenti del suolo in corrispondenza del massimo delle oscillazioni: e precisamente $55 \mu$ per l'orizzontale $\left(B_{1}\right)$ e $83 \mu$ per l'orizzonlale $\left(H_{2}\right)$ lè noto che le onde di flessione, in casi come questi, non hanno praticamente componente verticale]. Ciò porta ad uno spostamento orizzontale complessivo di $100 \mu$ ca. Il periodo di dette oscillazioni è risultato di 0.19 sec; pertanto la corrispondente accelerazione ha superato $i 12$ gal.

A conclusione di quanto sopra, va rilevato che la pericolosità di queste ultime oscillazioni (e anche di quelle trasversali) non è tanto dovuta all'entità degli spostamenti e delle accelerazioni destate, quanto al periodo al quale le oscillazioni stesse sono associate, periodo che è stato dell'ordine di 0.2 sec. Tenendo presente che tale periodo è predominante nelle strutture di piccole abitazioni, è chiaro che possono seguirne fenomeni di risonanza, a carattere qualche volta distruttivo; tanto più che, nel caso specifico, le oscillazioni ai flessioni sono durate ininterrotte per circa 2 sec, e la registrazione è stata fatta al piano terra.

La carica di esplosivo di cui si è fatto uso il 25. Febbraio, risulta pertanto eccessiva; ripetuta più volte, essa potrebbe senz'altro diventare dannosa ".

A complemento di quanto sopra va aggiunto ehe la arica esplosiva risultante, pressocethé in parti uguali, di "OD1MI" e "Dinamon", fu di $\mathrm{kg} 2276$.

La distanza del punto di scoppio, in sponda destra, dal punto di registrazione fu di $m 630$.

4. - Ripetizione delle indagini geosismiche nella roccia dimposta della diga del Vajont a sbancamento ultimato, in zone allo stato naturale o già iniettate.

Nel 1957, furono eseguiti altri controlli del modulo di elasticità della rocoia, nel trincerone di ancoraggio della diga.

Nelle parti piì interessate dal brillamento degli esplosivi, l'elasticita risultò aver subito qualche flessione più o meno sensibile, sia a causa 
della decompressione conseguente al distac'o di bancate roceiose, sia come conseguenza delle sollecitazioni rui la rorecia era stata sottoposta dall'azione delle esplosioni. Tuttavia le registrazioni non testimoniarono

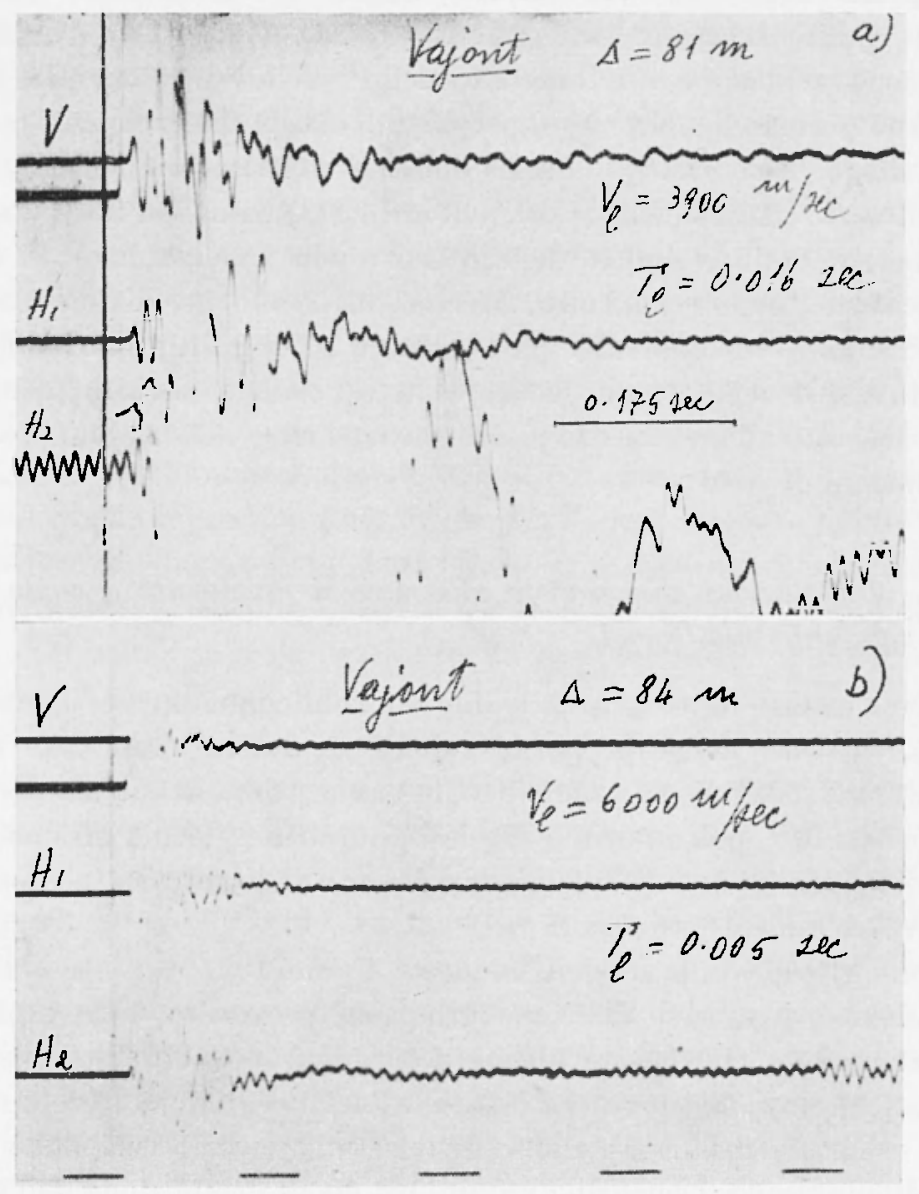

Fig. 8 - Vihrogrammi di una stessa zona, prima e dopo l'iniezione cementizia (si osservi il notevole ammento della frepuenza delle onde elastiche dopo l'iniezione).

l'esistenza di estese discontinuità. Soltanto a circa 170 metri dalla superfieie esterna della valle si notarono echi da una superficie riflettente, con andamento approssinativo analogo a quelio della fianeata della valle stessa, senza peraltro fosse dato di conoscere l'estensione di tale superficie, che semblava limitata alla parte superiore della valle. Nelle zone 
iniettate, la rigidità della roccia - come già rilevato in precedenza appariva pur sempre elevatissima (Fig. 8).

Le indagini furono estese nel Dicembre 1957 e vennero ulteriormente ripetute nel Maggio 1959, nell'Aprile, Luglio ed Agosto 1961.

Da queste successive indagini risultò che i valori elevatissimi, ai quali portava inizialmente il modulo della roccia l'iniezione di cemento, andavano scemando col tempo, per assumere alla fine valori di poco più piccoli di quelli originari, ma sensibilmente superiori a quelli riscontrati subito dopo lo sbancamento. Cosi, nell'ultima indagine del 1961, il modulo di Young, dell'ordine di $6.10^{5} \mathrm{~kg} / \mathrm{cm}^{2}$ sul fondo valle, andava, in destra, lievemente flettendo verso l'alto, dove, in corrispondenza del coronamento diga, risultava mediamente pari a $3-3,5.10^{5} \mathrm{~kg} / \mathrm{cm}^{2}$; la dimiriuzione presentavasi lievemente più accentuata in sponda sinistra, conformemente del resto all'andamento riscontrato nei rilievi che hanno preceduto le operazioni di scavo.

5. - Le due stazioni clinografiche ritornano a funzionare a quota pontecanale, sulle due sponde.

Come è stato detto al n. 2, le due stazioni clinografiche sistemate in nicchie, ricavate nella roccia, in gallerie di destra e sinistra rispettivamente, verso la fine del 1954, oltre due anni prima dell'inizio dei lavori di sbancamento, non avevano rivelato, durante il loro funzionamento, che movimenti lievissimi, quali normalmente si registrano in posti della Terra relativamente tranquilli.

Come si è detto, le stazioni suddette furono tolte durante i lavori di sbancamento, a motivo delle perturbazioni provocate dalle esplosioni. i stato provato che queste ultime determinarono alterazioni nelle caratteristiche elastiche, dovute al fatto che l'azione dirompente delle cariche non si esaurisce nell'asportazione di quantitativi di roccia più o meno notevoli, ma provoca pure una minuta azione di demolizione dell'edificio molecolare del mezzo elastico e il conseguente aumento della porosità $\left({ }^{2}\right)$ Il campo delle tensioni interne ne risulta turbato. Al Vajont, questa alterazione d'equilibrio ebbe a manifestarsi entro le gallerie, e all'aperto, con brontolii, piccoli schianti, accompagnati talvolta da caduta di frammenti di roccia. Questi fenomeni culminarono in uno schianto fragoroso (estate 1958), inteso in tutta la valle. Dopo una breve sospensione dei lavori, questi avvertimenti suggerirono l'opportunità di rimettere in funzione le stazioni clinografiche, allo scopo di controllare - nei limiti del possibile - i fenomeni accennati. Ciò avvenne verso la fine di Agosto 1958. 
Non ostante gli apparecchi fossero stati rimessi nelle condizioni di sensibilità minima, essi sorpresero in atto notevoli movimenti di rotazione, tali da provocare la fuoruscita dell'immagine luminosa più volte nello stesso giorno (Fig. 9 e 10).

Questi movimenti non si sono sempre verificati nella stessa direzione, anche se sembravano seguire in media una direzione prevalente; e non furono nemmeno caratterizzati da uniformità di morimento, in quanto a periodi di agitazione si alternavano periodi di calma. In concomitanza con questa inquietudine, un altro fatto testimoniava un certo lavorio di forze contrastanti nella roccia: la frequente rottura dei fili di sospensione dei pendolini clinografici. Quale provvisoria spiegazione, ho ritenuto di poter attribuire eventi del genere all'azione dirompente di piccoli schianti nella roccia, che generalmente passavano inosservati perché la loro fulmineità provocava vibrazioni nel campo degli ultrasuoni.

Ritenni che le anormali ondulazioni osservate fossero da attribuire all'azione di decompressione, conseguente all'alleggerimento provocato nel sistema roccioso dagli scavi e dagli effetti delle esplosioni. La decompressione si traduceva in una "caduta", verso l'asse dalla valle, delle due pareti, resa complicata dall'eterogeneità dei materiali e dalla resistenza dei blocchi limitrofi.

Di qui il verificarsi di moti torcenti, sovrapposti a quelli di fondo.

Va ancora rilevato che le due postazioni clinografiche si trovavano presso zone profondamente iniettate. Le iniezioni hanno certamente contribuito alla inquietudine di dette zone, tendenti successivamente verso nuove posizioni di equilibrio. La quantità di cemento iniettata, infatti, è stata sufficiente ad alterare profondamente le caratteristiche elastiche della roccia, tanto da raddoppiarne o triplicarne addirittura il modulo di Young. Si è venuto così a formare un nuovo mezzo con caratteristiche proprie, che finì coll'uniformarsi alla statica precedente, sia pure dando prima luogo a perturbazioni dinamiche più o meno manifeste.

La Fig. 11 mette in piena evidenza l'entità degli spostamenti clinografici osservati, e la loro ampiezza; specie se paragonata a quella dei moti registrati prima dell'inizio dei lavori.

Nell'Agosto del 1959 ritenni di poter attribuire il fenomeno delle variazioni clinografiche come dovuto a tre cause: sbancamento delle rocee con esplosioni, iniezioni di cemento e costruzione della diga, che tendeva a riportare il complesso roccioso verso nuove posizioni di equilibrio.

Tali fenomeni andarono via via scemando, a mano a mano che la diga - la cui costruzione, nel frattempo era stata iniziata - cominciò a prendere quota; tanto che nel Settembre 1959 la crisi poteva local- 
mente ritenersi superata, avendo il sistema raggiunto nuove posizioni di equilibrio. is da ritenere che la diga abbia avuto, al riguardo, ma funzione riequilibrante.

Nella fase sopra descrita, al Vajont non funzionava ancoma la stazione sismica. Verso la finc del 1959, nella cabina comandi, in sponda sinistra, fu sistemata una terna di fotosismografi "Girlanda ", ad amplificazione elettromagnetica, formanti nell'insieme ma stazione sismiea di primissino ordine, per la prima volta fumzionante presso una grande liga.

6. - Indagini geosismiche eseguite in sponda sinistra, a monte della diga del Fajont, nel Novembre 1959.

Su richiesta della S.MDE (Ufficio Costruzioni Idrauliche), nel Novembre 1959 fu compiuta mal campagna geosismica nella zona a monte della diga del Vajont, in ma località dove interessava conoseere se l'ammasso roceioso in sponda sinistra si fosse formato in loco, o si dovesse considerare come parte di ma frama del Toc.

Non mi soffermerò a riportare i particolari di questa campagna, sviluppatasi lungo due profili che, da fondo-ralle a quota 553 ca, salgono il primo a quota $756(R)$ e il secondo - lievemente inclinato nei rispetti del precedente - da quota $R$ a quota $850(R R)$. In quest'ultima direzione, sono stati fatti rilievi in un senso a nel senso opposto (Fig. 12).

Riporto qui parte della relazione, inviata a suo tempo (t Febbraio 1960) all'Ufficio Studi - Venezia -.

"Prima nostra preoccupazione è stata quella di investigare la natura della roccia di fondo. A cio hanno servito le registrazioni compiute su distanze. relativamente grandi allo scopo di ridure al minimo l'azione ritardante di eventuali depositi di frana.

Il risultato non poteva essere piu convincente: infatti su tutte le convenienti distanze sperimentate, $i$ valori otten uti per la velocità sono fra i piu elevati ai tutte le compagne eseguite, paragonabili a quelli avuti, a suo tempo, sul fondo della stretta del Vajont, in zona diga.

Tisulta pertanto una roccia a modulo elastico elevatissimo (dell'ordine di $10^{6} \mathrm{~kg} / \mathrm{cm}^{2}$ ), a testimonianz: della sua compattezza. La riprova dell'estrema solidita del mezzo è data altresi dalla elevatissima frequenza delle onde longitudinali e trasversali che la interessano. 

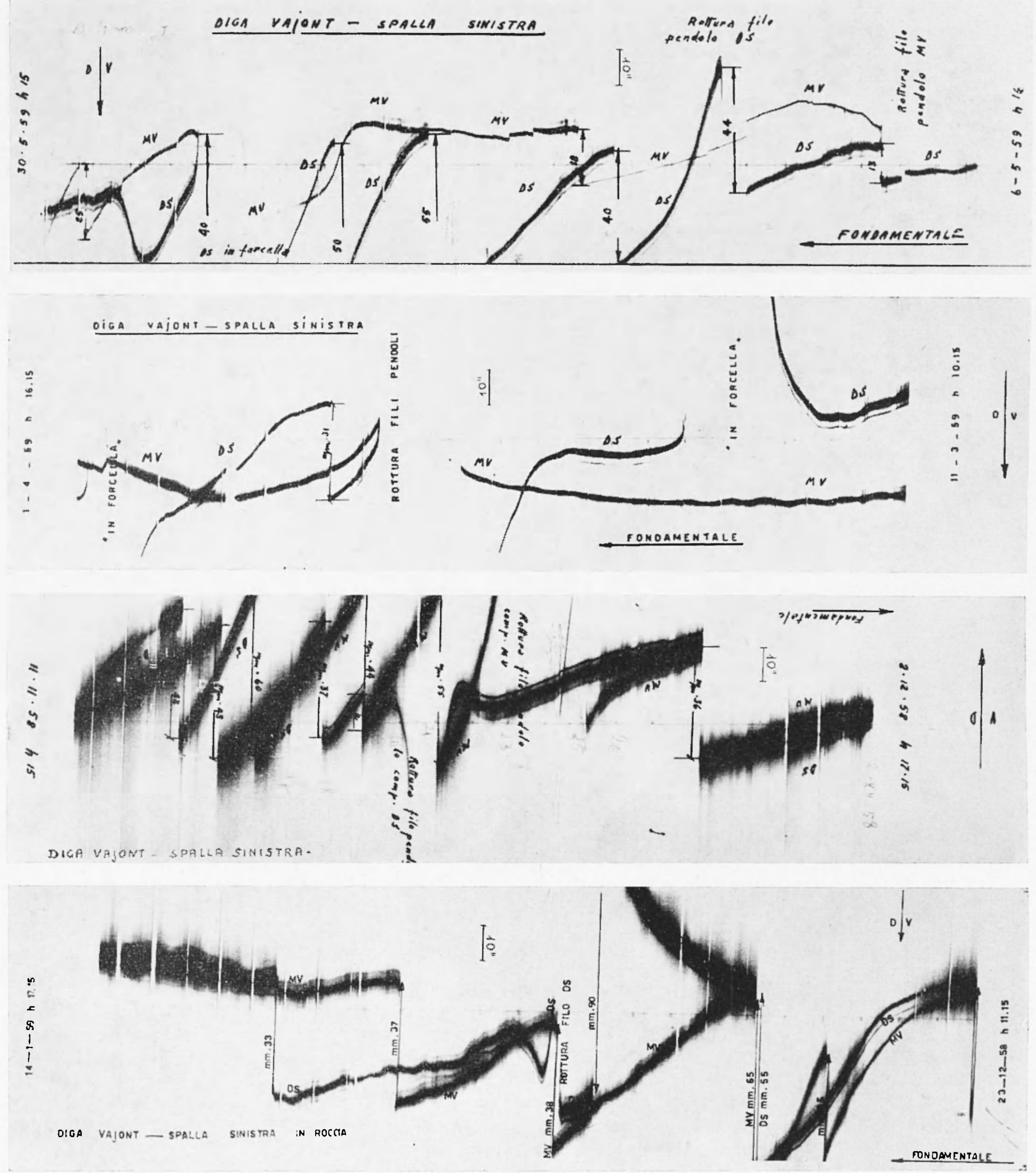

Fig. 9 - Forti rotazioni locali registrate dai clinografi dopo lo sbancamento. 
P. CaIror

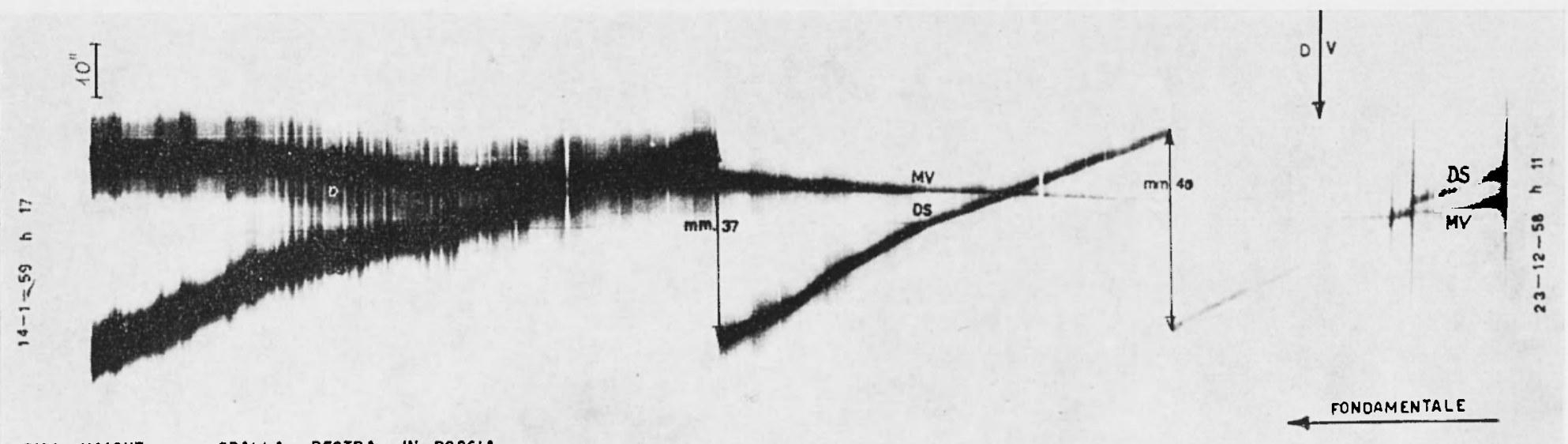

DIGA VAjONt - SPALLA DESTRA IN ROCCIA
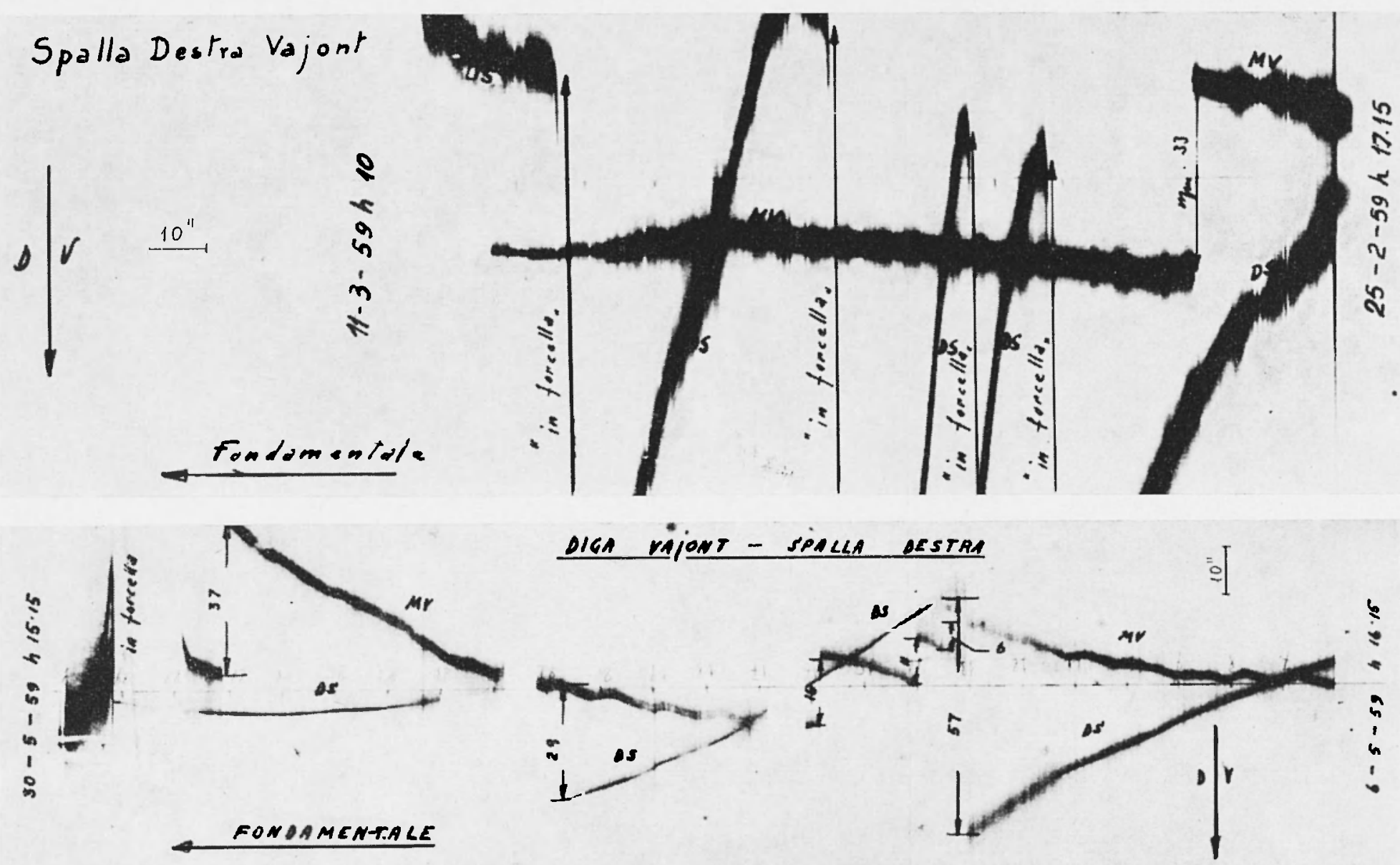

Fir. 10 - Forti rotazioni locali registrate dai clinografi dopo lo shancamento. 


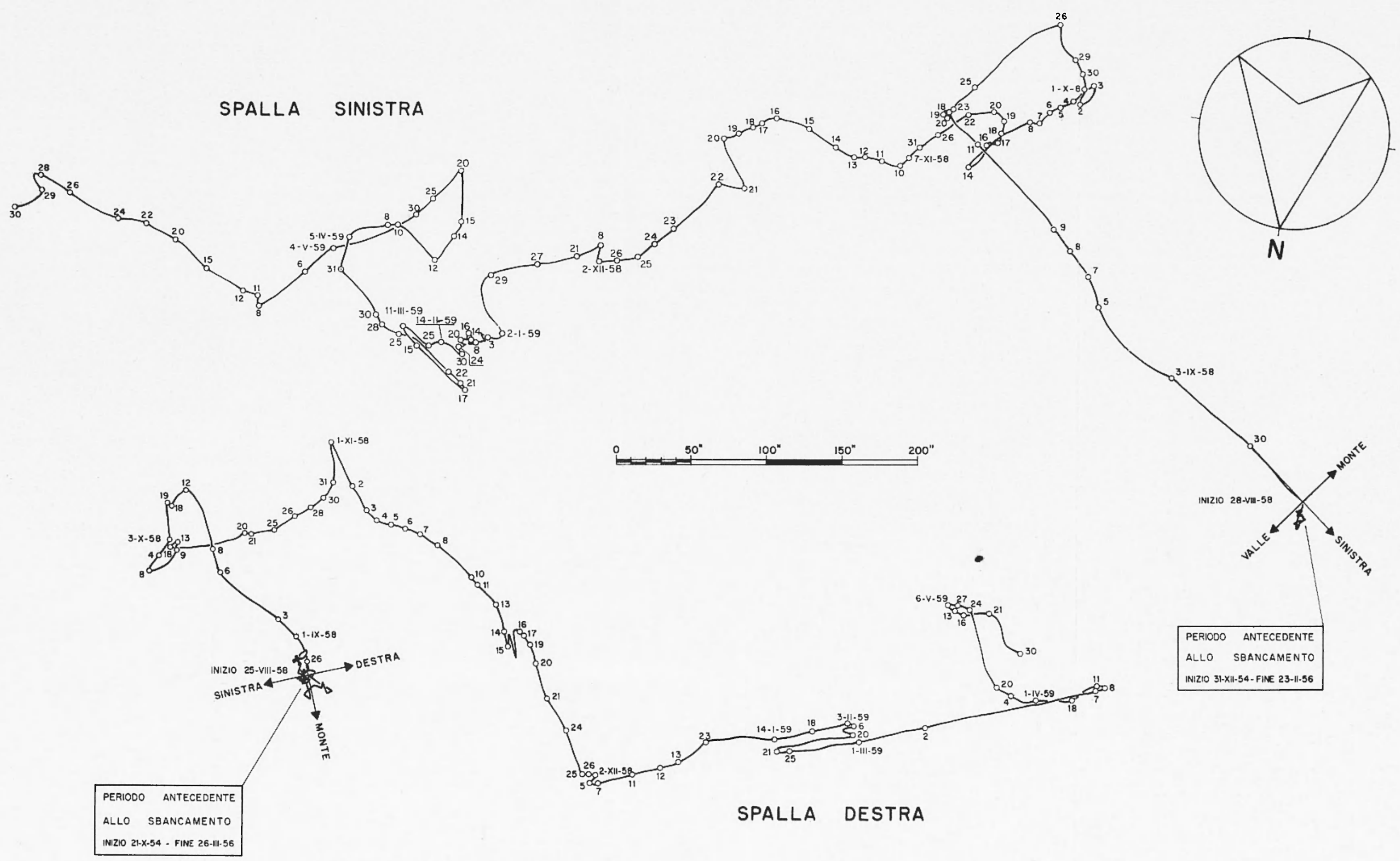

Fig. 11 - Risultati delle rotazioni della verticale apparente, nel periodo interessato alle registrazioni. 


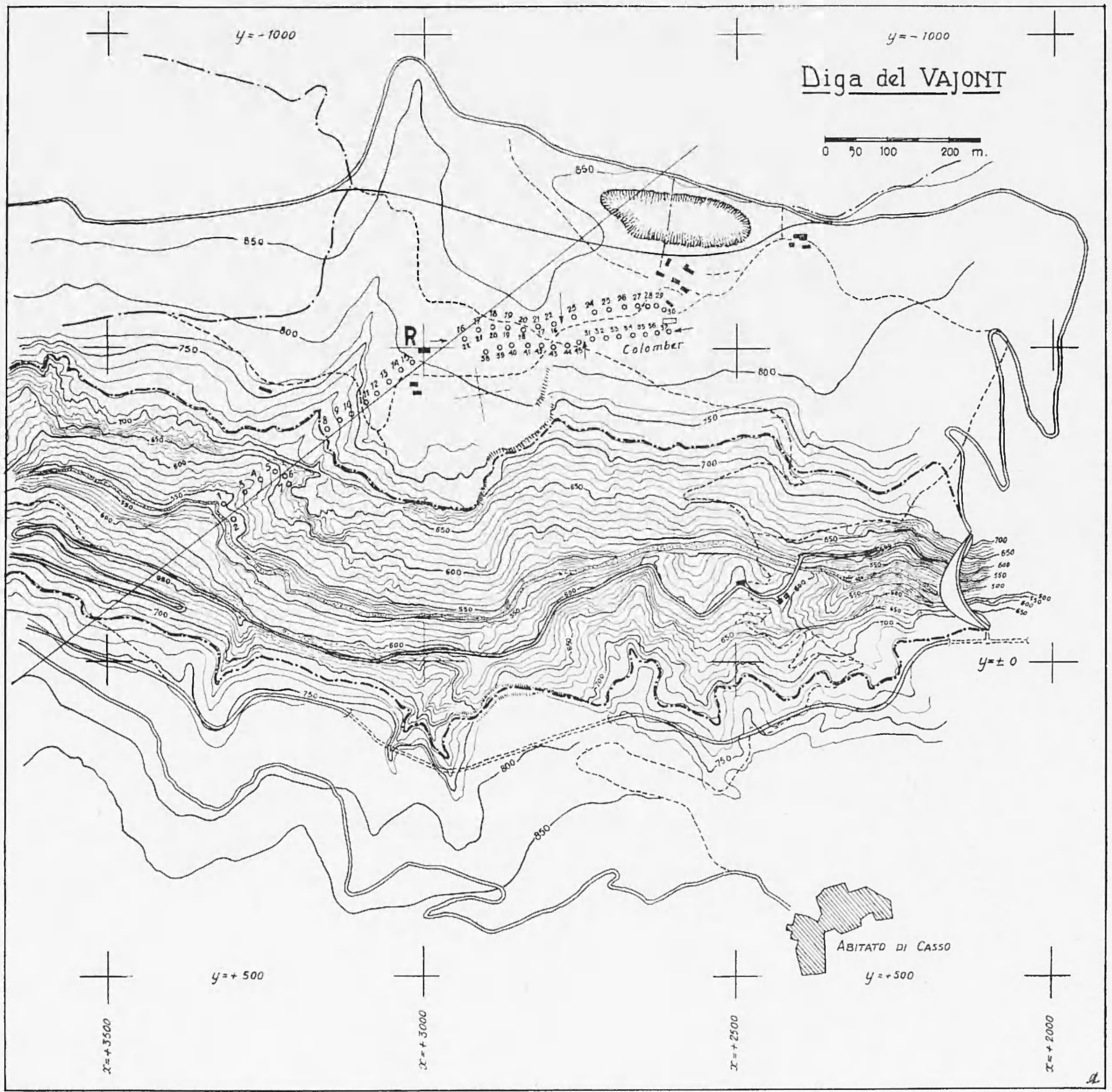

Fig. 12 - Planimetria relativa alle indagini condotte nel 1959 (Ricostruzione approssimativa). 
Ciò sta a dimostrare che il massiccio in sponda sinistra, nella zona presa in esame, si è formato in loco. Esso infatti non può essere precipitato dall'alto sotto forma di frana, perché le sue caratteristiche sarebbero risultate nettamente diverse, anche qualora, nella caduta, l'ammasso roccioso avesse conservato la sua compattezza; anche in questo caso favorevole, infatti, il suo modulo elastico sarebbe risultato inferiore a quello riscontrato.

È noto - a questo riguardo - che nelle grandi vallate il modulo di Young della roccia, diminuisce gradualmente andando dal fondo-valle verso quote più elevate ".

Tengo qui a sottolineare che, l'estrema semplicità del metodo applicato, e la chiarezza delle registrazioni avute, danno ai risultati allora ottenuti un margine di certezza veramente inconfutabile, anche dal punto di vista qualitativo.

Lo spessore dellic parte sovrastante, di indubbio carattere franoso, fu determinato applicando metodi ed accorgimenti diversi, dei quali si è fatto cenno nella relazione alla SADE. L'uso di chiare riflessioni riscontrate nelle registrazioni ottenute, l'elaborazione di onde rifratte in corrispondenza del massiccio sottostante e di onde di esclusiva provenienza dallo strato franoso, nonché la registrazione di ampie onde di flessione, proprie dello strato stesso, ci hanno consentito di ottenere - per vie diverse - diversi valori dello spessore predetto, in corrispondenza dei tragitti sperimentati. Complessivamente, gli spessori ottenuti variano da un minimo di 10 metri, nei pressi della zona strapiombante, ad un massimo di 20 metri circa, nella parte centrale del rilievo eseguito.

Ciò ci permetteva di concludere che, "almeno per quanto riguarda $i$ profili lungo $i$ quali si è sperimentato, possiamo ritenere la zona in esame come costituita da un potente supporto roccioso autoctono, sul quale, attraverso $i$ tempi, si sono venuti a depositare materiali di frana, raccolti, come abbiamo detto, in una coltre di spessore variabile fra $i$ 10 e $i 20$ metri".

Era mio convincimento che i sistemi rocciosi di superficie, per quanto compatti, avessero risentito gli effetti - quali più, quali meno - delle potenti tensioni con le quali l'orogenesi aveva agito in essi, attraverso i millenni. Il che spiegava perché tutti apparissero più o meno profondamente crepacciati su diverse - per positura ed orientamento-superficie di frattura; e rendeva anche naturali certi echi da diverse provenienze, provocati da tratti di superficie riflettenti. Ma, come ebbi occasione di comunicare alla $S A D E$, non mi risultava che tali echi (pochi in realtà) si distribuissero sopra una unica superficie, almeno lungo i profili da noi 
indagati. Confermai che se era vero - come ritenevo fermamente - che il massiccio si era formato in loco, l'esistenza di una tale unica superficie era alquanto problematica.

\section{7. - Una terna di fotosismografi ad amplificazione elettromagnetica inizia il suo funzionamento presso la cabina comandi della diga.}

Come è stat to detto al termine del n. 5, verso la fine del 1959 una stazione sismica di notevole sensibilità, particolarmente studiata per il rilevamento delle scosse (microscosse) di carattere locale, iniziava il suo funzionamento (Fig. 13). Come in tutte le attività del genere, dapprincipio
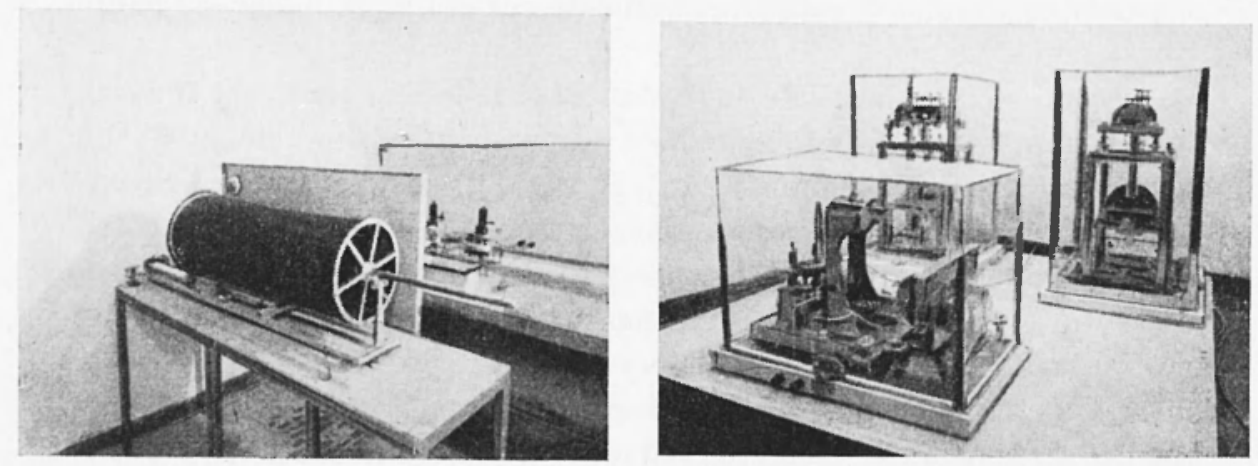

Fig. 13 - Prima stazione sismica presso la diga del Vajont.

il funzionamento presentò qualche - del resto inevitabile e più che giustificata-lacuna. Basti osservare che, stazioni di quel tipo, d'abitudine operano presso Istituti Universitari, dotati di particolari attrezzature e valendosi dell'opera di personale specializzato. Nulla di tutto questo, naturalmente, nell'impervia gola del Vajont, dove il personale addetto lavoravaa questo riguardo - in condizioni difficili. Se la stazione sismica entrò rapidamente in un assetto di sistematico funzionamento, si deve sopra tutto all'abnegazione, alla passione, all'interessamento amorevole e continuativo dei compianti fratelli Giovanni e Giorgio De Vido, alla memoria dei quali sovente va il mio pensiero ammirato e commosso.

Di questa stazione sismica è stato da me brevemente riferito in precedenti pubblicazioni.

Nei primi mesi del 1960, non si ebbero registrazioni appariscenti di carattere locale. A proposito di tali registrazioni, non è forse superflua una 
precisazione. Chi ha qualche nozione di sismologia, sa che l'interpretazione di tali scosse non rientra nella metodologia ordinaria, sostanzialmente basata sull'uso di tabelle già calcolate e universalmente diffuse, e potendo
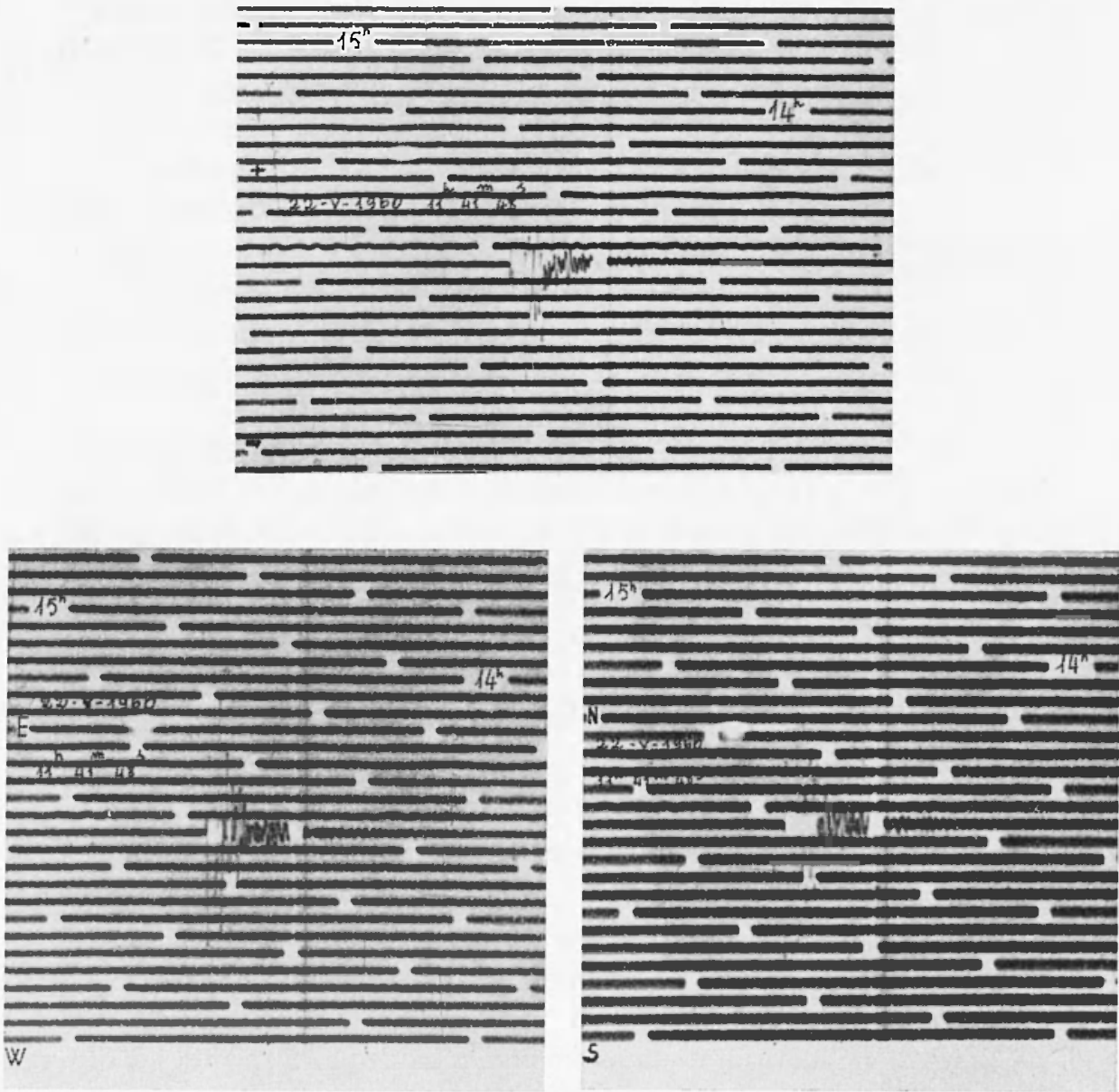

Fig. 14 - Prima sensibile microscossa registrata dalla stazione sismica del Vajont, sistemata in cabina comandi.

usufruire di un certo numero di fasi, scaglionate nel tempo, già ben classificate, come appunto avviene per terremoti, provenienti da distanze di almeno qualche diecina di chilometri. Nulla di tutto questo, nel caso in esame. L'analisi di microseosse-provenienti da qualche centinaio di metri di distanza_ richiede conoseenze specifiche sulla natura del suolo, in cui le scosse stesse si verificano, un bagaglio teorico non comune e - se è 
consentito - sopratutto un " quid " che la scuola non dà, che non possono dare i libri e che solo scaturisce da un'esperienza, che non esito a definire "sofferta ", conseguita con tenacia, non disgriunta da umiltà e da un senso di amore per le cose. Questa esperienza avevo avuto modo di conseguire negli estenuanti larori d'interpretazione delle centinaia e centinaia di piccole scosse, registrate nella stazione sismica funzionante presso la diga di Pieve di Cadore, specie nei primi anni (dal 1950 al 1953). Fu quella la prima esperienza del genere che veniva effettuata nel mondo; e gli insegnamenti che ne trassi mi riuscirono preziosissimi nella nuova, più impegnativa fatica. L'abito elastico della zona era già stato determinato con precedenti indagini; l'acquisizione di queste conoscenze, unita alla somma delle cognizioni particolarissime sopra cennate, permise di trasferire, alla zona del Vajont, lo strumento interpretativo, approntato a Pieve di Cadore.

La prima scossa locale, di apprezzabile intensità, fu quella registrata il 22 Maggio 1960 (Fig. 14). Essa proveniva da ma distanza di $2 \mathrm{~km}$ ea, secondo un azimut di $100^{\circ}$ ca. L'epicentro, pertanto, risultava alla base del Toc sulla sinistra del Vajont.

\section{8. - Indagine geosismica condolta nel mese di Dicembre 1960 a monte della diga del Vajont, in sponda sinistra.}

Il verificarsi di una numerosa serie di microscosse locali, avvenute nell'Autunno del 1960 (e di cui diremo più avanti), aveva suggerito l'opportunità di ripetere, ampliandole, le indagini geosismiche eseguite nel Dicembre 1959.

Oltre che con vibrometri, le esperienze del Dicembre 1960, furono completate con registrazioni di un apparecchio per prospezione sismica a 12 tracce (geofoni).

La relazione sui risultati di questa nuova indagine fu spedita all'Ufficio Studi della SADE in data 10 Febbraio 1961.

La situazione nella zona era mutata. Lo sperone di notevole rigidità, investigato l'anno precedente, aveva subito nel frattempo un'azione di frantumamento, che ne aveva attenuato la risposta elastica. Anche al di fuori di detto sperone, lungo le sezioni trasversali alla valle, la situazione era quella di un mezzo incoerente, fino a notevoli profondità, che - dalla superficie esterna - superavano in qualche punto il centinaio di metri.

"Al di sopra delle profondità accennate (c̀ detto nella prima parte della citata relazione), non è il caso di parlare di vere e proprie superficie di discontinuità. Sostanzialmente le velocità dalla superficie esterna alla 
più volte accennata discontinuità profonda, vanno crescendo da $\approx 00$ misec circa ai $2500 \mathrm{~m} / \mathrm{sec}$, con salti piuttosto disordinati, caratteristici $\mathrm{di} \mathrm{um}$ mezzo fortemente inomogeneo, come conseguenza di un deterioramento che rimane evidente fino a notevole profondità. Solo in corrispondenza della parte più alta della sezione, le velocità che si osservano a profondita fra $i 40 e$ i 50 metri (dell'ordine di $3,5 \mathrm{~km} / \mathrm{sec}$ ) sembrano proprie di un meza compatto. Ta notato a questo riguardo che dette velocità sono dell'ordine a $i$ quelle a suo tempo registrate nella parte piu alta della zona, alla quale la diga è ancorata ".

Ma ormai non vale soffermarsi sui particolari di quella campagna geosismica. I delineare Io stato del campo elastico alla fine del 1960, mi sembra più appropriato continuare a riportare parte della relazione 10 Febblaio 1961, più volte citata, che ad esso si riferisce.

"Indipendentemente dai risultati ottenuti e più sopra riportati, che vanno considerati di prima 'grossolana' approssimazione, attesa la complessità della zona, riveste a nostro avviso, notevole importanza il confronto delle conclusioni ottenute a seguito delle indagini del Dicembre 1959 ( $\mathrm{T}$. relazione del 4.II.1960), condotte lungo una sezione che, partendo dal greto del fiume, a quota 535, terminava a quota 850 , nei pressi della nuova strada in sinistra (disegno no $482 \%$ del 10.XII.19.59), con le interpretazioni delle registrazioni ottenute lungo la prima sezione del Dicembre 1960, nei tratti in cui le sezioni si intersecano.

Il contrasto è veramente nettissimo. Ste, in un primo tempo, limitiamo il confronto alle velocità medie osservate, notiamo che ai 5 -6 $\mathrm{km} / \mathrm{sec}$ del $1 \mathrm{Di}$ cembre 1959, fanno riscontro $i 2,5-3 \mathrm{~km} / \mathrm{sec}$ ottenuti nel Dicembre 1960 nella stessa zona, come velocità massima. Nel periodo appena di un anno si è verificata quindi, nel tratto in parola, una cospicua diminuzione di velocità, come indubbia conseguenza di un'azione di frantumamento del setto roccioso, di roccia robustissima, che reggeva - almeno fino al Dicembre 1959_il sovrastante carico di materiale di frana. ì confermato infatti che, sotto l'azione di pressioni crescenti, un mezzo roccioso, anche se robustissimo, finisce col cedere gradualmente, fiaccato nella resistenza del suo tessuto molecolare $\left.{ }^{3}\right)$. Dapprima la velocità delle onde longitudinali tende a crescere con la pressione, fino al punto in cui - per ulteriore aumento di pressione la velocità tende a diminuire, dapprima lentamente, quindi sompre piu rapidamente, per ridursi a valori assai bassi in fase di rottura del mezzo resistente.

L'andumento del fenomeno è sintetizzato nel diagramma in Fig. 1 (ora Fig. 15); come si vede, in esso si distinguono tre fasi nel materiale sottoposto 
a pressione idrostatica crescente: fase elastica, con velocità di propagazione in lieve aumento, fase plastica, con velocita di propagazione in lieve diminuzione, e fase di rottura, in cui la velocità descresce rapidamente. La Fig. 2 (qui non riportata) fornisce il diagramma relativo al caso particolare dell'esperienza su un campione di marmo (pag. $\$ 11$ - E.R.I. $X X X I I I-3-1955)$.

Tale fenomeno è intervenuto nella roccia sottostante la zona in questione, roccia che nel Dicembre 1959 iniziava alla profondità media di 20 metri circa e che attualmente ivi si trova, fiaccata nelle sue caratteristiche clastiche. In detta zona, riflessioni apprezzabili vengono infatti da profondita medie dell'ordine di 100 metri, dove il mezzo conserva ancora notevole il suo coefficiente di forma.

L'aumento di pressione che ha determinato il decadimento della roccia nel luogo suddetto, può essere stato provocato dal cedimento dei diaframmi di roccia compatta, posti a quota maggiore. Ciò in conseguenza delle numerose scosse sismiche verificatesi durante $i$ primi mesi del 1960 e fino a metà Novembre dello stesso anno.

Quanto è stato sopra detto viene confermato dalla frequenza delle vibrazioni longitudinali, registrate nella zona in esame, e nel Dicembre 1959 e nel Dicembre 1960. Mentre nel 1959 tali frequenze furono molto elevate (corrispondendo a periodi di 0,005 sec) nel Dicembre 1960 esse erano sensibilmente diminuite (corrispondendo in media a periodi di 0.02 sec) [Fig. 16].

$\dot{E}$ stato dimostrato che la rigidità del mezzo è associata a frequenze tanto più elevate quanto più grande è il coefficiente di forma. La notevole diminuzione della frequenza riscontrata nella zona sopra detta, testimonia pertanto del decadimento delle sue caratteristiche elastiche".

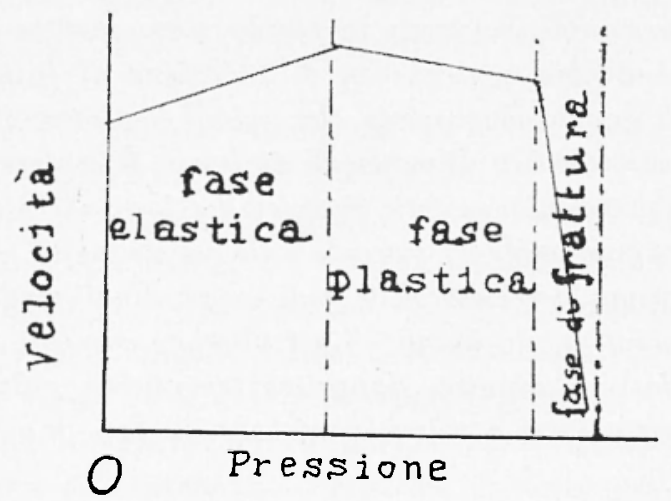

Fig. 15 - Alterazione del campo elastico in un mezzo solido, sotto l'azione di pressioni crescenti (secondo Shimozuru). 

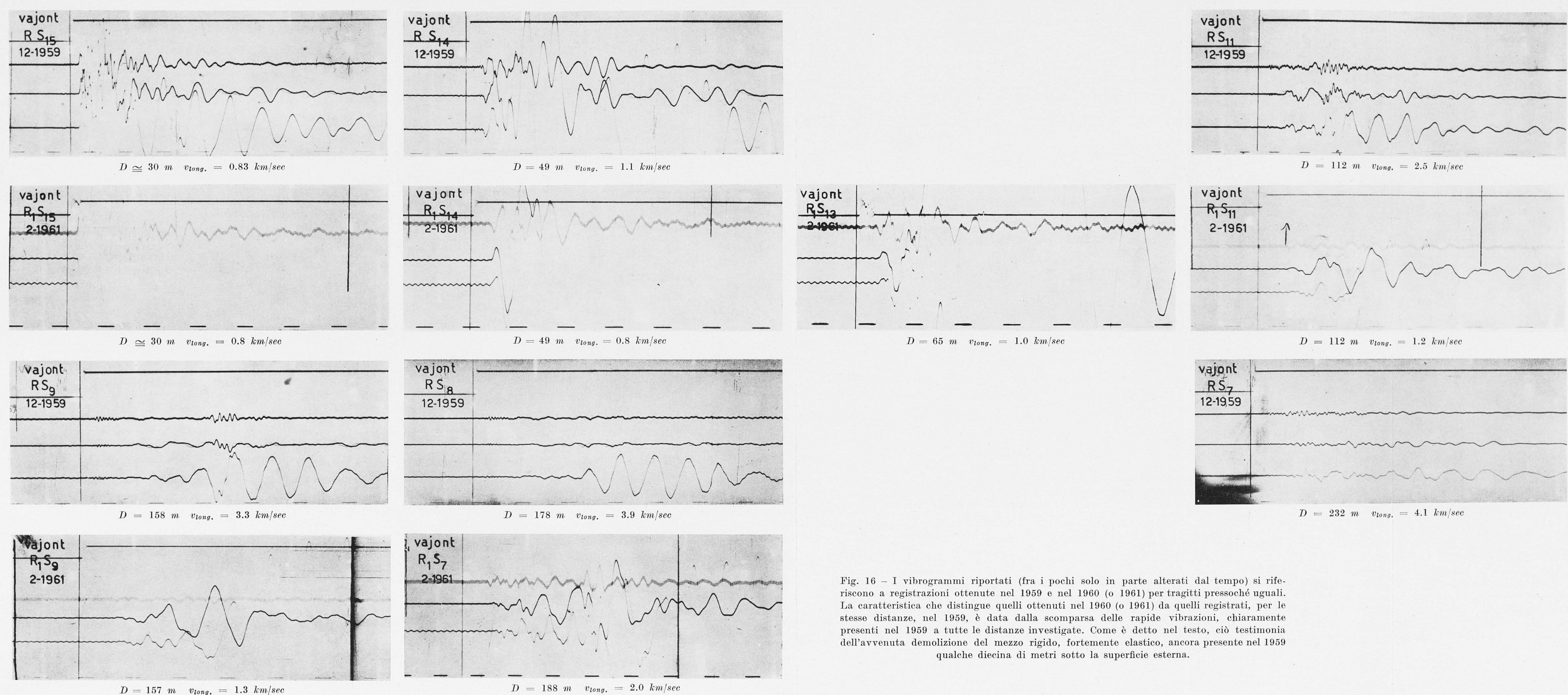

Fig. 16 - I vibrogrammi riportati (fra i pochi solo in parte alterati dal tempo) si rife-
riscono a registrazioni ottenute nel 1959 e nel 1960 (o 1961) per tragitti pressoché uguali. La caratteristica che distingue quelli ottenuti nel 1960 (o 1961 ) da quelli registrati, per le

peesenti nel 1959 a tutte le distanze investigate. Come è detto nel testo, ciò testimonia
dell'avvenuta demolizione del mezzo nividio, fortemente elastico, ancora presente nel 1959 
Prescindendo dai valori del modulo di elasticità, mi permetto di sottolineare due fatti (del resto richiamati nella relazione) a testimonianza della modificazione, subita dal mezzo investigato nel Dicembre 1959: la diminuzione della velocità delle onde longitudinali e la scomparsa delle vibrazioni di elevata frequenza. Il primo fatto è sintetizzato nella Fig. 17, dove vengono messe a confronto le velocità osservate nel 1959 e nel 1960, sulla stessa distanza. Il diagramma è tale da escludere ogni possibilità di obiezione. Ma non meno innegabile è la scomparsa delle iniziali altissime frequenze.

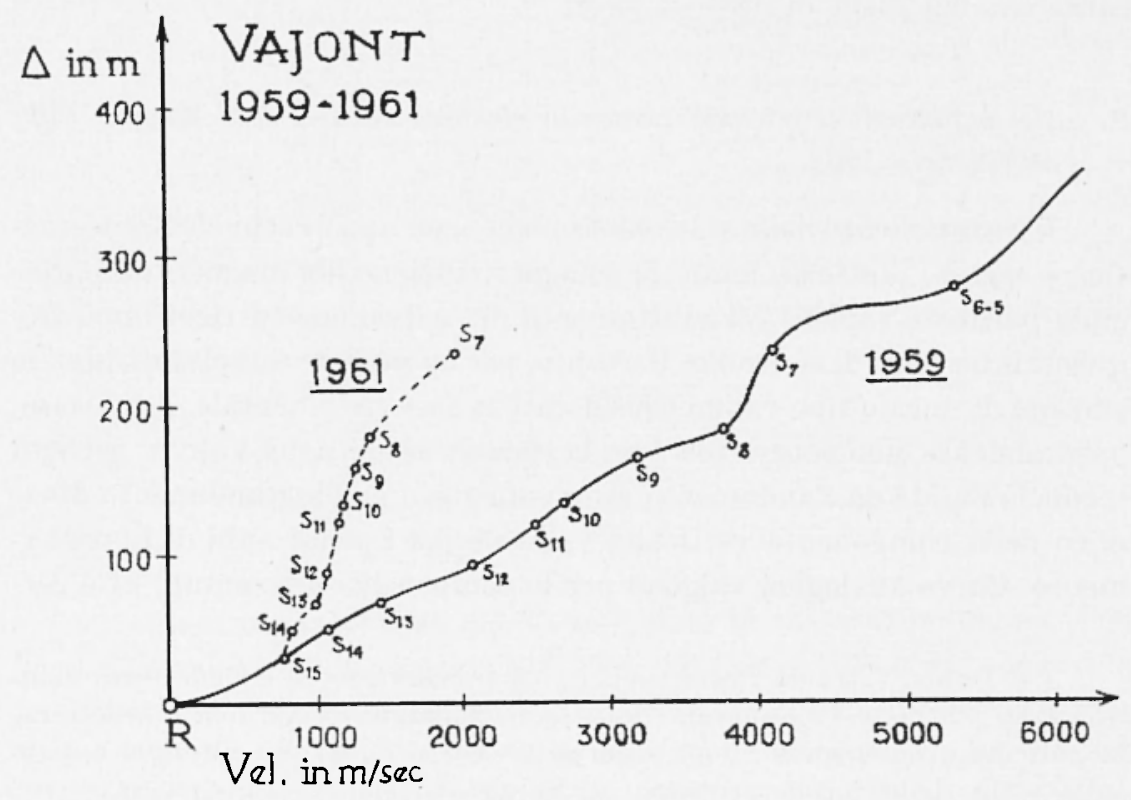

Fig. 17 - Andamento della velocità apparente delle onde longitudinali, con la distanza, su tragitti di analoghe caratteristiche, quale risulta dalle indagini del 1959 e del 1961. Le osservazioni del 1960-61 attestano l'indebolimento in campo elastico del mezzo rigido, sottostante la coltre alluvionale di 20-30 metri di spessore, ancora presente nel 1959. Tale indebolimento è attestato altresì dalla scomparsa delle onde longitudinali di elevata

frequenza, proprie di quel mezzo, precedentemente molto compatto.

Ho già provato come le elevate velocità siano associate alle massime frequenze, tantoda dar luogo al fenomeno della dispersione anomala. Ho avuto modo di dimostrare la reale esistenza di detto fenomeno in centinaia di esperienze. Pertanto, la scomparsa delle elevatissime frequenze iniziali, sta a dimostrare che il mezzo, che nel 1959 ancora le 
conduceva, nel Dicembre 1960 non era più nelle condizioni di farlo. Questo discorso si traduce, in campo físico, dicendo che il mezzo aveva perduto la rigidità primitiva, ciò che si spiega appunto con una diffusa fratturazione.

Come si è griunti a questa rapida modificazione del tessuto elastico? La causa più plausibile è quella riportata nella relazione: le numerose scosse sismiche locali, verificatesi come vedremo, proprio alla base della zona in questione: ne."Autunno del 1960, hanno indubbiamente contribuito alla progressiva frantumazione dello sperone roccioso, in coneomitanza con fenomeni di "cretping" (*).

\section{9. - Le registrazioni ottenute presso la stazione sismica dal Maggio 1960 all'Ottobre 1963.}

L'assestamento delle stazioni sismiche, con apparechi elettromagnetici, è sempre piuttosto lento: la smagnetizzazione dei magneti (dapprincipio piuttosto rapida) e l'adattamento dei galvanometri richiedono frequenti interventi di controllo. Pertanto, per un periodo talvolta di anni, le stazioni di questo tipo vanno considerate in fase sperimentale. $A$ tale fase, naturalmente, non poteva sottrarsi la stazione sismica del Vajont. Ad ogni modo, la Fig. 18 dà l'andamento approssimativo dell'ingrandimento dinamico della componente verticale, valevole per i primi anni di funzionamento. Curve analoghe, valgono per le componenti orizzontali. Si è cer-

(*) Debbo tuttavia ricordare che, nel trasmettere all' Ufficio studi della SADE la relazione 10 Febbraio 1961 , la accompagnavo con una mia lettera, in pari data, nella quale, dopo essermi riservato di fornire ulteriori notizie sulla stato della roceia prossima al Vajont, in sponda sinistra, scrivevo: "Aggiungo - a mo' di corollario di quanto è detto nella relazione -.. che da oltre due mesi non si hanno al Vajont registrazioni di scosse locali. 1 mio avviso, ciò è di buon augurio: sembra quasi che l'acme della erisi sia passata".

Poco dopo scrivevo ancora che il fenomeno di fratturazione della roecia sembrava andare attenuandosi con la profonclità. sicché era da ritenere che sul greto del fiume la roccia non fosse che in parte fiaccata nella sua resistenza.

"Sembra - serivevo altresì il 2.IJI.1961 all' Officio Studi della SADE -che la roccia di fondo sia ancora abbastanza consistente".

Ogri, dopo l'evento del 9 Ottobre 1963 , mi incombe il dovere di precisare che quanto esposto nella mia relazione del 10.1 I.1961 ed in quella del 10.IV.1962, nonché quanto rilevato, anche successivamente, durante il corso dei miei studi nella zona - prima della frana dellottobre 1963 - non mi indusse mai ad immaginare possibile uno scoscendimento immane avente le caratteristiche - senza precelenti, per quanto mi consta - di quello verificatosi. 
cato di tenere le tre componenti - e, in modo speciale, le componenti orizzontali - con ingrandimenti approssimativamente uguali, così da poter determinare gli azimut (e, suceessivamente, gli angoli d'emergenza apparenti) senza la necessità di calcolare gli spostamenti reali del suolo.

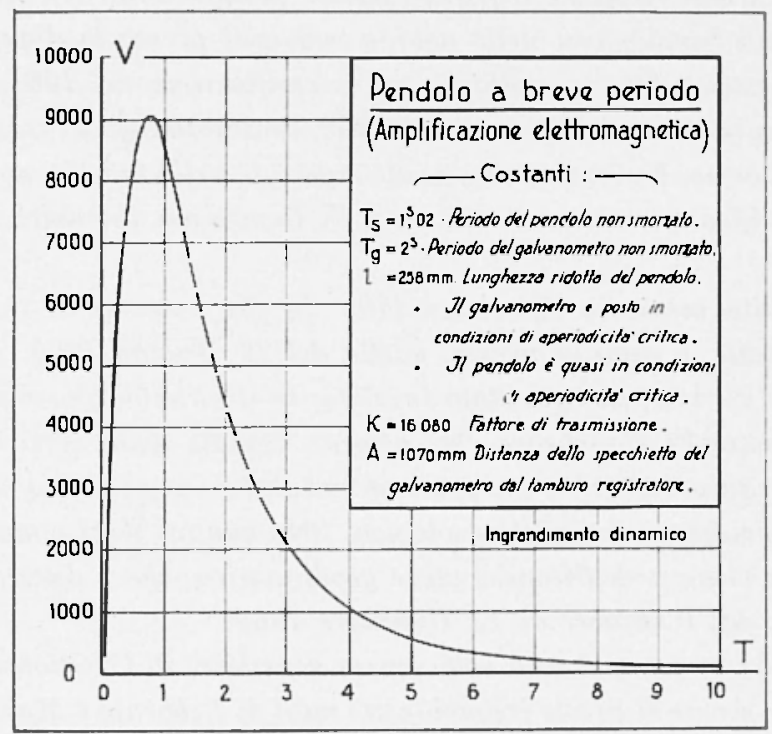

Fig. 18

La stazione sismica del Vajont, data la sua sensibilità, la oftenuto tutta una serie di registrazioni, che esula da quelle strettamente locali, e che presentano invece notevole interesse in ricerche di carattere generale.

Di esse, naturalmente, qui noi non ci occuperemo, riservando la. nostra attenzione a quelle originate nella valle del Vajont o in zone confinanti. Nei loro riguardi, inizanlmente il mio impegno fu esclusivo; successivamente, ebbi ad usufruire dell'efficace collaborazione della Dott.ssa M. C. Spadea.

Anziché riesporre i risultati delle interpretazioni sismiche, ottenute in occasione di microscosse locali, ritengo fic opportuno riportare le relazioni, di volta in volta inviate alla S.IDE sull'argomento: ciò allo scopo di conservare quel carattere di immediatezza e spontaneità, che generalmente contraddistingue le prime interpretazioni.

Il 10 Aprile 1962, nel presentare la Tabella I, a due grafici (che qui non riporto, perchè assorbiti in quelli suceessivamente aggiornati), così mi esprimevo: 
" Un breve commento alla tabella e ai relativi grafici allegati.

Nella tabella sono riportati i tempi di inizio, l'azimut di provenienza (contato da Nord, in senso orario), la distanza d'origine, il senso di spostamento dell'inizio (onde longitudinali) e lo spostamento massimo del suolo, relativi a 43 scosse sismiche registrate dal 22 Waggio 1960 al $1^{\circ}$ Aprile 1962, dai sismografi funzionanti nella cabina comandi presso la diga del Tajont (sponda sinistra). Di esse soltanto tre si verificarono nel 1961: delle altre, 28 furono registrate nel 1960 e 12 nel 1962, limitatamente ai trascorsi primi tre mesi dell'amo. Naturalmente, quelle riportate nella tabella, non sono tutte le scosse verificatesi; un certo numero, di lievissima intensità, non figura nell'elenco.

Ta subito messo in rilievo un fatto: la piu intensa di tutte le scosse finora registrate, ì stata la prima, quella del 22 Maggio 1960. Non so se a quell'epoca l'invaso fosse già stato iniziato: se il serbatoio fosse stato ancora vuoto, ciò potrebbo significare che, almeno l'inizio della crisi sismica, ha avuto come causa esclusiva un processo naturale, in una zona - per molti segni - di evidente instabilità geologica. Solo quattro mesi e mezzo dopo, si è avuta una ripresa dell'inquietudine geodinamica, che è durata, pressochè ininterrotta, dal 6 Ottobre al 13 Dicembre 1960.

$N^{+}$el 1961 si è avuta una sola scossa sensibile, il 1\% Ottobre; mentre il numero delle scosse si è fatto frequen te nei mesi di Febbraio e Marzo dell'anno in corso. Nel complesso però, almeno finora (10 Aprile 1962), l'intensità delle scosse è inferiore a quella registrata nel periodo di forte attività alel 1960.

A mio pareve, un'importante deduzione può essere tratta dall'esame della natura dell'onda longitudinale, quale risulta dall'inizio della registrazione dello scosse registrate. Ira ricordato che l'onda longitudinale provoca lo spostamento di una particella raggiunta dal movimento nella direzione del moto stesso: essa determina un moto di dilatazione, quando il verso del moto è quello che va dalla stazione di registrazione all'ipocentro; determina, invece, una compressione in caso contrario. In altri termini, in corrispondenza di una dilatazione si verifica un richiamo di terreno verso l'ipocentro, mentre una compressione qualifiea un movimento dall'ipocentro alla stazione d'osservazione: nella situazione in esame, al primo caso corrisponde un avvallamento del suolo all'ipocentro, al secondo una spinta verso la superficie esterna. A questo proposito, è interessante osservare che $i$ movimenti iniziali di dilatazione hanno nettamente prevalso su quelli di compressione: 24 contro 14, fra le scosse riportate.

I a inoltre sottolineato il fatto che la totalità delle dilatazioni (con due sole eccezioni) proviene da un ristretto settore, compreso fra $i 100^{\circ}$ e $i 114^{\circ}$ 

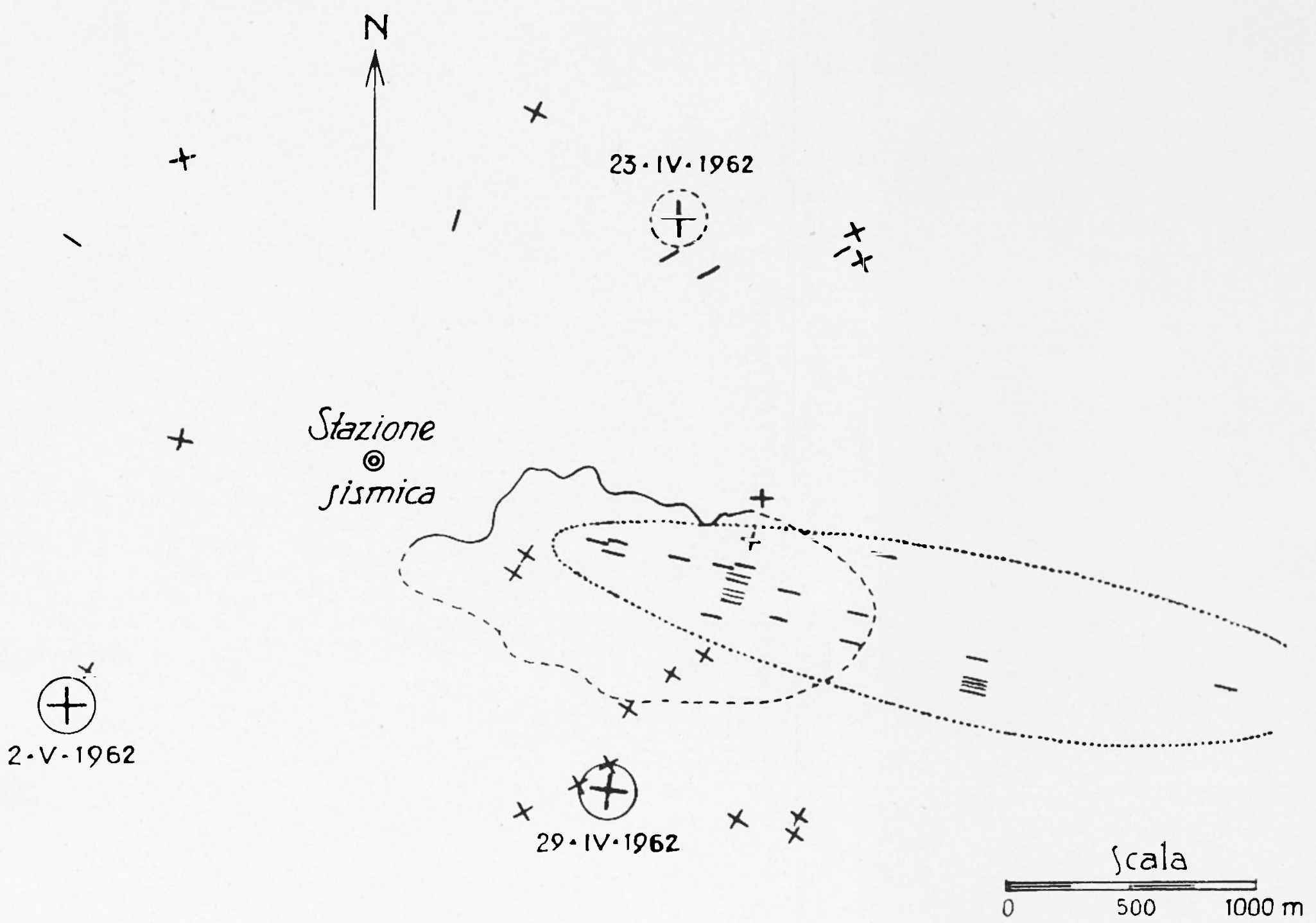

Fig. 19 - Epicentri delle principalii microseosse osservate presso la stazione sismica del Vajont dall'inizio del funzionamento della stazione sismica ai primi di Maggio 1962. 
$x$

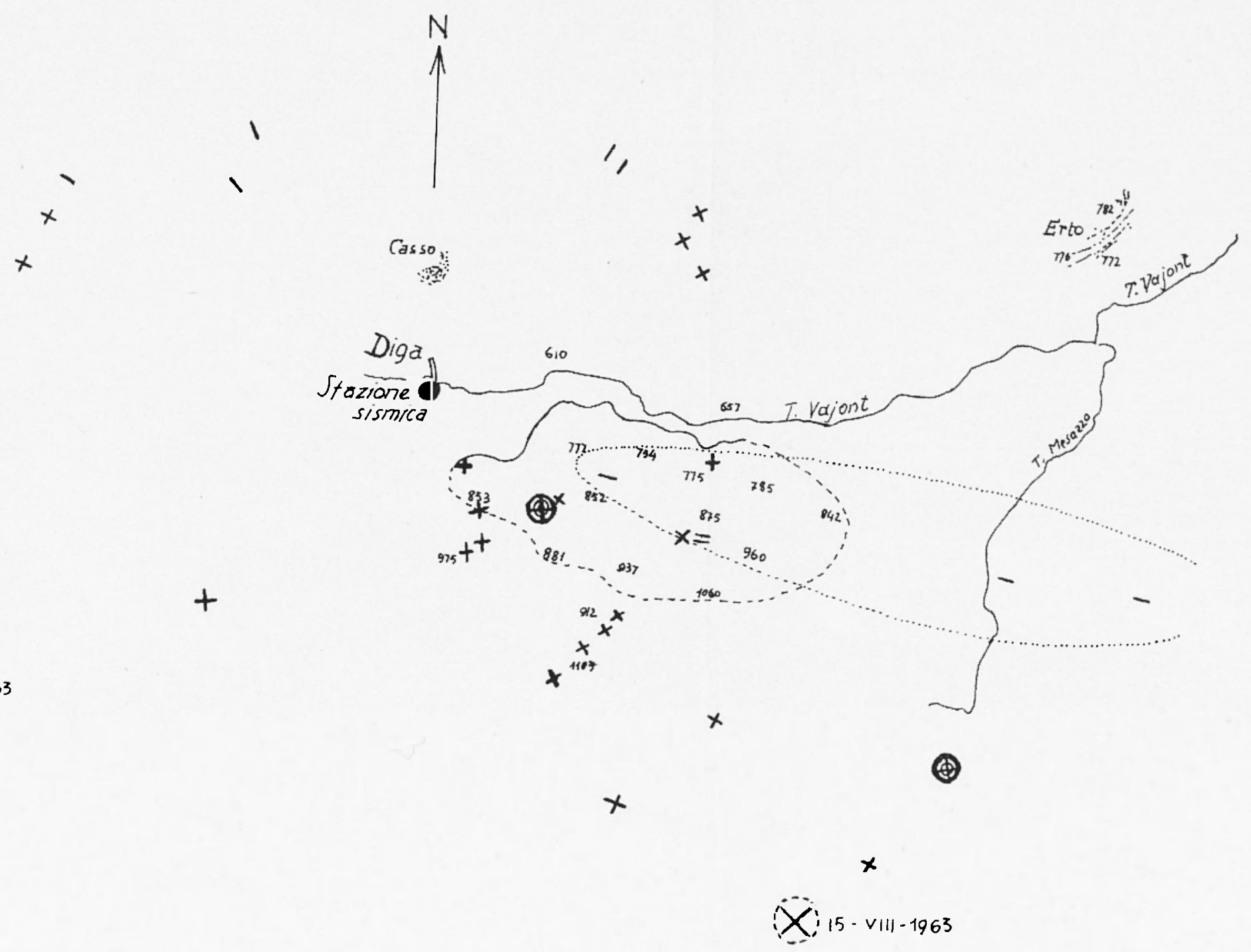

Fig. 20 - Epicentri delle principali microscosse registrate presso la stazione sismica del Vajont dal 15 (iiugno 1962 al 15 Agosto 1963. Risulta evidente che l'ultima fase dell'attivita microsismica - che ha preceduto l'evento del Vajont - è stata caratterizzata dalla netta prevalenza delle compressioni $(+)$. lungo le pendici del Toc. 


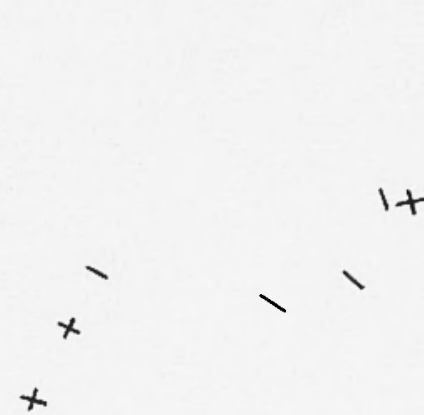

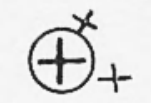

$2 \cdot V \cdot 1962$

(d) $15 \cdot V 111 \cdot 1963$

Scala

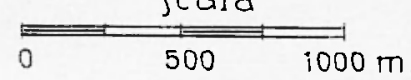

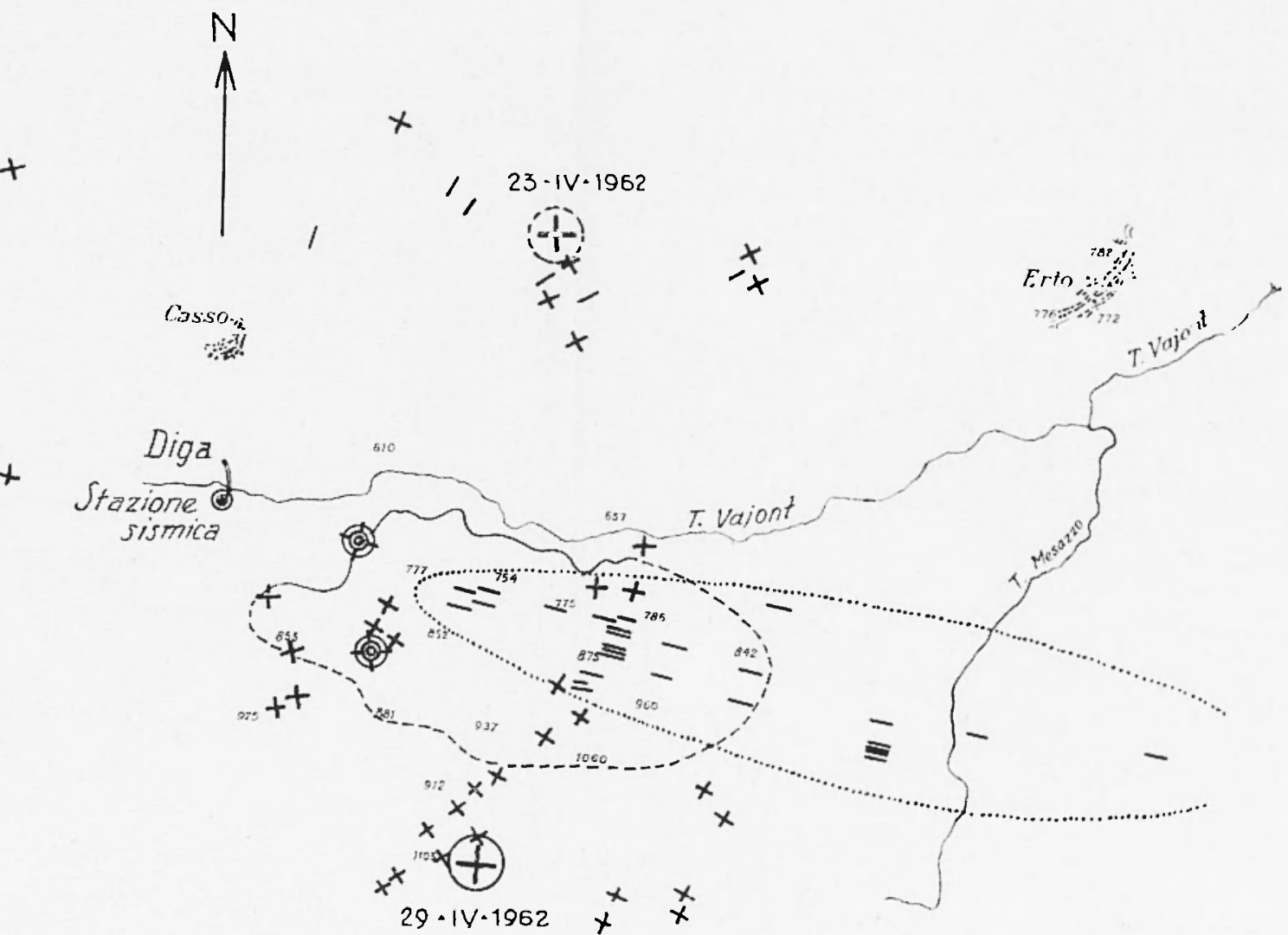

(8)

X; $15 \cdot v 111 \cdot 1963$

Fig. 21 - Planimetria complessiva delle principali microscosse registrate al Vajont - a tutto Settembre 1963 - dalla prima stazione sismica. Il segno - significa moto verso l'interno (dilatazione), il segno + verso l'esterno (compressione). 

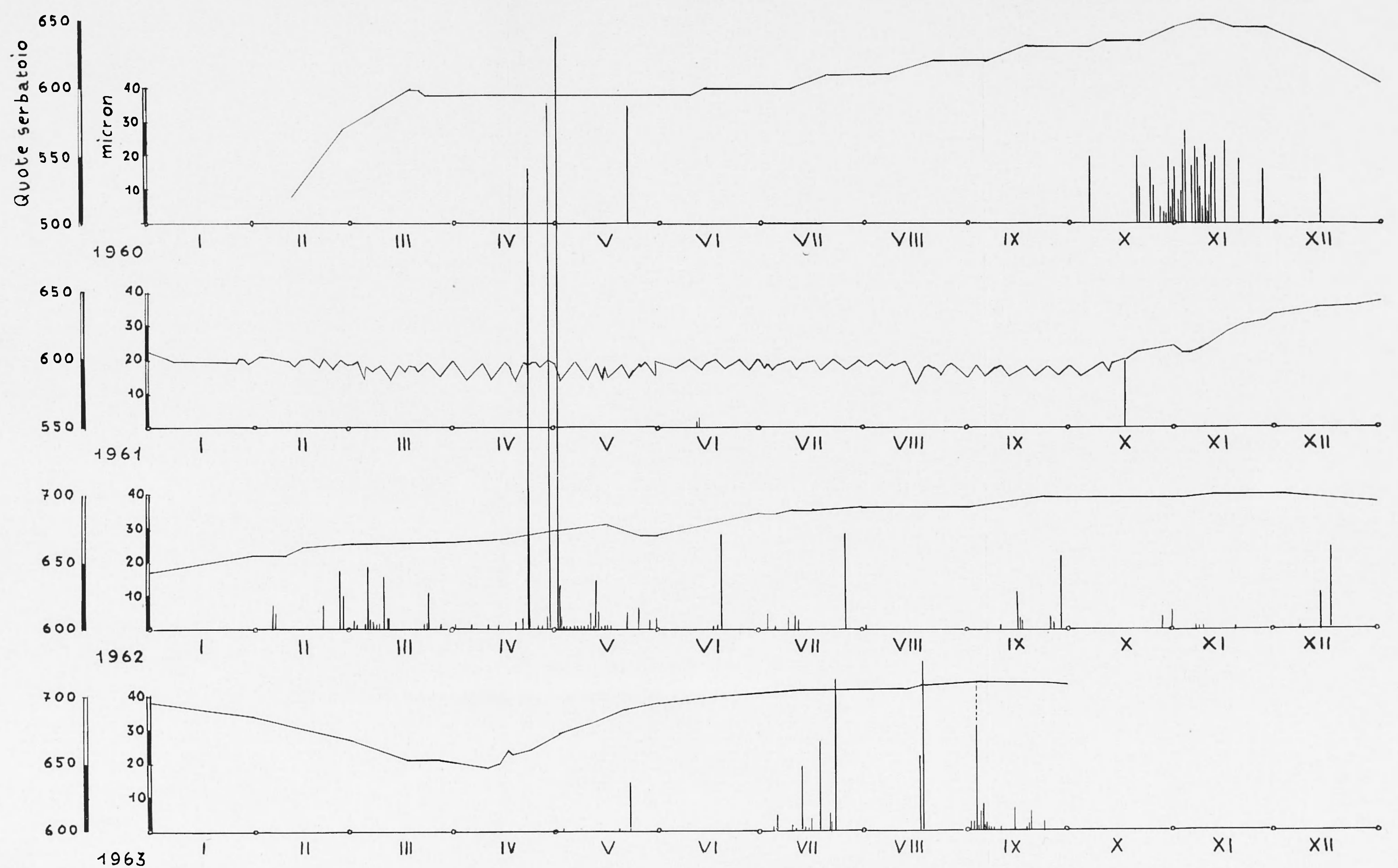

Fig. 22 - Variazione del livello dell'acqua nel serbatoio del Vajont, e ampiezze massime approssimative in mieron delle principali microscosse registrate nel periodo Maggio 1960-Settembre 1963. 
di azimut: su 23 scosse, 18 con variazione massima di azimut di soli 5o e 16 su 18 addirittura dalla stessa divezione (intorno a 110 di azimut). Ciò sta a provare che l'origine delle dilatazioni è unica: se poi si osserva, tenendo conto delle distanze rilevate, che tutte le dilatazioni cadono nella zona di frana, si può senz'altro attribuire le scosse relative ad un fenomeno di abbassamento, in atto nella zona in questione. Le compressioni invece abbracciano una zona molto più estesa, quasi eschusivamente nel settore di SE, verso la zona montuosa in sponda sinistra. Un'interpretazione geodinamica potrebbe essere la seguente: all'abbassamento in atto in corrispondenza della frana, fa riscontro un fenomeno di lievissimo sollevamento nei fianchi montuosi che si dipartono a ventaglio dal luogo stesso. Sembra quasi che un fenomeno contrastante caratterizzi il movimento della zona, come un persistente residuo di locale orogenesi. Va infine osservato che l'inquietudine in atto in questi ultimi mesi ̀̀ soprattuto contrassegnata du compressioni, come se in questo periodo prevalesse la spinta verso l'esterno.

Per quanto concerne l'attività sismica nella regione circostante, essa è stata limitatissima in tutto il periodo in esame. Una sola scossa infatti, si è verificata nel raggio di $20 \mathrm{~lm}$ dal Vajont; quella registrata il $1 \mathrm{r}$ Marzo 1962. Il suo epicentro è stato determinato sulla base delle chiare registrazioni ottenute a Pieve di Cadore, Vajont e Tolmezzo. La distanza epicentrale da queste tre stazioni sismiche è risultata di 12,6 lim, 16,8 $\mathrm{k}: \mathrm{m}$ e 38,5 lim rispettivamente. Ciò ha permesso di localizzare l'epicentro nella zona di Pramaggiore (monte), a NE ali Cimolais ».

La seconda relazione alla SADE è del 15 Giugno 1962. Eccola:

"Nella precedente relazione era stato riferito sulla sismicità in atto nella valle del Tajont fino al 10 Aprile c.a. Diremo ora brevemente sulle scosse verificatesi oltre tale data.

L'attività è ripresa in modo piuttosto accentuato nella terza decade di Aprile, protraendosi, con alterna frequenza ed intensita, fino ai primi ai Giugno (precisamente fino al 10 Giugno, data in corrispondenza della quale si chiude per ora la nostra indagine). La Tabella con l'elenco delle scosse registrate in questo periodo - tralasciando quelle di trascurabile intensità è riportata in fondo alla relazione.

Le manifestazioni più notevoli sono quelle verificatesi il 23 e 29 Aprile e il 2 Maggio (Fig. 19). Hanno avuto intensità pressoché uguale, leggermente crescente dalla prima alla terza: sono comunque le tre scosse piu notevoli registrate in questi due ultimi anni. Di esse hanno fornito chiare registrazioni anche lo stazioni sismiche di Pieve di Cadore (alla distanza di $7 \%$ lim ca) e di Tolmezzo (alla distanza di $5.5 \mathrm{him} \mathrm{ca}$ ). 
Nella breve relazione precedente, veniva messo in risalto il fatto che contrariamente a quanto si era verificato nel recente passato - l'inquietudine in atto è soprattutto contrassegnata da compressioni, come se in questo periodo prevalesse la spinta verso l'esterno, quale conseguenza di un residuo di orogenesi locale.

I. fenomeni degli ultimi due mesi sembrano confermare questa conchsione: le compressioni infatti hanno avuto netta prevalenza, provenendo dalle direzioni piu diverse; ad ogni modo, esternamente alla zona a monte della diga, caratterizzata dal fenomeno contrario (frana). Ciò è sintetizzato nella Fig. 2 (ora integrata nella Figr. 20).

Abbiamo già osservato che la zona in questione è stata caratterizzata anche in passato da una sismicità piuttosto sostenuta. Va ricordato p.e. che il 27 Dicembre 1933 nei dintormi di Longarone si verificò una scossa sismica registrata in un raggio di cirea j00 lim. Da uno studio eseguito nel $\left.1934{ }^{4}\right)$, l'epicentro di tale scossa risultò nel punto di coordinate geografiche

$$
\begin{array}{ccc}
46^{\circ} & 15^{\prime}, 7 & N \\
12^{\circ} & 19^{\prime}, 4 & E,
\end{array}
$$

punto che coincide praticamente con la zona della diga del Vajont.

Le scosse dell'attuale periodo sono di una intensità molto minore di quella ora richiamata, pur rientrando evidentemente nello stesso ordine di cause. Va ancora sottolineato il fatto che la sismicita di cui soffre attualmente la valle del Tajont rientra in un'inquietudine più generale, che attualmente sta interessando le Prealpi Bellunesi. A testimonianza di quanto affermiamo stanno le diecine e diecine di scosse strumentali che vengono registrate, nel periodo di cui ci stiamo interessando, dalla stazione sismica di Pieve di Cadore; scosse che hamno una provenienza del tutto diversa da quella della valle del Vajont. Ciò è documentato nella Tabella II (ora I.).

Con quanto precede non si intende cschudere la possibilita di un intervento perturbatore da parte del bacino idrico. Il lieve incremento di pressione derivante dall'invaso e la penetrazione delle acque nelle stratificazioni superficiali, possono agive da cause concomitanti, in una zona caratterizzata da naturale sismicità.

L'accumulo delle tensioni derivanti dall'orogenesi residua può essere disturbato dalla leggera variazione di pressione sopra accennata, e questo aisturbo può tradursi in una serie di piccole fratture anticipate - di natura prettamente superficiale - su quella che dovà essere la fase di rottura legata al ginoco delle forze naturali (generalmente verificantesi a profondita molto piu accentuata). 
Concludendo, intendiamo dive che la molestia derivante dall'intervento dell'uomo, può provocare movimenti esclusivamente superficiali di limitata intensità; scosse più intense e profonde debbono ascriversi a cause naturali.

Ci riserviamo di seguire il fenomeno nei suoi ulteriori sviluppi. Per. ora, ad ogni modo, esso resta inquadrato come aspetto particolare di una inquietudine sismica che interessa una zona più estesa ".

In questa seconda relazione, viene posto l'accento sulla constatazione che, in un secondo tempo, sono le compressioni a prevalere nettamente sulle dilatazioni. Sembra quasi che il monte, liberato dai precedenti cedimenti sul fondo (dilatazioni) da vincoli che ne limitavano i movimenti, possa ora più liberamente manifestare la sua residua tendenza a salire: di qui il netto prevalere delle compressioni, tutte sparpagliate a Sud della fascia di cedimento, nell'interno della montagna.

Il 2 Settembre 1963 i sismografi delle stazioni presso le dighe del Vajont e Pieve di Cadore, registrarono una scossa con epicentro nella zona del Col Nudo. In seguito ad essa, come succede sovente in zone instabili, si verificò una lieve recrudescenza locale.

La terza relazione è del 5 ottobre 1963. Essa viene qui di seguito riportata.

1. - In questa terza relazione sull'attività sismica, a carattere locale, registrata dalla stazione sismica funzionante presso la diga del Tajont, si riportano $i$ dati essenziali relativi alle scosse che si sono susseguite dal Maggio 1962 al 30 Settembre 1963. Il fatto piu caratteristico di detta attività e. senza dubbio quello contrassegnato dal contrasto tettonico in atto in sponda sinistra del bacino, lungo le pendici del monte Toc. All'avvallamento corrispondente alla zona franosa, fa riscontro il sollevamento lungo il margine settentrionale del Toc; evidentemente, $i$ due movimenti sono legati come da causa al effetto. Il senso del moto iniziale delle registrazioni, a questo riguardo, ̀̀ quanto mai significativo; dilatazioni per le scosse originanti nel tratto franoso, sistematiche compressioni piu in quota, lungo i fianchi del Toc (Fig. 21).

L'attività sismica non si limita naturalmente a quella strettamente locale. Come si è già detto in precedenza, anche nelle zone limitrofe, di quando in quando, si verificano movimenti sismici, di piccola intensità; negli ultimi due anni, particolarmente frequenti quelli originanti alal Monte Pramaggiore e dal Col Tisentin. Abbiamo aggiunto un elenco di registrazioni, ottenute nello stesso periodo a Pieve di Cadore, stazione sismica piu prossima 
a H. Pramaggiore. Ciò a testimonianza di un'attività sismica per mulla legata alla creazione del bacino idrico del Vajont. A questo proposito, va sottolineato che la scossa piu sensibile, sentita da Longarone a Cimolais e da Castellavazzo a Soverzene, quella del 2 Settembre 1963, ha avuto origine dalle pendici del Col (Crep) Nudo, a circa $6 \mathrm{~km}$ dal Vajont. Le registrazioni ottenute presso quest' ultima località, a Pieve di Gadore (alla distanza di $17 \mathrm{~km}$ ), a Somplago (alla distanza di lim :j:,j) e a Tolmezzo (distante 50 km), hanno consentito la determinazione delle coordinate epicentrali e dell'ora origine del terremoto:

coordinate epicentrali $, \quad \varphi=46^{\circ} 14^{\prime}, 7 \mathrm{~N} ; \quad \lambda=122^{\circ} 26^{\prime}, 9 E$ (Col Nudo) ora origine: $10^{\mathrm{h}} 18^{\mathrm{m}} 40^{\mathrm{s}}, 0$ tempo medio Europa Centrale.

2. - L'attività sismica degli ultimi mesi rientra nella microsismicità delle zone montuose, dove la dinamica degli strati coinvolti nell'orogenesi non è ancora cessata. $\dot{L}$ a $i$ margini, ciò̀, della vera e propria storia sismica del Bellunese, e zone limitrofe, quale ci è stata tramandata dalle cronache dei grandi terremoti del passato. In dette cronache, Belluno è ricordata per $i$ disastrosi scotimenti sismici del 365, 1117, 1348, 1403 e 1511. Il fatto che le antiche cronache si limitino a citare Belluno, non va inteso nel senso che l'epicentro dei terremoti menzionati interessasse esclusivamente la citta; la citazione deve intendersi riferita al piu grosso centro abitato, in cui quei terremoti hanno prococato danni, indipendentemente dall'esatta posizione dei rispettivi epicentri. Per la prima volta, nel secolo scorso, in occasione del disastroso terremoto del 18\%3, oltre a Belluno, si citano località viciniori, quali Vittorio Veneto e l'Alpago, in cui sembra che tale terremoto abbia avuto il suo sfogo epicentrale. A questo riguardo, va osservato che è questa - di fatto - la zona dove, anche di recente, si sono verificati i piu forti terremoti che hanno, comunque, interessato il Bellunese. Il piu recente di questi il disastroso terremoto del Cansiglio del 18 Ottobre 1936 - è stato oggetto di un particolareggiato lavoro $\left(^{5}\right)$.

Come è stato detto nella precedente relazione, l'attività sismica in atto nelle località confinanti con il Vajont, assume - almeno per ora - un aspetto del tutto secondario (la manifestazione piu cospicua, sempre in senso relativo, essendo stata quella del Dicembre 1933, pure studiata da ('aloi). L'avvento di una sistematica, moderna serie di osservazioni sismografiche, ha permesso di localizzare via via questi centri di attività secondaria: Monte Pramaggiore, Col Visentin, Col (Crep) Nudo, ed altri. L̀ appunto dalle pendici del Col Nudo che è scaturita la scossa che ha allarmato, lo scorso 2 settembre, le popolazioni ai Erto, Cimolais, Soverzene, Longarone, Castellavazzo, ecc." Fig. 22. 
STAZIONE SISMICA SITA IN SPALLA SINISTRA (CORONAMENTO DIGA) presso la diga del Vajont

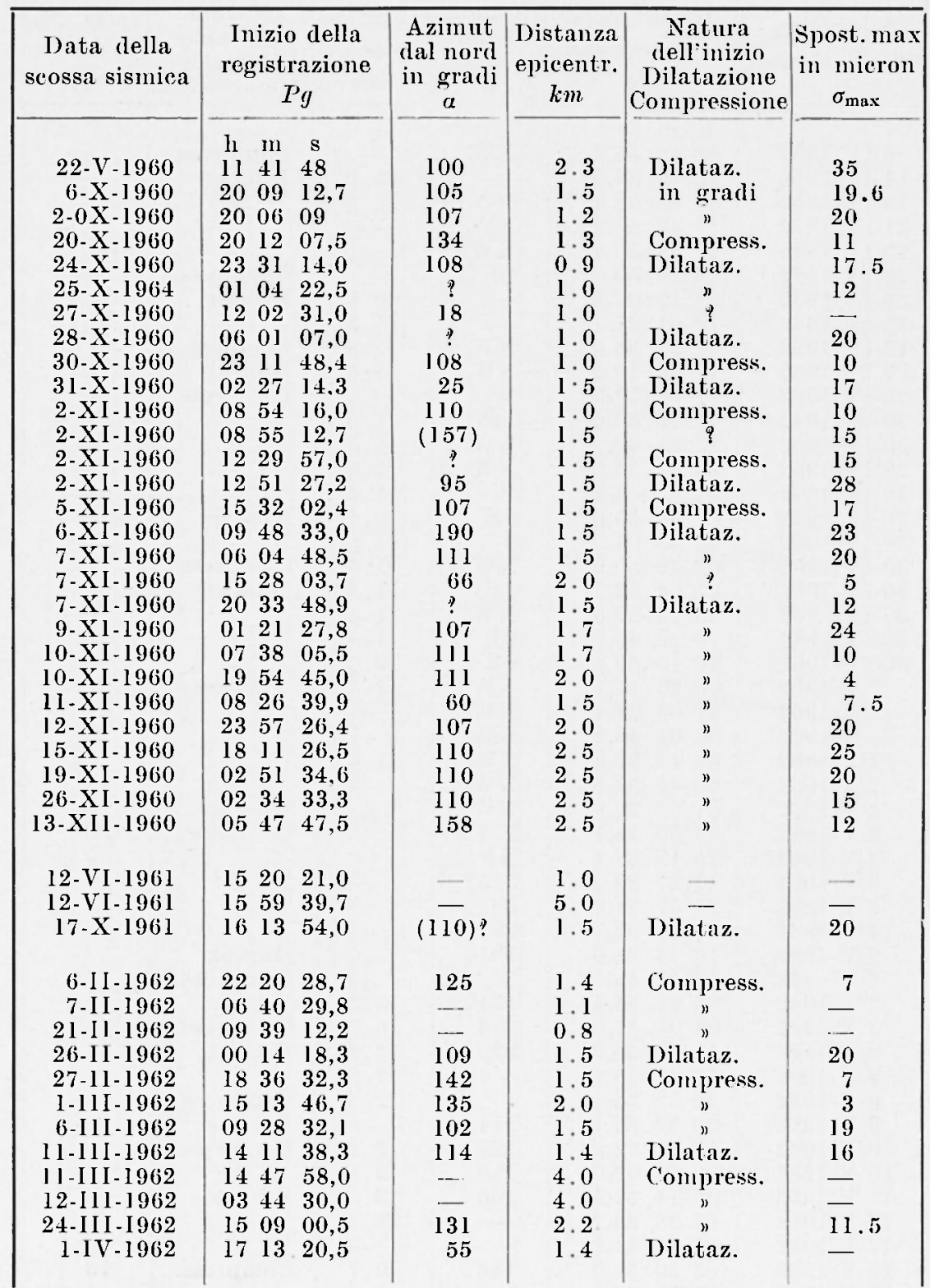

Registrazioni ottenute dal 22-V-1960 al 23-IX-1963. I'asterisco in alto a destra dellora inizio della registrazione, indica i tempi non corretti. 
(seguito)

\begin{tabular}{|c|c|c|c|c|c|}
\hline $\begin{array}{c}\text { Data della } \\
\text { scossa sismica }\end{array}$ & $\begin{array}{c}\text { Inizio della } \\
\text { registrazione } \\
P g\end{array}$ & $\begin{array}{c}\text { Azimut } \\
\text { dal nord } \\
\text { in gradi } \\
a\end{array}$ & $\begin{array}{c}\text { Distanza } \\
\text { epicentr. } \\
k m \\
\end{array}$ & $\begin{array}{c}\text { Natura } \\
\text { dell'inizio } \\
\text { Dilatazione } \\
\text { Compressione }\end{array}$ & $\begin{array}{c}\text { Spost. max } \\
\text { in micron } \\
\sigma_{\max } \\
\end{array}$ \\
\hline $6-$ IV-I 962 & $\begin{array}{ccc}\text { ll } & \text { nt } & \text { s } \\
22 & 10 & 55,3\end{array}$ & NW & - & Compress. & $\ldots$ \\
\hline $14-[V-1962$ & $\begin{array}{l}154935,0 \\
\end{array}$ & - & 25.0 & Dilataz. & - \\
\hline 19.IV-1962 & $\begin{array}{lll}05 & 09 & 02,4\end{array}$ & 305 & 1.5 & $"$ & - \\
\hline $21-1 V-1962$ & $1743 \quad 53,2$ & NW & 一 & $"$ & 3.4 \\
\hline 23 - IV-1962 & $2157 \quad 55,8$ & $\mathrm{NW}$ & - & $"$ & 140 \\
\hline 23-IV-1962 & $2324 \quad 50,5$ & 54 & 一 & Compress. & - \\
\hline $26-\mathrm{IV}-1962$ & $\begin{array}{lll}08 & 45 & 07,7\end{array}$ & - & 40.0 & Dilataz. & - \\
\hline 27 IV-1962 & $2001 \quad 27,7$ & - & 15.5 & - & - \\
\hline 29 - IV- 1962 & 125208,0 & NW & 1.5 & Compress. & - \\
\hline 29 - IV-1962 & $\begin{array}{lll}17 & 58 & 10,5\end{array}$ & $\mathrm{NW}$ & 1.5 & Dilataz. & - \\
\hline $29-$ IV- 1962 & $1758 \quad 23,3$ & W & - & Compress. & 160 \\
\hline 29 -IV-1962 & $1851 \quad 37,6$ & 145 & 1.5 & 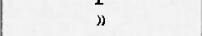 & 3.8 \\
\hline 29 - IV-1962 & $2231 \quad 40,2$ & NW & 1.5 & $"$ & - \\
\hline 29 -IV-1962 & $23 \quad 40 \quad 35,5$ & NW & 1.5 & " & - \\
\hline 30 -IV-1962 & $00 \quad 12 \quad 43,4$ & W & 1.5 & $"$ & - \\
\hline 30-IV- 1962 & $0138 \quad 35,6$ & $\mathrm{SE}$ & 1.5 & Dilataz. & - \\
\hline 30-IV-1962 & $\begin{array}{lll}02 & 38 & 01,9\end{array}$ & $\mathrm{~S}$ & 1.5 & $n$ & - \\
\hline $30-1 V-1962$ & $\begin{array}{lll}02 & 49 & 50,5\end{array}$ & NW & 1.5 & Compress. & - \\
\hline $30-1 V-1962$ & $0304 \quad 33,2$ & $\mathrm{SE}$ & 1.5 & Dilataz. & - \\
\hline $30-I V-1962$ & 131325,5 & $\mathrm{NW}$ & 1.4 & $"$ & - \\
\hline 30-IV-1962 & 162945,6 & $\mathrm{SE}$ & 1.4 & » & - \\
\hline $30-1 V-1962$ & $2045 \quad 53,0$ & $\mathrm{SE}$ & 9.5 & » & - \\
\hline I-V-1962 & $\begin{array}{lll}00 & 20 & 11,7\end{array}$ & NW & 1.4 & Compress. & - \\
\hline l-V-1962 & $\begin{array}{lll}02 & 03 & 21,4\end{array}$ & NW & - & $n$ & - \\
\hline $1-V-1962$ & $04 \quad 04 \quad 43,3$ & NW & - & $"$ & $\ldots$ \\
\hline I.V.1962 & $\begin{array}{lll}09 & 19 & 29,3\end{array}$ & NW & 1.4 & $"$ & \\
\hline $2-V-1962$ & $0041 \quad 52,5$ & $\mathrm{NW}$ & - & $"$ & 13.6 \\
\hline $2 \cdot V-1962$ & $\begin{array}{lll}01 & 55 & 20,9\end{array}$ & NW & - & Dilataz. & \\
\hline $2-V-1962$ & $18 \quad 50 \quad 48,1$ & 234 & -- & Compress. & 180 \\
\hline $2 \cdot V-1962$ & $\begin{array}{lll}19 & 15 & 38,4\end{array}$ & $\mathrm{NE}$ & - & $"$ & 4 \\
\hline $3 \cdot V-1962$ & 112723,2 & 315 & - & $"$ & - \\
\hline $3-V-1962$ & 205154,9 & $28 \mathrm{I}$ & - & $"$ & - \\
\hline $4-V-1962$ & $0611 \quad 03,7$ & 297 & - & 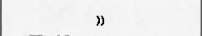 & - \\
\hline $6 \cdot V-1962$ & $1013 \quad 38,0$ & 101 & - & Dilataz. & - \\
\hline $6-V-1962$ & 164345,5 & W & - & Compress. & - \\
\hline $7-V-1962$ & $0501 \quad 25,1$ & 131 & 2.2 & Dilataz. & - \\
\hline $8-V-1962$ & $\begin{array}{llll}08 & 04 & 47.9\end{array}$ & 275 & $0 . \overline{7} 5$ & Compress. & - \\
\hline $8-V-1962$ & II $39 \quad 56,9$ & 225 & 2.8 & Dilataz. & - \\
\hline $8-V-1962$ & $1540 \quad 45,4$ & - & 2.8 & $"$ & - \\
\hline $8-V-1962$ & $2232 \quad 24,8$ & 一 & 2.8 & Compress. & - \\
\hline $9 \cdot V-1962$ & $\begin{array}{lll}00 & 52 & 27,1\end{array}$ & 214 & - & Dilataz. & - \\
\hline $10 \cdot V-1962$ & $1701 \quad 22,9$ & 228 & 2.8 & Compress. & - \\
\hline $10-V-1962$ & $\begin{array}{lll}22 & 07 & 02,9\end{array}$ & 213 & 2.8 & $"$ & - \\
\hline 11-V-1962 & 0011131,5 & 105 & 3.5 & Dilataz. & 5.3 \\
\hline $12-V-1962$ & $\begin{array}{lll}05 & 28 & 23,7\end{array}$ & - & 28.7 & - & - \\
\hline $12-V-1962$ & $0731 \quad 41,6$ & 一 & - & - & - \\
\hline $13-V-1962$ & $0330 \quad 35,0$ & 126 & 0.7 & Compress. & 15 \\
\hline I3-V-1962 & 033103,0 & 120 & 0.7 & $"$ & 4.9 \\
\hline $13-V-1962$ & $04 \quad 16 \quad 41,4$ & NW & 2.8 & Dilataz. & - \\
\hline $13-V-1962$ & $\begin{array}{lll}07 & 58 & 04,4\end{array}$ & $\mathrm{NW}$ & 2.8 & $"$ & - \\
\hline
\end{tabular}


(seguito)

\begin{tabular}{|c|c|c|c|c|c|}
\hline $\begin{array}{c}\text { Data della } \\
\text { scossa sismica }\end{array}$ & $\begin{array}{c}\text { Inizio della } \\
\text { registrazione } \\
\ulcorner g\end{array}$ & $\begin{array}{c}\text { Azimut } \\
\text { dal nord } \\
\text { in gradi } \\
a \\
\end{array}$ & $\begin{array}{c}\text { Distanza } \\
\text { epicentr. } \\
k m \\
\end{array}$ & $\begin{array}{c}\text { Natura } \\
\text { dell'inizio } \\
\text { Dilatazione } \\
\text { Compressione }\end{array}$ & $\begin{array}{c}\text { Spost.max } \\
\text { in micron } \\
\sigma_{\max }\end{array}$ \\
\hline & $\mathrm{h}$ in $\mathrm{s}$ & & & & \\
\hline $\begin{array}{l}14 \cdot V-1962 \\
14-V \cdot 1962\end{array}$ & $\begin{array}{lll}13 & 45 & 40,0 \\
1956 & 36,8\end{array}$ & $\underline{328}$ & $\begin{array}{l}1.4 \\
2.8\end{array}$ & Compress. & - \\
\hline $15 \cdot V-1962$ & $\begin{array}{lll}22 & 14 & 08,9\end{array}$ & - & $\begin{array}{l}4.0 \\
4.2\end{array}$ & - & - \\
\hline $18-V-1962$ & $01 \quad 44 \quad 58,5$ & 62 & $2 . \overline{1}$ & Compress. & - \\
\hline $18-V-1962$ & $\begin{array}{lll}07 & 28 & 01,5\end{array}$ & 65 & 2.1 & $n$ & - \\
\hline $22 \cdot V-1962$ & $\begin{array}{lll}00 & 56 & 50,2\end{array}$ & 107 & 1.4 & Dilataz. & 4.5 \\
\hline $26-V \cdot 1962$ & $1803 \quad 38,3$ & 160 & 0.7 & Compress. & 6 \\
\hline $29-V-1962$ & $\begin{array}{lll}02 & 10 & 16,7\end{array}$ & 115 & 0.7 & Dilataz. & 3 \\
\hline $30 \cdot V-1962$ & $\begin{array}{lll}04 & 30 & 26,1\end{array}$ & 44 & 2.8 & $"$ & 3 \\
\hline 12-VI-1962 & $062645,6^{*}$ & 45 & 17.0 & Compress. & 5 \\
\hline 17-VI-1962 & $173626,1 *$ & 152.5 & 0.75 & " & - \\
\hline 18-VI-1962 & $0344 \quad 52,8$ & 228 & 1.4 & " & - \\
\hline 19-VI-1962 & $\begin{array}{lll}23 & 00 & 49,0\end{array}$ & 121.5 & 1.4 & Dilataz. & 28.4 \\
\hline 3.VII-1962 & $14 \quad 14 \quad 09,2$ & - & 1.4 & Compress. & 5 \\
\hline $5-V I I-1962$ & $\begin{array}{llll}10 & 37 & 55,1\end{array}$ & - & 6.0 & Dilataz. & -- \\
\hline 5-VII- 1962 & $\begin{array}{lll}15 & 28 & 12,7\end{array}$ & ..- & 2.8 & $"$ & - \\
\hline 9.VII-1962 & $1815 \quad 41,3$ & 135 & 0.8 & Compress. & 4 \\
\hline 11-VII-1962 & $\begin{array}{lll}11 & 53 & 15,8\end{array}$ & 135 & 0.7 & 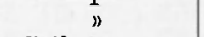 & 4 \\
\hline 12.VII-1962 & $1703 \quad 46,0$ & 35 & 1.4 & Dilataz. & - \\
\hline 12-VII-1962 & $\begin{array}{lll}18 & 43 & 19,2\end{array}$ & 326 & 1.4 & " & 3 \\
\hline 26 -VIL-1962 & 193123,5 & 122 & 1.4 & ” & 29.2 \\
\hline 1-VIII-1962 & $00+3 \quad 49,6$ & 40 & 1.4 & נ) & - \\
\hline 10.IX-1962 & $1332 \quad 12,0$ & - & 0.75 & Compress. & - \\
\hline $15-\mathrm{IX}-1962$ & $\begin{array}{lll}18 & 26 & 30,5\end{array}$ & 115 & 1.4 & 1 & 10.5 \\
\hline $15 \cdot I X-1962$ & $\begin{array}{lll}18 & 27 & 39,8\end{array}$ & - & 2.1 & ” & \\
\hline $16-\mathrm{IX}-1962$ & $\begin{array}{lll}17 & 13 & 13,8\end{array}$ & 65 & 1.4 & $»$ & 4 \\
\hline 16-IX-1962 & $1835 \quad 25,9$ & 57 & 1.4 & $»$ & 3 \\
\hline $21-\mathrm{IX}-1962$ & $1050 \quad 09,0^{*}$ & - & 17.0 & Dilataz. & - \\
\hline $21 \cdot I X-1962$ & 112929,8 & - & 16.0 & Compress. & - \\
\hline 25-IX-1962 & $08 \quad 14 \quad 57,4$ & 316 & 1.4 & Dilataz. & 4 \\
\hline 25-IX-1962 & $1851 \quad 34,3$ & 64 & 1.4 & Compress. & 2.5 \\
\hline 28-IX-1962 & $18 \quad 48 \quad 43,4$ & 159 & 0.7 & $"$ & 22 \\
\hline $28 \cdot X \cdot 1962$ & $0218 \quad 19,4^{*}$ & - & 16.5 & Dilataz. & 3.5 \\
\hline 31-X-1962 & $170424,9^{*}$ & 168 & 1.4 & Compress. & 6 \\
\hline 7-XI-1962 & $2142 \quad 28,9^{*}$ & 304 & 0.7 & Dilataz. & - \\
\hline 8-XI-1962 & $020812,3^{*}$ & - & 0.7 & Compress. & - \\
\hline $8-\bar{X} \mathrm{I}-1962$ & $0208 \quad 49,2 *$ & - & 0.7 & $"$ & - \\
\hline 8.XI-1962 & $131713,9^{*}$ & - & - & $"$ & - \\
\hline 8-XI- 1962 & $133237,0^{*}$ & - & - & $"$ & - \\
\hline 9-XI-1962 & $0657 \quad 05,4^{*}$ & 157 & 1.4 & $"$ & - \\
\hline 17-XI-1962 & $174925,3^{*}$ & - - & 17.0 & - & - \\
\hline 18-XI-1962 & $164635,7^{*}$ & - & 17.0 & Compress. & - \\
\hline 19-XI-1962 & $\begin{array}{lll}01 & 30 & 40,2\end{array}$ & - & 1.4 & Dilataz. & - \\
\hline 19-XI-1962 & $0231 \quad 41,6$ & - & 1.4 & " & - \\
\hline 8-XII-1962 & $0142 \quad 08,6$ & - & 1.4 & $"$ & - \\
\hline 8.XII-1962 & $1932 \quad 54,0$ & - & 1.4 & $"$ & - \\
\hline 14-XII-1962 & $2257 \quad 39,1$ & 137 & 0.75 & Compress. & 11.5 \\
\hline 17.XII-1962 & $1524 \quad 46,0$ & $\begin{array}{c}\text { Sparite im- } \\
\text { magini dal } \\
\text { sismo-- } \\
\text { gramma }\end{array}$ & & & 05 \\
\hline
\end{tabular}


(seguito)

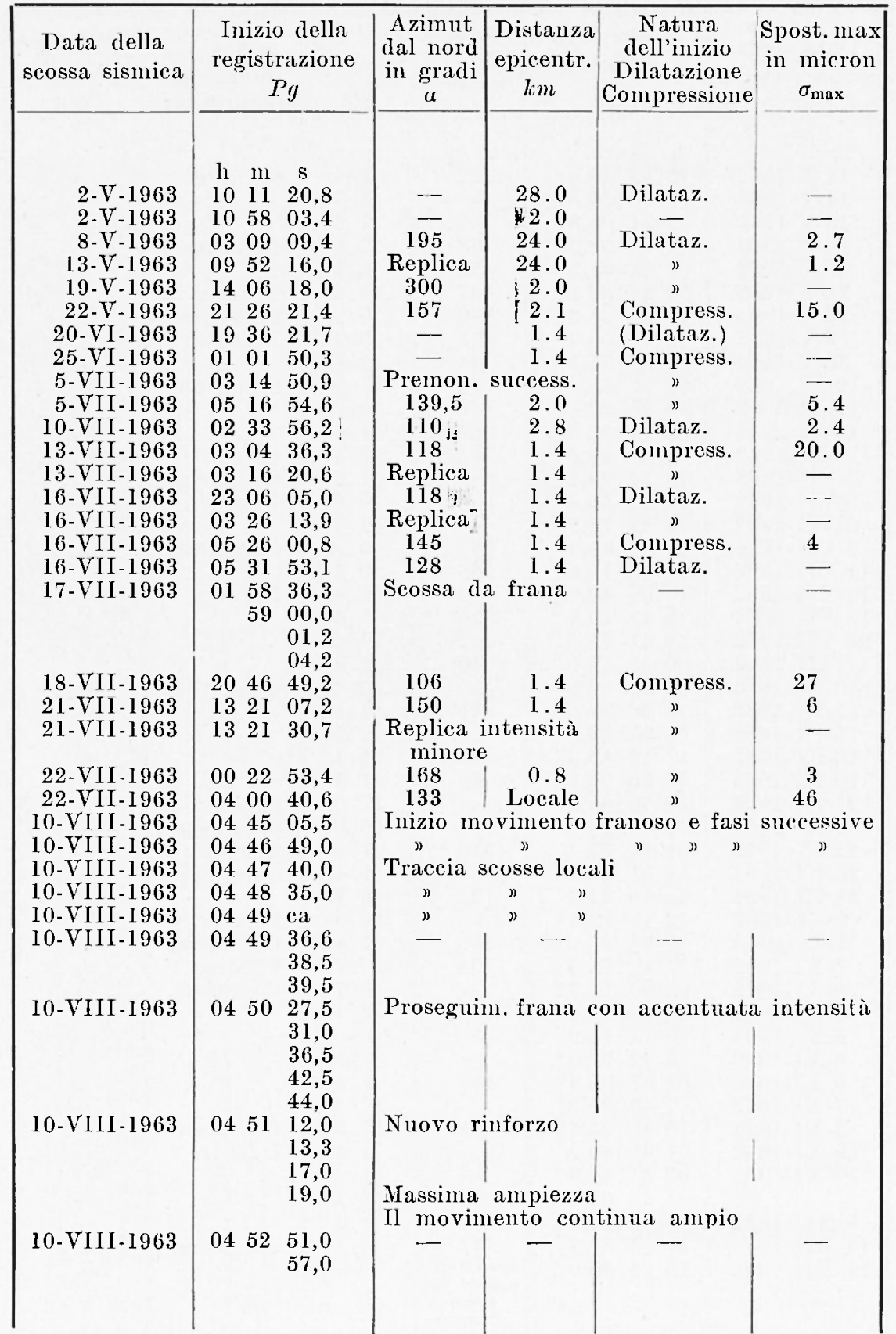


(seguito)

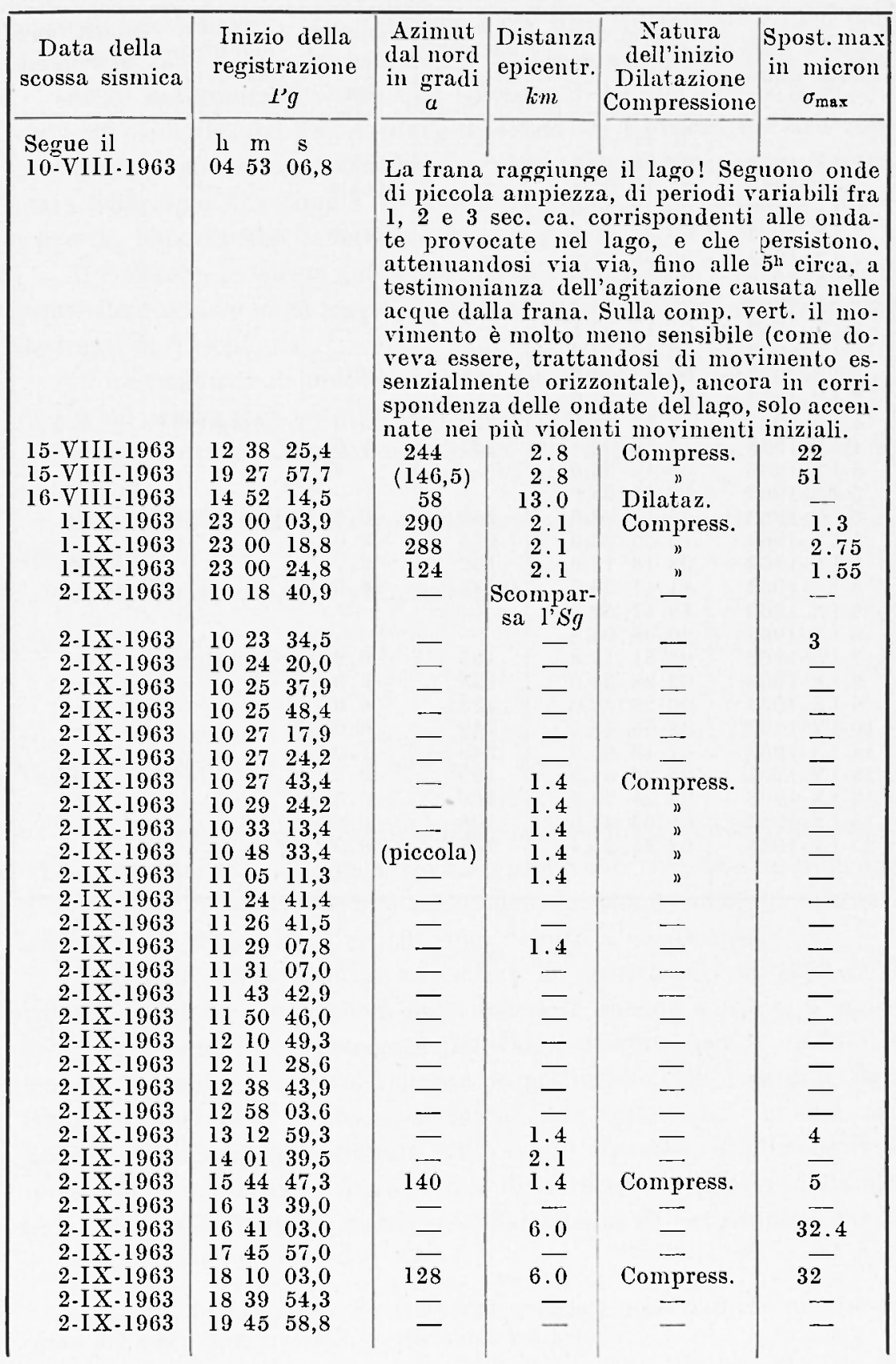


(seguito)

\begin{tabular}{|c|c|c|c|c|c|}
\hline $\begin{array}{l}\text { Data della } \\
\text { scossa sismica }\end{array}$ & $\begin{array}{c}\text { Inizio della } \\
\text { registrazione } \\
P g\end{array}$ & $\begin{array}{c}\text { Azimut } \\
\text { dal nord } \\
\text { in gradi } \\
a \\
a\end{array}$ & $\begin{array}{c}\text { Distanza } \\
\text { epicentr. } \\
k m\end{array}$ & $\begin{array}{c}\text { Natura } \\
\text { dell'inizio } \\
\text { Dilatazione } \\
\text { Compressione }\end{array}$ & $\begin{array}{c}\text { Spost.max } \\
\text { in micron } \\
\sigma_{\max }\end{array}$ \\
\hline $9 \mathrm{IX} 1063$ & li $\mathrm{m}$ s & 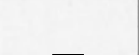 & Locole & & $\bar{c}$ \\
\hline $\begin{array}{l}2 \text {-IX-1963 } \\
\text { 2-IX-1963 }\end{array}$ & $\begin{array}{lll}23 & 03 & 10,2 \\
23 & 49 & 52,4\end{array}$ & 二 & $\begin{array}{l}\text { Locale } \\
\text { Locale }\end{array}$ & 二 & 二 \\
\hline 3-IX-1963 & $0003 \quad 36,0$ & - & & - & - \\
\hline $3-I X-1963$ & $0051 \quad 09,5$ & 一 & Locale & - & - \\
\hline $3.1 X-1963$ & $0055 \quad 53,6$ & - & - & - & - \\
\hline $3-I X-1963$ & $0056 \quad 55,6$ & - & - & - & - \\
\hline $3-\mathrm{IX}-1963$ & $\begin{array}{lll}00 & 57 & 24,9\end{array}$ & - & - & - & - \\
\hline $3-1 X-1963$ & $01 \quad 1753,1$ & - & - & - & - \\
\hline $3-1 \times-1963$ & $0544 \quad 44,2$ & - & - & - & - \\
\hline $3-\mathrm{IX}-1963$ & $\begin{array}{lll}06 & 15 & 52,2\end{array}$ & - & 2.1 & - & - \\
\hline $3-1 \times-1963$ & $0703 \quad 37,0$ & - & - & - & 一 \\
\hline $4-1 X-1963$ & $0334 \quad 08,8$ & 320 & 3.0 & Compress. & 5.5 \\
\hline $4-\mathrm{IX}-1963$ & $1057 \quad 06,1$ & 127 & 3.0 & 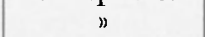 & 8 \\
\hline $5-1 \times-1963$ & $00+9 \quad 35,0$ & - & - & - & - \\
\hline 5.IX-1963 & $12+1 \quad 38,0$ & - & - & - & - \\
\hline 5-IX-1963 & 151921,6 & 150 & 6.0 & Compress. & - \\
\hline $5.1 \mathrm{X}-1963$ & 155525,0 & 215 & 4.0 & 1 & 2 \\
\hline 6-IX-1963 & $\begin{array}{lll}08 & 15 & 16,6\end{array}$ & 192 & 2.7 & " & 3 \\
\hline $6-I X-1963$ & $11+1129,9$ & 135 & 6.0 & " & - \\
\hline $6-I X-1963$ & $\begin{array}{lll}18 & 17 & 52,7\end{array}$ & - & - & - & - \\
\hline $6-\mathrm{IX}-1963$ & $\begin{array}{llll}20 & 08 & 04,8\end{array}$ & - & - & - & - \\
\hline $7-I X-1963$ & $0251 \quad 17,8$ & 135 & 6.0 & Compress. & - \\
\hline 8-IX-1963 & $0328 \quad 34,0$ & 138 & 6.0 & " & - \\
\hline 8-IX-1963 & $0528 \quad 42,0$ & 135 & 6.0 & $n$ & - \\
\hline $10-\mathrm{IX}-1963$ & $\begin{array}{lll}05 & 55 & 18,5\end{array}$ & 140 & 6.0 & $"$ & $\ldots$ \\
\hline 14-IX-1963 & $\begin{array}{lll}01 & 15 & 08,9\end{array}$ & 243 & 4.0 & " & 7.1 \\
\hline 18.IX-1963 & 050431,5 & 109 & 0.5 & " & 1.1 \\
\hline $18-\mathrm{IX}-1963$ & $0504 \quad 39,4$ & 106 & 0.5 & " & 2 \\
\hline $18-\mathrm{X}-1963$ & $0503+1,6$ & 105 & 0.5 & $"$ & 5.5 \\
\hline $23-1 X-1963$ & $\begin{array}{lll}08 & 37 & 25,4\end{array}$ & 167 & 6.0 & $"$ & 3 \\
\hline
\end{tabular}


Anche in questa, che doveva essere l'ultima relazione (ha preceduto di soli quattro giomi la distruzione della stazione sismica), si insiste sul contrasto tettonico in atto a monte della diga, al quale — sulla base delle registrazioni - viene attribuita la locale attività sismica in atto.

Il 18 Settembre 1963, in una riunione di tecnici presso la cabina comandi della diga del Vajont (dove ebbi modo di delineare, anche a voce, gli aspetti geodinamici del fenomeno in atto) (*), era stato deciso di sistemare nella diga una coppia di piccoli sismografi, allo scopo di osservarne la risposta alle sollecitazioni sismiche.

Il 7 Ottobre, assieme a due collaboratori, mi recai a Tolmezzo, dove provvedemmo allo smontaggio, e successivo imballagrgio, dei sismografi destinati al Vajont.

A mezzogiono di mercoledì 9 Ottobre 1963, il carico degli apparecehi sul camioncino era ultimato.

La partenza per Longarone (Albergo Marina) fu decisa per le tre del pomeriggio.

Poi, causa una lieve indisposizione, rimandai la partenza al mattino successivo.

Nella notte avvenne l'ultimo schianto.

II.

\section{Dopo L'Evento.}

1. - Prima di addentrarmi nell'esposizione dei successivi interventi od interpretazioni, e di abbozzare un tentativo di spiegazione di quanto è avvenuto, vorrei rispondere ad alcune obiezioni, affiorate qua e là negli squarei di relazioni, di cui sono venuto a conoscenza.

Una delle obiezioni mosse ai risultati da noi ottenuti nel 1959, nella valutazione della compattezza della roccia in sponda sinistra, a monte della diga, concerne l'elevatezza dei valori ottenuti per il modulo di Young. Va subito ricordato, a questo proposito, che sulla base della definizione di modulo di Young, i soli metodi che consentono una sua valutazione versmente approssimata, sono quelli dinamici, condizione indispensabile per eliminare l'intervento di altre aratteristiche della materia, che nulla hanno a vedere con l'elasticità. E noto infatti che la

(*) Tali, in ogni caso, da escludere qualsiasi possibilita di previsione, come del resto testimoniano le decisioni ivi prese. 
crosta terrestre si comporta come viscosa (in senso lato), quando è soggetta a lente variazioni (epirogrenesi, ecc.) e che solo azioni di forze istantance (associate a subitanee fratture) ne destano il campo elastico, in modo pressoché esclusivo. Pertanto, tutti i metodi basati su alterazioni del mezzo, ottenuto in tempi finiti, non possono condurre a valori attendibili del modulo elastico, che risulteranno di conseguenza più o meno accentuatamente inferiori a quelli effettivi. ì ciò che pressoché esclusivamente si ottiene da geologi e geotecnici, nelle loro sperimentazioni di natura statica.

Riesce poi curioso, che, a testimonianza dell'obiezione sopra riportata, venga citata una pubblicazione del prof. Müller (del 1957) in cui questi avrebbe escluso l'esistenza di rocee con elasticità tanto elevata. Evidentemente la pubblicazione del Sig. Hüller non era molto aggiomata. Fra l'altro, non va dimenticato che i valori ottenuti nel 1959 , nella zona su detta, sono precisamente dell'ordine di quelli cui eravamo pervenuti nel 1953 - con lo stesso metodo - nelle indagini della roccia al di sotto del ponte canale. In ciò deve invece riscontrarsi un argomento decisivo sulla robustezza del mezzo, fino a quella data, nella parte sottoposta ad indagine, che presentava appunto caratteri di continuità con quella sperimentata più a valle, in zona fondo diga.

Un'altra obiezione, si riferisce al decadimento osservato nel modulo elastico della zona predetta a monte della diga, nel volgere di cirea un anno. Tale fatto è ritenuto (sempre dalla stessa fonte) fortemente improbabile. Ma i fatti non si discutono. La discussione, caso mai, potrà riguardare le cause. Eे chiaro che il decadimento dell'edificio elastico di uno spessore di roccia, è strettamente legato all'intensità della causa perturbante, che potrà agire più o meno rapidamente, a parità di altre condizioni, a seconda della pressione esercitata (*).

(*) Già da un anno avevo scritto queste note, quando ebbi a leggere una relazione sull'evento del Vajont del geologo anericano George $A$. Kirsch ${ }^{\left({ }^{6}\right)}$, della Cornell University, pubblicata nel numero May-June 1965 di "Geotimes" (Rivista dell'" American Geological Institute "). Nella parte finale è scritto -a proposito della possibilita di rapido deterioramento della roceia - quanto segue:

"Vajont has tragically demonstrated the critical importance of geologic features within a reservoir and vicinity-- even though the site is otherwise satisfactory for a dam of outstanding design.

Time, in the sens of project life, is a liey lo safety and undoubtedly a principal factor at Vajont. 
Tengo poi a rettificare un'altra affermazione er'ronea, che viene sovente ripetuta.

I movimenti franosi - contrariamente a quanto è detto in alcume relazioni - non provocano terremoti, nel senso stretto che si suol dare a questo fenomeno in sismologia. Un terremoto è sempre conseguenza di un moto subitaneo, di uno schianto improvviso, quale può essere quello associato ad una frattura in un mezzo solido: solo in questo caso insorgono nel mezzo onde spaziali (longitudinali e trasversali) e quindi superficiali (di Love e di Rayleigh) Fig. 23 (registrazione ottenuta presso la stazione sismica di Pieve di Cadore).

Lo scorrere, anche violento, di un corpo di rigidità nulla o limitata, sopra un altro, provoca esclusivamente movimenti superficiali, ben (listinti e distinguibili da quelli provocati da terremoti propriamente detti. Non è affatto vero, pertanto, che le microscosse, registrate dalla

Since 1959 , nine major dams of the world have failed in some manner. It seems imperative, we recognize that

- Rock masses under changed environmental conditions can weaken within short intervals of time - days, weeks, months.

Tali conclusioni, come si vede, non potrebbero essere più affini alle mie.

E stato persino obiettato che, se i risultati dei rilievi compiuti nel 1960-61 fossero stati conformi alla realtà, l'evento sarebbe dovuto verificarsi due anni prima. Obiezione che tradisce mancanza di ponderazione e presunzione nei confronti del modo di manifestarsi dei fenomeni naturali di questi complessi, grandiosi fenomeni naturali - che sfuggono ad ogni seria possibilità di previsione. A questo proposito, si dimentica che le indagini in parola - condotte nel modo più serio ed obiettivo (tanto obiettivo da evitare ogni contatto con l'Ing. Bernabini, cui spettava l'incarico delle interpretazioni delle registrazioni ottenute con geofoni, nella fase di elaborazione dei dati ottenuti e dei risultati, risultati che collimarono perfettamente con quelli forniti dall'analisi dei dati vibrografici) - furono forzatamente parziali. Senza dubbio, qualche profondo sperone resisteva ancora sul lato sinistro; tanto da rendere necessarie altre diecine di scosse sismiche, verificatesi durante il 1961, 1962 e 1963, prima di giungere alla loro completa demolizione. Ciò è provato, del resto, dalla ripresa delle scosse, nella stessa zona, verificatesi nella prima decade del Settembre 1965 (vedi "Appendice").

E stato anche supposto che le esperienze vibrografiche compiute nel 1960-61, non siano state esattamente ripetute lungo i profili investigati nel 1959. Può darsi che qualche metro di differenza, fra i punti di scoppio e di registrazione delle due serie di indagini, vi sia effettivamente stata. Fa sorridere però sentir dire (o, peggio, trovar scritto) che pochi metri di differenza fra i riferimenti di due prove sismiche, effettuate lungo lo stesso profilo (su centinaia di metri di lunghezza!) possano portare a notevoli differenze in campo interpretativo. L'affermazione è tanto erronea, da rendere superflua ogni confutazione. 

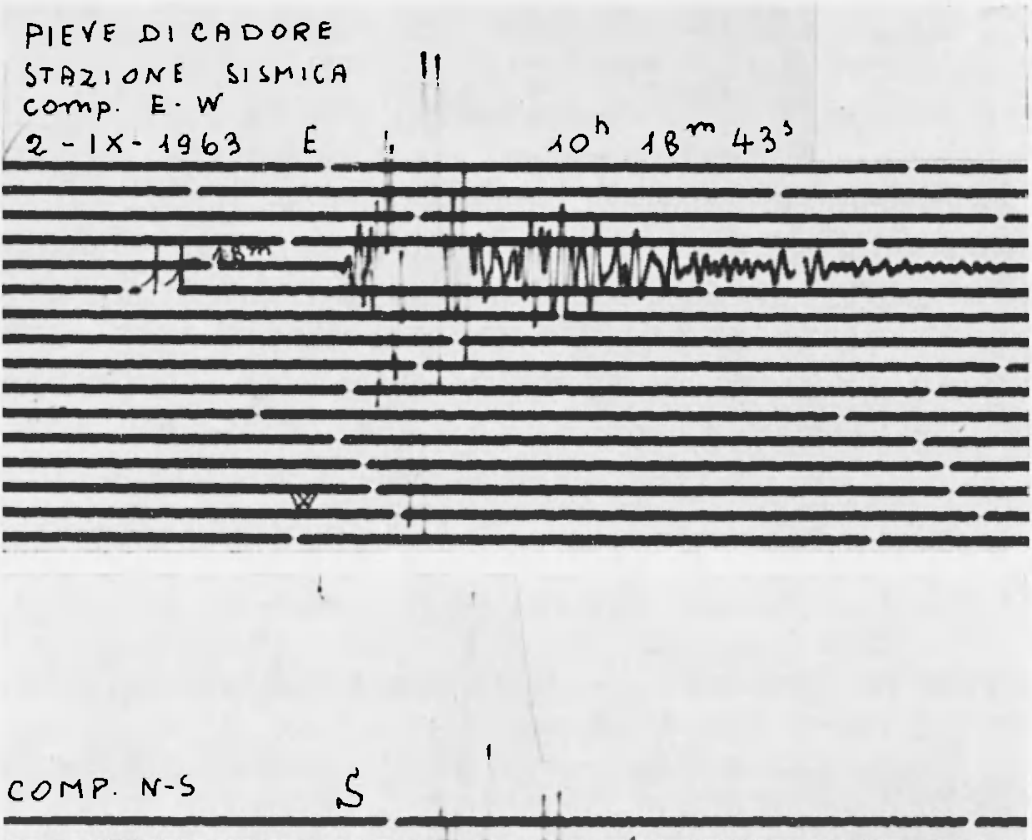

(1) $\longrightarrow$ -

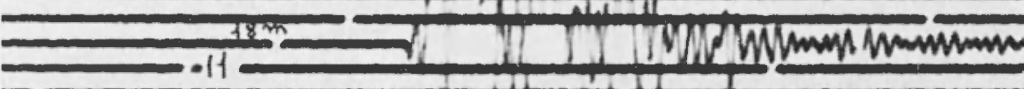
00 (20)

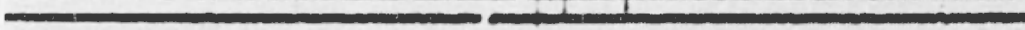

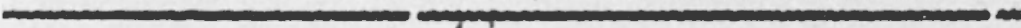
-

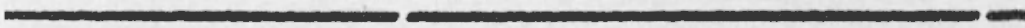

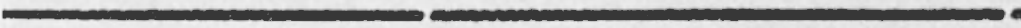

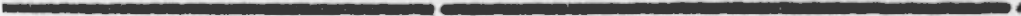

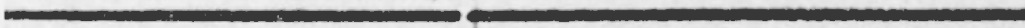
-

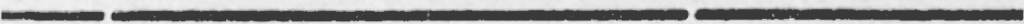
N

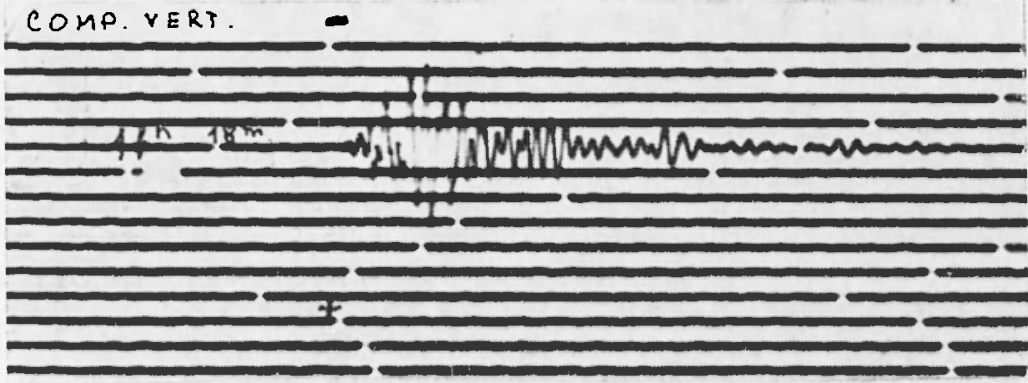

Fig. 23 - Stazione sismica di Pieve di Carlore. Scossa del 2.IX.1963 registrata sulle componenti $E W, N S$, Verticale. Azimut $155^{\circ}$; distanza epicentrale $17 \mathrm{~km}$; spostamento massimo $15 \mu$. 


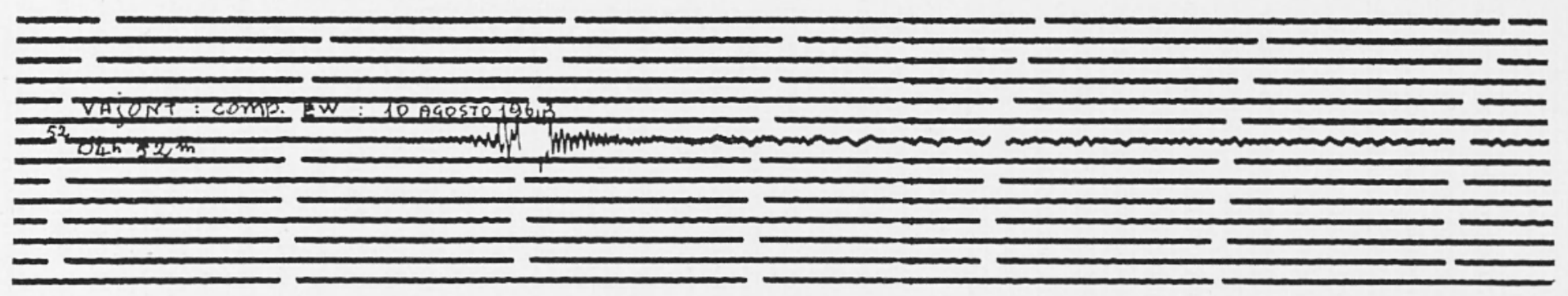

Fig. 24 - Stazione sismiea del Vajont. Ultima fase della frana parziale, in sponda sinistra del Vajont, del 10 Agrosto 1963. La serie di oscillazioni di periodo relativamente lungo da 8 secondi ca a 2 sec ca, registrate dal $53^{\mathrm{m}}$ in poi, furono determinate dalle ondate conseguenti al precipitare del materiale di frana nel lago. 
stazione sismica del Vajont, fossero determinate da frane superficiali. Le microscosse erano conseguenza esclusiva di fratture, producentisi alla base dell'ammasso di materiale, scomnesso o comunque diffusamente frantumato, che soviastava sulla sponda sinistra del Vajont, a monte della diga; in un mezzo quindi ancora compatto, elasticamente resistente.

Ciò è provato dalla casistica più varia. Le variazioni di pressione associate a cicloni transitanti sui mari, non provocano terremoti, ma oscillazioni regolari nel mezzo solidlo, che si propagano a notevole distanzal (agitazione microsismica). I marosi che battono violentemente contre le sponde roeciose a picco sul mare destano nel mezzo solido, movimenti superficiali, costituenti un altro aspetto della così detta agitazione microsismica. Un altro tipo di agitazione microsismica è quella legata al soffiare dei venti contro ostacoli sorgenti dal suolo, ecc. La fase esplosiva dei vulcani, caratterizzata da esplosione dei gas magmatici nei condotti craterici, con conseguente violento rapido scorrere del magma lungo le pareti dei condotti stessi, non provocano terremoti, ma lungo seguito di oncle di tipo superficiale: cosi, p. es., in occasione delle fasi esplosive dell'Etna, a Catania (alla distanza di $40 \mathrm{~km} \mathrm{cal)} \mathrm{vengono} \mathrm{registrate} \mathrm{lunghe}$ serie di onde superficiali di Love ( $\left.{ }^{7}\right)$.

Del resto, nel caso specifico, valgono le registrazioni, ottenute alla stazione sismica del Vajont, in caso di microscosse o di frane. Mi limito a riportare la registrazione di und microscossa locale e di una frana, verificatasi il 10 Igosto 1963 . La registrazione associata alla frana consiste di lunghe serie di viblazioni, esclusivamente costituite di movimenti di superficie: mancano, in modo evidente, le perturbazioni spaziali (longitudinali e trasversali) (Fig. 24).

Pertanto, le microscosse non provenivano affatto da frane parziali, ma da fratture nel mezzo solido.

2. - Aruti a disposizione i sismogrammi ottenuti a Pieve di Cadore, Tolmezzo e Somplago (Figg. 25, 25a, 25b) nella notte fra il 9 a il 10 Ottobre, feci una prima interpretazione - forzatamente parziale nelle sue conclusioni - , che riassunsi in una breve relazione inviata, in data 27 Ottobre 1963, all'Ufficio Misure Dighe dell'ENEL di Venezia. La riporto qui di seguito, anche se alcune affermazioni in essa contenute (con particolare riguardo alla natura delle onde superficiali) saramno suecessivamente modifieate, in seguito a più ampia ponderazione e a magrgior copia di dati d'osservazione.

"Il contrasto tettonico - di cui si è fatto cenno nelle tre precedenti relazioni - in atto in sinistra, immediatamente a monte della diga del 


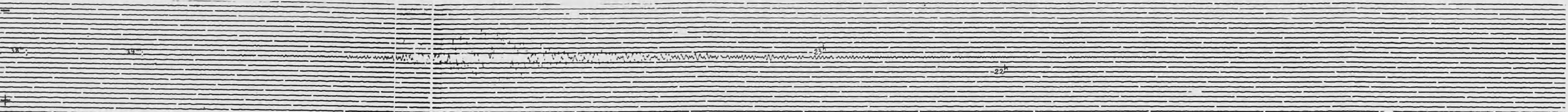

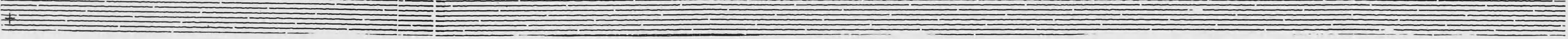
(1)

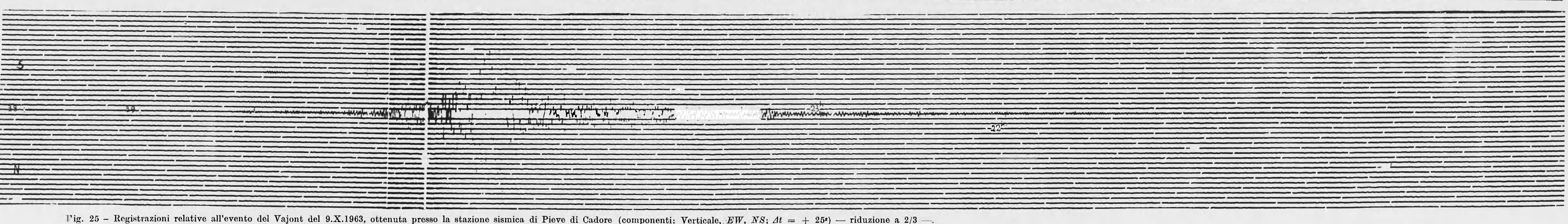

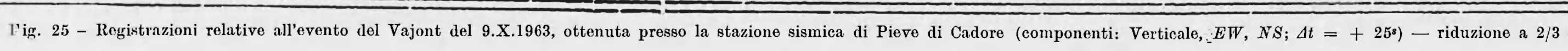

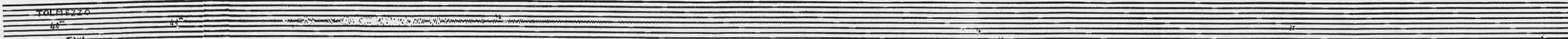

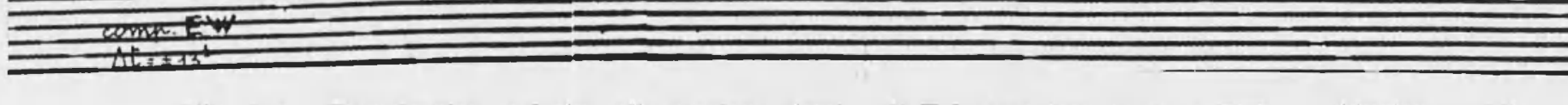



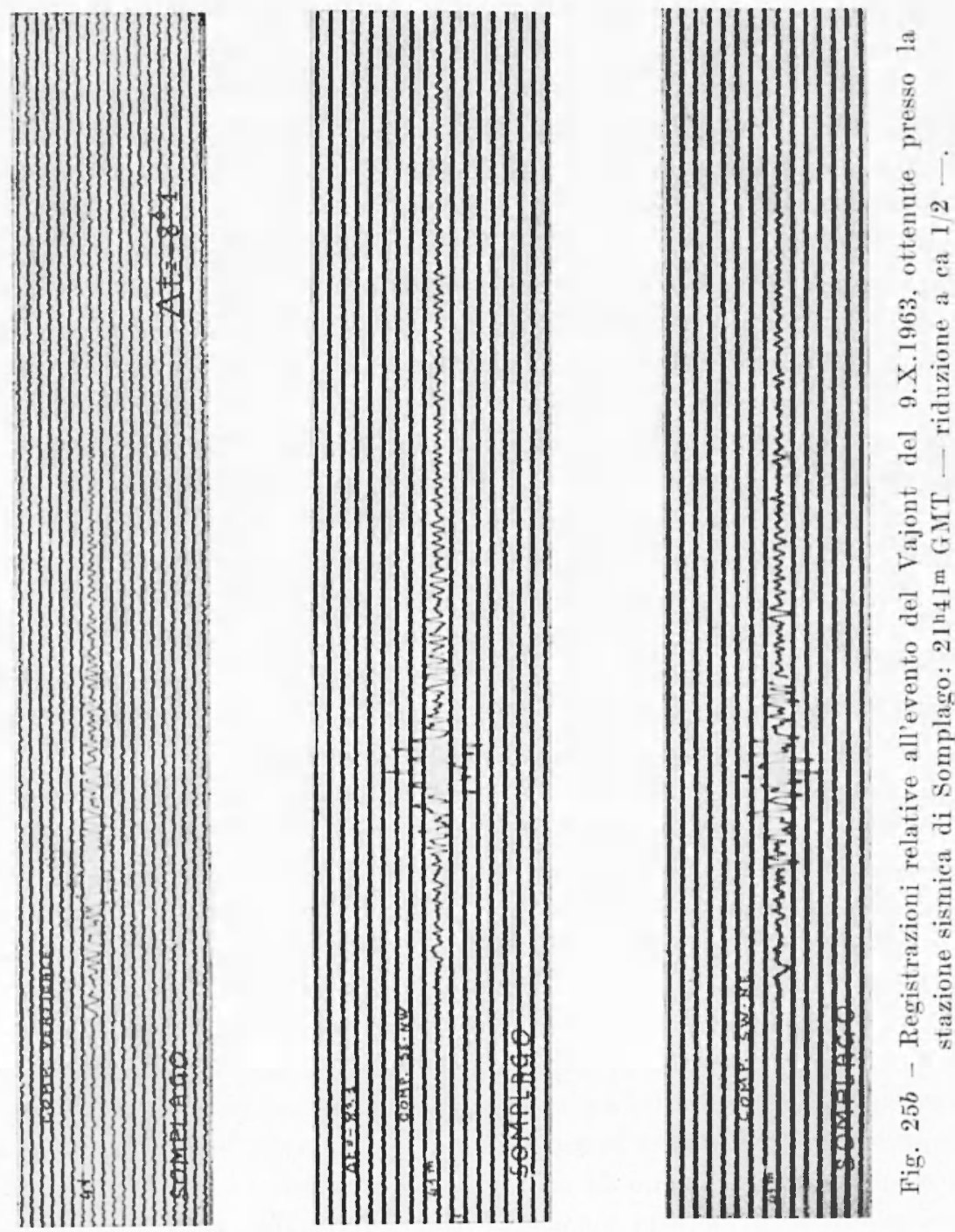
Vajont, è sfociato nell'immensa frana della sera del 9 Ottobre; l'evento, solamente adombrato come dannata ipotesi verso la fine alla relazione $I I$, si i: verifieato di proporzioni al di là di ogni più pessimistica previsione.

Esso si è realizzato sotto forma di frana: non è stato precrduto da nessuna perturbazione sismira. Di riò fanno inconfutabile testimonianza le registrazion isismografiche ottenute a Pieve ai Cadore (distanza 1\% lim ca), Tolmezzo (distanza jo lim ca) e Somplago (distanza $5.5 \mathrm{~km} \mathrm{ca}$ ). Di esse si archludono copie. Nel caso di scossa sismica, la registrazione avrebbe avuto a Pieve l'aspetto dei sismogrammi riportati nelle Figg. 2t e 2toss (tov. TIII) di "Aspetli della dinamica di rocee, ecc." (vedi qui Fig. 23), o di poco diverso (un maggiore intervallo fra le onde longitudinali e trasversali) sarebbe stato per Tolmezzo e Somplago. Nella fase dell urto della massa franata contro la momtagna in sponda destra, ha avuto origine un movimento sismico, di evidente carattere supreficiale, che si è propagato a distanza appunto sotto forma di terremoto superficiale. A Roma (alla distanza di him 480) si è avuta infalti la sola registrazione di onde superficiali ai Love (L) e ai Rayleigh (K); e lo stesso si è verificato a Nessina, alla distanza Ii $\mathrm{km} 940$.

L'energia associata al movimento sismico, conseguente alla frana, è stata valutata - sulla base delle registraziomi ottenute a Roma - dell'ordine ai $2.10^{18}$ erg (pari ciò̀ a circa la quattrocentesima parte dell'energia sviluppata da una bomba atomica ordinaria, tipo Hiroshima).

Dopo la frana, a luttogyi (26.X.1963), una sola scossa di terremoto $\grave{c}$

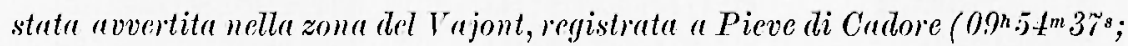
distanza $1 \% \mathrm{lim}$ ca), a Tolmezzo (distanza $53 \mathrm{lim}$ ca) e a Somplago (a 09.54 .4 ; (listrenza $55 \mathrm{~km} \mathrm{col})$.

A mis avviso, nella zona caratterizzata dalle dilatazioni (vedi precedenti relazioni) si è andato approfondendo il solco lungo oui le stratificazioni superfiriali cedevano (quesi si fosse trattato ai una micro-geosinclinale), mentre lungo le pendici del Toc si androva arcentuando il movimento positivo del sottosuolo, come è provato dal gran numero di piccole scosse in detla zona caratterizata da psclusiva compressione (spinta verso l'esterno). Alla fine il monte - forse animato da un residuo di orogenesi - si è letteralmente scrollato di dosso tutla la sovrastante massa incoerente, come conseguenza dell'ulteriore codimento del fondo di quella che abbiamo definito la microgeosinclinalen.

Pochi giomi dopo, inviai un'altma breve relazione, in eui si esponevano alcune ronsiderazioni, consexuenti all'osservazione della planimetria dellar zona, dopo l'evento. Fecola: 


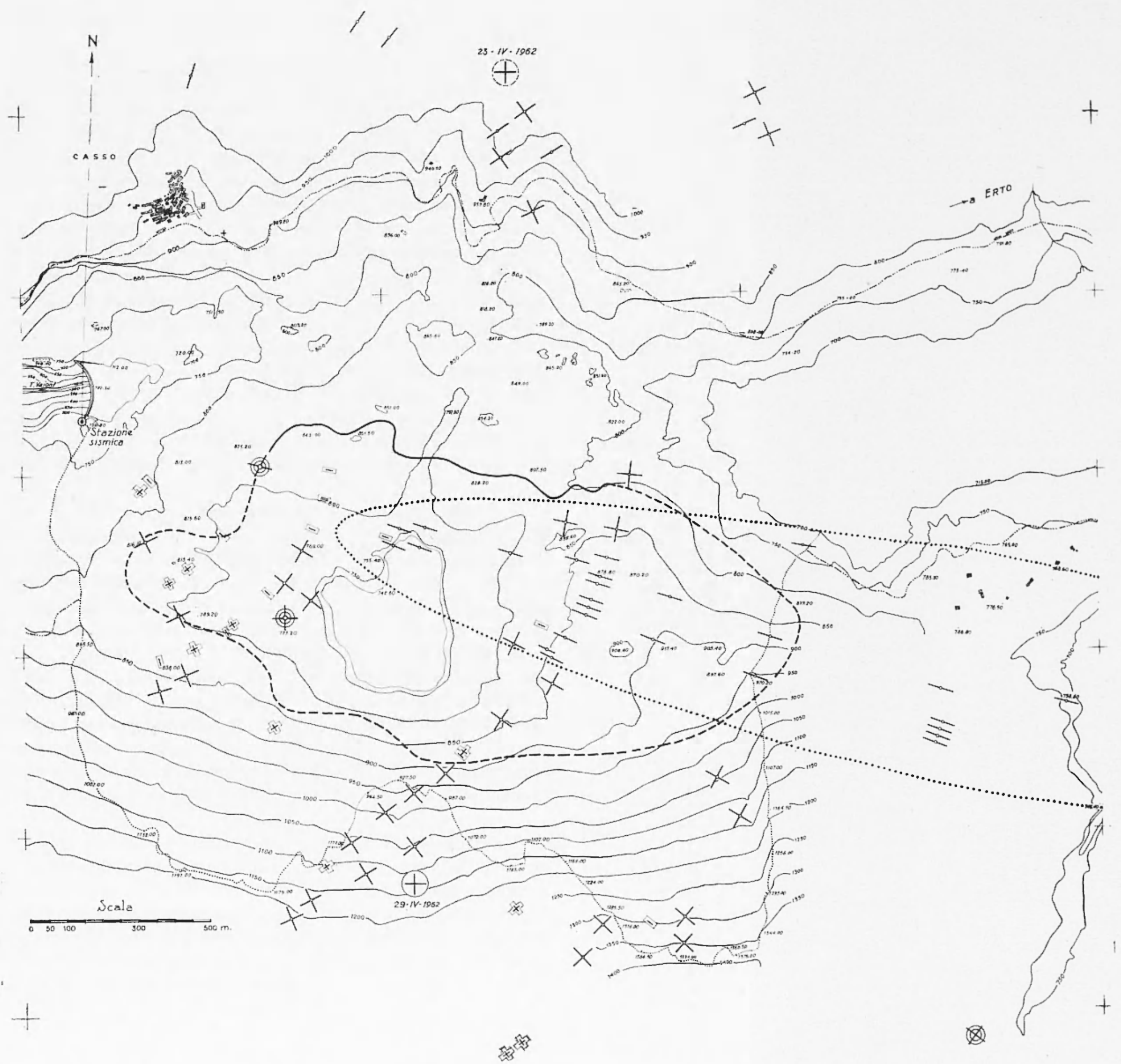

Fig. 26 - Planimetria della stretta del Vajont subito dopo l'evento. I'segni - e +i incorniciati, si riferiseono agli epicentri di alcune delle scosse verificatesi nella zona durante il periodo sismico avutosi nella prima decarle del Settembre 1965 (redi "Appendice"). 
"A complemento della mia ultima relazione sul disastro del Tajont, ritengo utili alcune considerazioni sulla nuova fisionomia assunta dalla valle, nella zona interessata dalla frana del 9 Ottobre 1963.

In data 13 Ottobre, per incarico dell'ENEL, è stato eseguito un rilievo aerofotogrammetrico dall'I.R.T.A. di Milano. Un confronto di tale rilievo con l'andxmento precedente la frana, mostra che il massimo accumulo di materiale si ̀̀ avuto nella zona caratterizzata dalla maggior frequenza delle scosse sismiche, dovute a brusco abbassamento (dilatazione). Il richiamo maggiore di materiale si è avuto quindi dove il fondo aveva ceduto, come conseguenza delle fratture verificatesi negli ultimi anni. Nella zona di contatto fra la parte in sprofondamento e quella in sollevamento (quest'ultima individuata dalla frequenza delle compressioni + ), in località prossima alla diga, l'azione di allontanamento del materiale franoso sovrastante è state massima, tanto da dar ivi origine ad un lago transitorio, la cui superficie esterna risultava il 13 Ottobre a quota $\$ 210$ (Fig. 26). Ciò fa ritenere - tenuto debito conto della posizione nettamente a monte rispetto la diga - che in questa zona deve essersi verificato il massimo cedimento, a conclusione del gioco tettonico in atto, che mostrava ivi il massimo contraston.

In un successivo esame delle probabili cause prime, contenuto in un'ulteriore relazione inviata all'Ufficio Misure Dighe, mi soffermo sulla successione degli avvenimenti, ricostruita in base alle registrazioni ottenute a Pieve di Cadore, Tolmezzo e Somplago.

Sostanzialmente, in detto esame, si espone l'ipotesi clie - come avevo già prospettato in una delle mie relazioni del 1961 - il deterioramento della roccia di base in sponda sinistra del bacino del Vajont, a monte della diga, sia da ascriversi alle microscosse che si emano verificate in questa zona durante il 1960. Per quanto concerne il disturbo tettonico, rivelato dalla stazione sismografica, mi domandavo inoltre se esso fosse da ritenersi in atto da sempre (in senso stretto) o se invece si dovesse ritenere associato ad una recente ripresa, forse legata all'azione dirompente delle cariche di esplosivo, fatte brillare nella fase iniziale dello sbancamento.

Ora, dopo l'evento, non posso escludere una certa influenza delle vibrazioni, provocate da dette esplosioni, atta a rompere un precario equilibrio nella zona, ridando vita ad un fenomeno naturale, temporaneamente assopito. Ed anche gli effetti degli invasi vanno presi in considerazione: l'azione di disturbo ad essi legata, ha probabilmente contribuito ad accentuare e ad accelerare la fase di rottura sul fondo della zona. Fatto, questo, constatato in casi amaloghi $\left({ }^{8}\right)$. 
Circa la profondità media, alla quale si verificavano le microscosse nella zona del contrasto tettonico, va osservato che una parte di esse, corrispondenti a dilatazione, ebbero il loro ipocentro più o meno nettamente al di sotto della quota della stazione sismica (q. 725); quelle corrispondenti a compressione sono invece provenienti nella generalità, da quote superiori.

Queste osservazioni concorrono a rafforzare l'ipotesi che attribuisce il contrasto tettonico verificatosi sulle pendici del monte Toc ad un residuo di orogenesi.

3. - La prima visione delle registrazioni ottenute a Pieve di Cadore, Tolmezzo e Somplago mi aveva subito convinto che non mi trovavo alla presenza di "sismogrammi", nel senso che noi diamo alla sequenza di impulsi provocati da un terremoto; e, tanto meno, di sismogrammi relativi ad un terremoto, che avrebbe dovuto originare a soli $17 \mathrm{~km}$ da Pieve di Cadore. Il fatto, più che eccezionale, si rivelava realmente nuovo.

Tentai ugualmente un'interpretazione, che esposi sia pure con qualche riserva. In essa accennavo, fra l'altro, alla possibilità di un piccolo terremoto, verso le $22^{\mathrm{h}} 41^{\mathrm{m}} 40^{\mathrm{s}}$. In realtà, specie nella prima parte, la ricostruzione dell'evento, fatta in un primo tempo sulla base delle registrazioni ottenute a Pieve di Cadore, va sostanzialmente mutata. Nel frattempo, infatti, un'accurata indagine delle registrazioni ottenute in una trentina di stazioni europee, mi ha permesso di chiarire - nei suoi fatti essenziali - il meccanismo che ha condotto alla violenta, improvvisa estromissione di circa 20 milioni di metri cubi d'acqua dalla riserva del Vajont.

L'elaborazione delle registrazioni ottenute in Europa (Figg. 27), fino a circa $2.500 \mathrm{~km}$ di distanza, confermano-in modo definitivo-che non vi fu terremoto al Vajont. In tutte le registrazioni, con sismografi a medio periodo (da 2 a $10 \mathrm{sec}$ ca), predominano due gruppi di oscillazioni trasversali - il secondo di maggiore ampiezza - separati fra loro da un intervallo di 30-32 sec. Il periodo medio di dette oscillazioni è dell'ordine di 6-7 sec. Esse risultano orlate di vibrazioni più rapide (1 sec di periodo ca), appena percettibili in corrispondenza del primo gruppo, più ampie a cavallo del secondo. In strumenti a più lungo periodo proprio (come i Galitzin e i Galitzin-Wilip), i due gruppi presentano periodi medi nettamente magriori (15s in media), mentre i sismografi a più lungo periodo proprio (come gli "Ewing-Press" di Trieste, Stoccarda, Strasburgo, L'Aquila, ecc.) registrano onde con periodi di 30 e più secondi, separate dall'intervallo di tempo su detto. Per le stazioni, più lontane (come Toledo 
e Umea) con sismografi a lungo periodo, i periodi delle onde registrate si aggirano intorno ad un minuto primo, ciò che porta a confondere l'uno nell'altro gruppo, accomunati in un solo treno d'onde.

I sismografi a breve periodo (dell'ordine del secondo, o meno) non regristrano che gruppi di vibrazioni, quali possono aversi in tracce di terremoti vicini.

Ad ogni modo, una cosa va sottolineata: terremoti ad origine vicina non provocano, già alle più brevi distanze, oscillazioni del tipo sopra accennato e di quel periodo. La causa di quelle oscillazioni va ricercata nel loro meccanismo-origine: due autentici colpi di maglio sulla crosta terrestre, a poco più di mezzo minuto l'uno dall'altro, il secondo sensibilmente più violento del primo. La crosta terrestre ha risposto alle due enormi mazzate, come può rispondere un disco indefinito stratificato, colpito a martellate nel punto centrale: con le oscillazioni proprie, cioè, delle stratificazioni componenti e della crosta in tutto il suo spessore. Ciò spiega i lunghi periodi osservati.

Torniamo alle registrazioni di Pieve di Cadore. Esse iniziano visibilmente verso le $22^{\mathrm{h}} 38^{\mathrm{m}}$; con soste, e sempre più vistose riprese, continuano fino alle $22^{\mathrm{h}} 41^{\mathrm{m}} 30^{\mathrm{s}}$ (secondo più, secondo meno). Queste preliminari vibrazioni non possono essere attribuite a franamenti parziali, esterni (con rotolamento di materiale sciolto, su materiale incoerente, di notevole spessore, elasticamente pressoché inerte): solo materiale fortemente compresso, strisciante su roccia può provocare vibrazioni trasmissibili ampiamente fino a Pieve di Cadore $o$, in misura più ridotta, a Tolmezzo $(52,5 \mathrm{~km})$ o a Somplago $(55 \mathrm{~km})$ : tali vibrazioni, pertanto, vanno attribuite a frane interne. Va osservato, a questo riguardo, che le precedenti frane parziali di superficie, mai sono riuscite ad interessare i sismografi della stazione sismica di Pieve di Cadore (la più vicina), nemmeno quella del 4 Novembre 1960. D'altronde, i testimoni dell'evento e gli avvenimenti ad esso collegati, confermano che la catastrofe - contro ogni possibilità di previsione — si è presentata in fulminea successione di tempi e di fenomeni. Ritengo che le perturbazioni, registrate con tanto anticipo a Pieve di Cadore, siano quindi da attribuire - come si è detto - a scorrimenti interni; ciò̀ a zone in movimento lungo la superficie limite rocciosa del Toc. Le fratture avvenute sul fondo, avevano probabilmente eliminato gli ultimi ostacoli al convogliamento del materiale sovrastante verso la sottostante zona, che possiamo definire caratterizzata da notevole difetto di massa (di varia interpretazione: da "interstizi " fra due monti contigui, a regione in lento sprofondamento). Solo a scorrimenti interni. di materiali compressi, costretti a moti lungo una superficie solida, può 
essere associata l'energia sufficiente alla propagazione di moti elastici. Non vedo altra via per spiegare la sequenza di micromovimenti, che si sono susseguiti a Pieve di Cadore a partire dalle $22^{\mathrm{n}} 38^{\mathrm{m}}$, ma che localmente devono essere originati alquanto prima, aumentando via via d'intensità, per raggiungere soltanto verso quell'ora l'energia sufficiente ad impressionare i sismografi funzionanti a circa $17 \mathrm{~km}$ di distanza.

Ora quindi si può ritenere che il cedimento che si era verificato sulla sinistra del Vajont, a quota inferiore a quella del fondo del bacino, aveva finito per trascinare a sé la massa sovrastante di materiale sconnesso. Ad un certo punto l'ammasso intero, facendo perno sulla base inferiore, ha ruotato verso l'altra sponda sulla quale si è al fine letteralmente rovesciato. Ciò è avvenuto verso le $22^{\mathrm{h}} 41^{\mathrm{m}} 30^{\mathrm{s}}$, come è provato dalla dromocrona delle onde trasversali, interessanti - come oscillazioni libere-lo strato esterno della crosta terrestre. A Pieve di Cadore, un'oscillazione longitudinale del mezzo arriva alle $22^{\mathrm{n}} 41^{\mathrm{m}} 33^{\mathrm{s}}$ circa, seguita, dopo tre secondi circa, da oscillazioni più ampie, forse trasversali. Sia per la brevità del periodo strumentale, sia per la breve distanza di Pieve di Cadore, ivi ancora non appaiono le onde di $5^{s} \mathrm{ca}$, che vengono invece chiaramente registrate a Somplago $(55 \mathrm{~km})$.

I'improvvisa serie di ampie, rapide vibrazioni che comincia alle $22^{\mathrm{h}} 41^{\mathrm{m}} 45^{\mathrm{s}}$ va probabilmente attribuita alla valanga d'acqua in uscita, violentemente e improvvisamente proiettata contro la parete rocciosa. verso Casso. Queste oscillazioni durano una diecina di secondi; dopo qualche secondo di movimenti attenuati, verso le $22^{\mathrm{h}} 42^{\mathrm{m}} 02^{\mathrm{s}}$, inizia la serie più ampia e duratura di rapide vibrazioni, serie che è da ritenere corrisponda alla caduta dell'enorme massa d'acqua nella forra del Vajont, da un'altezza di alcune centinaia di metri. E a questa caduta che va ascritta la seconda "mazzata ", nettamente più forte della prima e più ampiamente registrata in tutta Europa. Le ampie rapide vibrazioni, associate a questo impatto, mascherano nelle vicine stazioni con sismografi a piccolo periodo proprio (come Pieve di Cadore, Tolmezzo ed altre), la reazione delle stratificazioni della crosta in libera oscillazione: cosi a Somplago, dove l'oscillazione di $5^{\mathrm{s}}$ relativa al primo colpo subito dalla crosta appare invece pressoché immune da perturbazioni (Fig. 25b), le rapide vibrazioni essendo già state assorbite dal mezzo. Rapide vibrazioni orlano la reazione della crosta al secondo impatto $\left(6^{\mathrm{s}-10 \mathrm{~s})}\right.$ in tutta Europa, per gli apparecchi a breve, medio periodo ("*).

$\left(^{*}\right)$ Valutai in $50^{\mathrm{s}}$ ca. da $22^{\mathrm{h}} 42^{\mathrm{m}} 05^{\mathrm{s}}$ a $22^{\mathrm{h}} 42^{\mathrm{m}} 55^{\mathrm{s}}$ ca. la corsa dell'acqua nella forra del Vajont. La velocità apparente che ne consegue può sembrare eccessiva. Va però osservato che la caduta dell'acqua dev'essersi verificata 

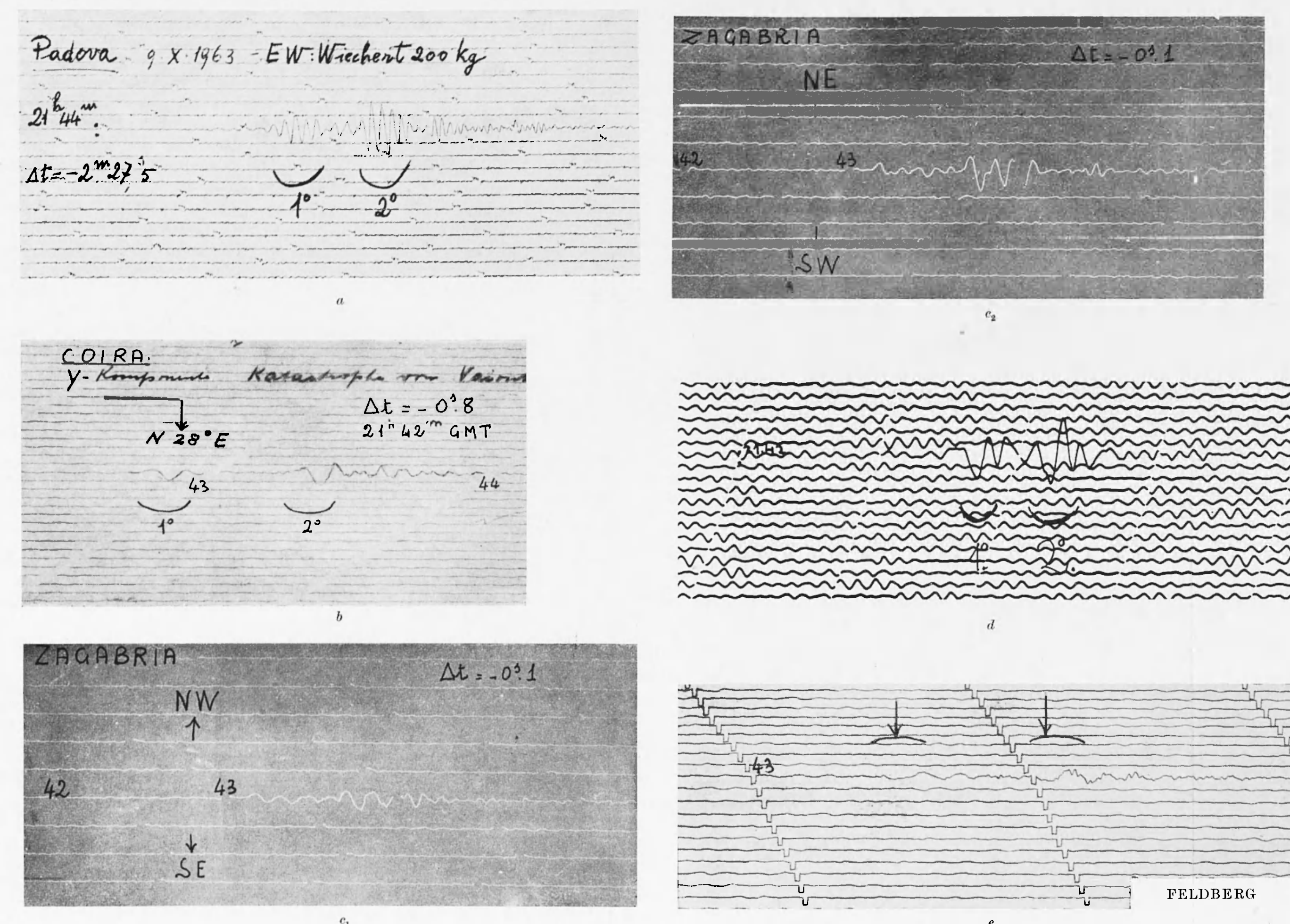
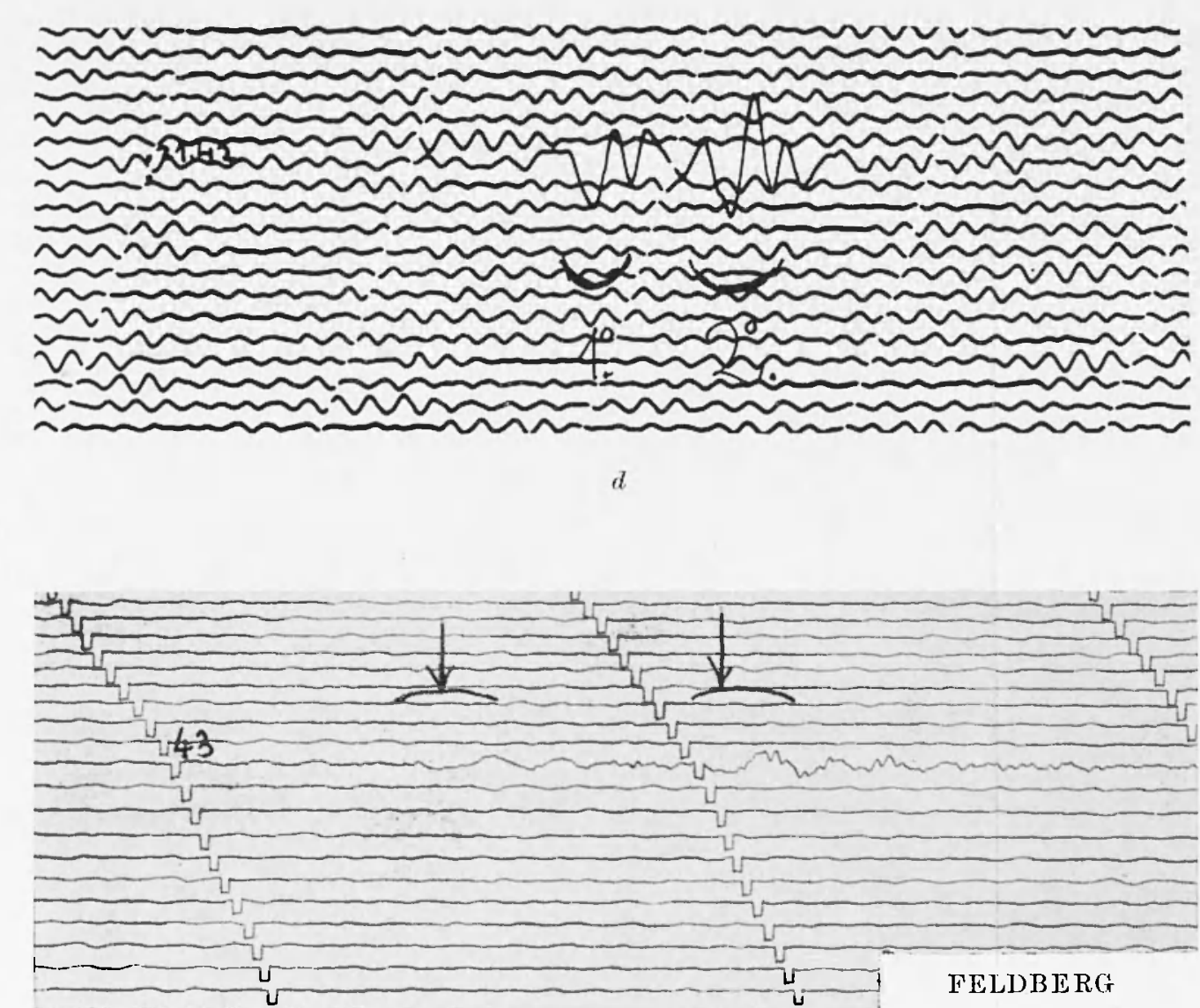
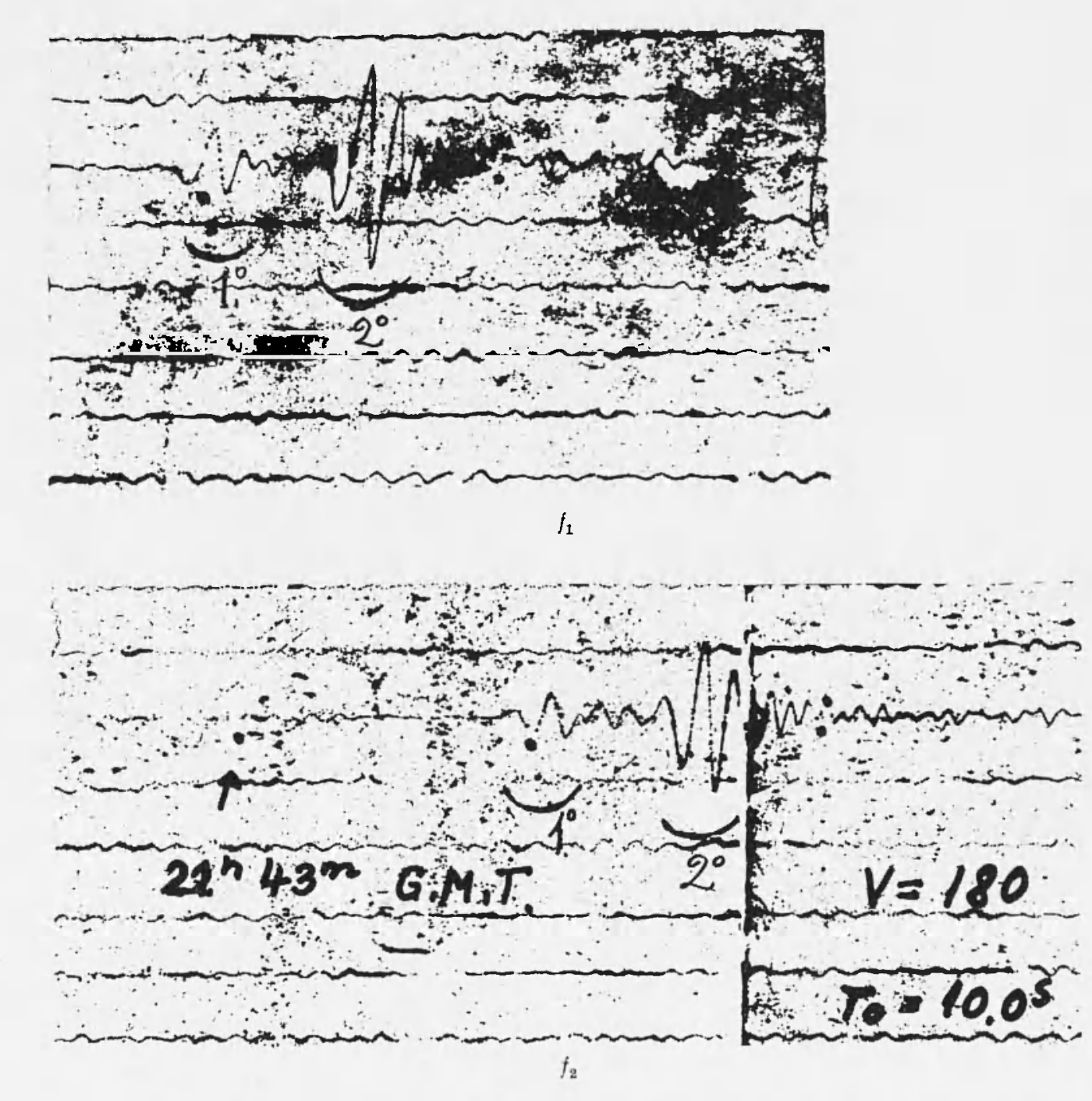

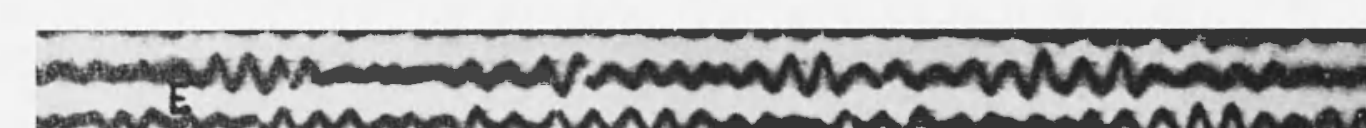
minnminn (1) minmminmminn mixnn N-

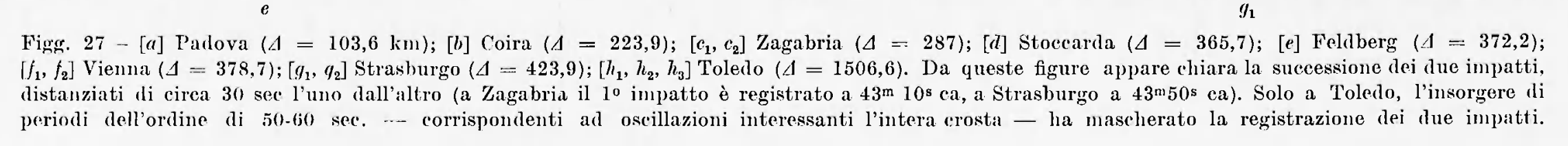

Munimin mom m.2.

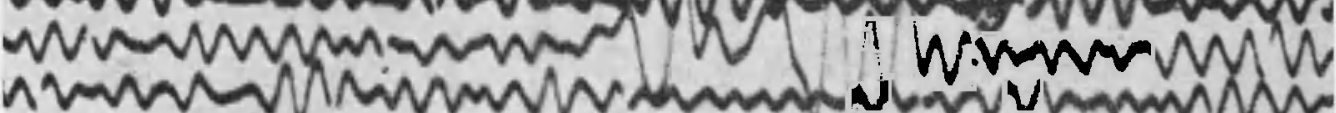

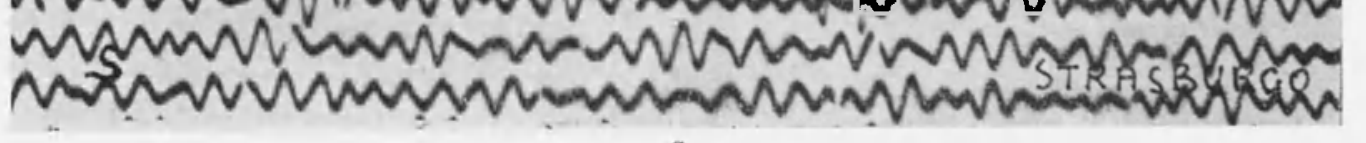

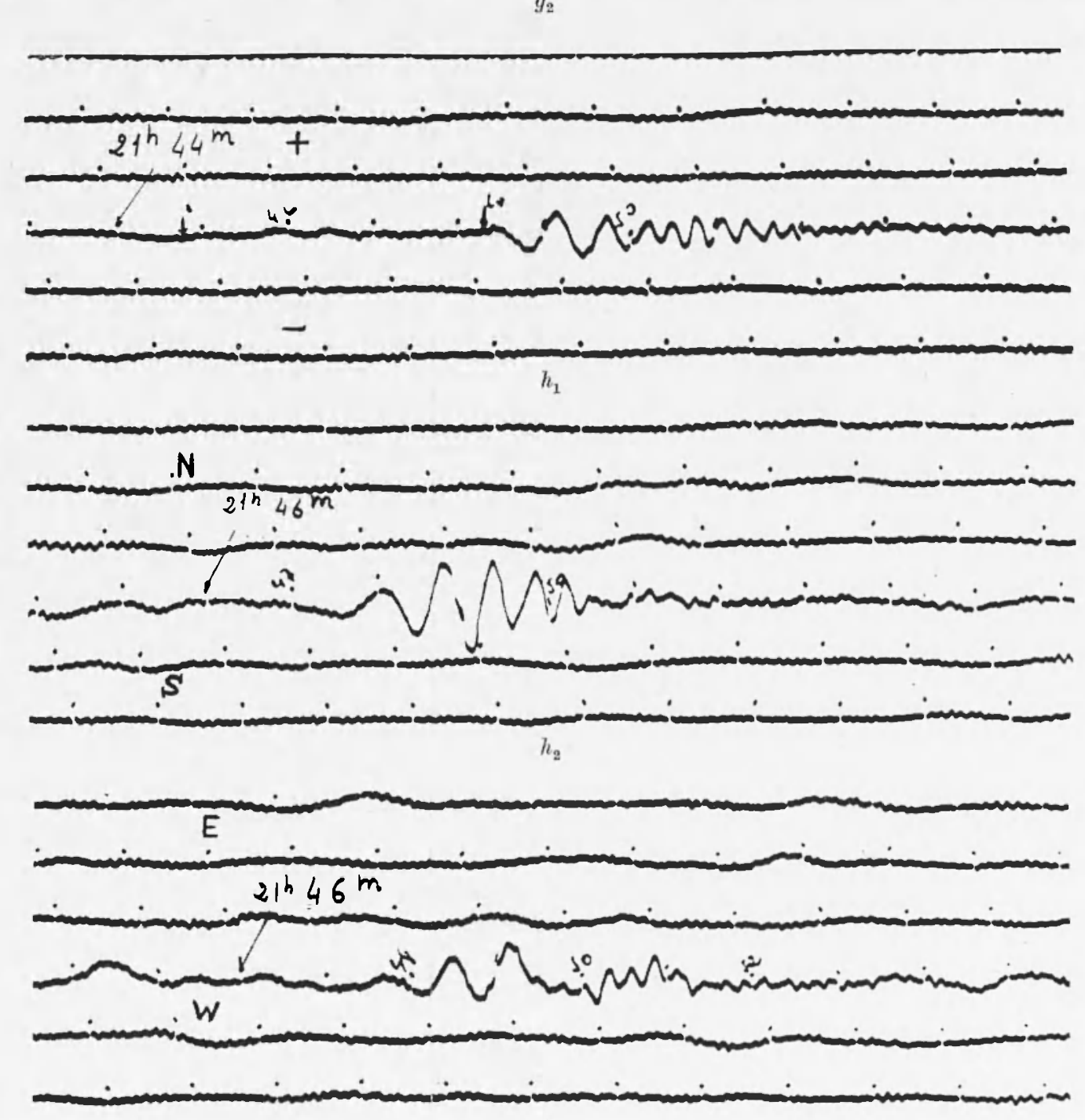


Solo un urto subitaneo, esaurentesi in pochi secondi, può spiegare l'insorgenza di oscillazioni libere nelle stratificazioni della crosta terrestresingolarmente e nel loro insieme -; e solo tali oscillazioni, d'altronde, possono spiegare i lunghi periodi osservati, che mai si riscontrano per così brevi distanze epicentrali — nella registrazione di terremoti, propriamente detti. Al crescere della distanza - a paritì di altre condizioni - figurano onde via via di periodo crescente, non per fenomeno di dispersione (insufficiente a spiegare aumenti così forti per variazioni tanto piccole della distanza), ma per l'entrata in oscillazione di spessori sempre maggiori della crosta. A Toledo (Figg. $2 \tau h_{1}, h_{2}, h_{3}$ ), Uppsala e Unea le oscillazioni registrate interessano, probabilmente, l'intera crosta terrestre.

4. - Fu fatto l'esame delle registrazioni ottenute in numerose stazioni sismiche europee.

Qui mi limito a riportare i risultati relativi alle dromocrone di cinque fasi (tre relative al primo urto; due al secondo).

Le dromocrone, per quanto concerne i tempi, hanno lo zero in corrispondenza di $22^{\mathrm{b}} 41^{\mathrm{m}}$.

Per le longitudinali a lungo periodo (oltre i 20s), per distanze non superiori ai $900 \mathrm{~km}$, vale l'espressione

$$
Y=\frac{1}{6,53 \pm 0,09} X+37^{\mathrm{s}, 4} \pm 1^{\mathrm{s}, 3} \text {. }
$$

dove $Y$ rappresenta i tempi in secondi ed $X$ le distanze in $\mathrm{km}$.

Per le trasversali a medio periodo (da 5 a $9 \mathrm{sec}$ ), valgono per i due impatti, le equazioni:

$$
Y=\frac{1}{\dot{2}, \gamma \pm 0,1 \%} X+24^{s}, 4 \pm \tilde{5}^{s}, 1
$$

ben oltre la diga e che la velocità del fronte d'onda, non era solo legata alla naturale pendenza del fondo, bensi enormemente aumentata dalla spinta del liquido che le precipitava immediatamente a monte (non si dimentichi l'immane estensione della massa liquida), e dal quale veniva letteralmente proietlata verso valle.

Io stesso dicasi per l'attraversamento del Piave, che - sulla base delle registrazioni di Pieve di Cadore - dovrebbe essersi effettuato in $17 \mathrm{~s}$ circa.

In tutte le stazioni sismiche europee, con apparechi a breve periodo, le vibrazioni destate dalla corsa scatenata dell'acqua nella gola del Vajont, accompagnano l'oscillazione con periodo medio di $7^{\mathrm{s}}$ per la durata di $50^{\mathrm{s}}$ circa. 


$$
Y=\frac{1}{2,78 \pm 0,165} X+56 \mathrm{~s}, 1 \pm 7,9
$$

Per le trasversali a lungo periodo (da $20^{\mathrm{s}}$ a $\left.33^{\mathrm{s}} \mathrm{ca}\right)$ :

$1^{0}$ impatto

$$
Y=\frac{1}{3,96 \pm 0,145} X+45^{\mathrm{s}} \pm 6 \mathrm{~s}, 0
$$

$2^{\circ}$ impatto

$$
Y=\frac{1}{3,86 \pm 0,135} X+73^{s, 8} \pm 5^{s, 8}
$$

Le trasversali a medio periodo interessano, come vedremo, lo strato esterno della crosta terrestre. Con riferimento al primo impatto, si può affermare che esso ebbe luogo verso le $22^{\mathrm{h}} 41^{\mathrm{m}} 24^{\mathrm{s}}, 5$. L'errore più probabile di $\pm 5^{\text {s, }} 1$ - piuttosto sensibile — ci permette di arrotondare l'ora origine a $22^{\mathrm{n}} 41^{\mathrm{m}} 30^{\mathrm{s}}$.

Il secondo impatto avvenne $32^{\mathrm{s}}$ dopo, circa.

Le vibrazioni rapide, associate agli scorrimenti interni, di cui si ̀̀ fatto cenno al n. 3, registrate ampiamente a Pieve di Cadore $\left({ }^{*}\right)$, andarono rapidamente smorzandosi con la distanza: ancora presenti a Tolmezzo e a Somplago, a Trieste appaiono registrate, come tracce, solo sugli apparecchi a breve periodo (a fortissimo ingrandimento dinamico). A Padova, dove funzionano strumenti che consentono limitati ingrandimenti dinamici, le perturbazioni legate alle fasi preliminari del grande evento sono pressoché mancanti. A distanze magrgiori, non si ebbe registrazione di onde spaziali, longitudinali e trasversali, nel senso ordinario dei termini. Così a L'Aquila $(4.4 \mathrm{~km})$, dove pure funzionano strumenti a breve

(*) Uno dei motivi che mi hanno indotto a non servirmi delle registrazioni di Pieve di Cadore, ai fini della determinazione dell'energia dell'evento trasformatasi in perturbazione elastica, ì stato appunto la constatazione che le registrazioni di Pieve di Cadore non corrispondono ad onde longitudinali e trasversali propriamente dette e pertanto ad esse non si possono applicare - come è stato fatto da taluno - le formule per la determinazione della magnitudo e dell'energia, di uso corrente in sismologia, Caso mai, dovevano essere assimilate ad onde superficiali (come, del resto, risulta chiaramente dal loro aspetto); anche in questo caso, però, solo con grande riserva (essendo il meccanismo di propagazione sostanzialmente diverso da quello delle onde superficiali tipo Rayleigh e tipo Love) si potrebbero accogliere i risultati del calcolo dell'energia a loro associata. 
periodo di elevatissimo ingrandimento, cosi a Roma ( $\mathrm{km} \mathrm{480),} \mathrm{a} \mathrm{Stoccarda,}$ a Strasburgo, a Messina $(940 \mathrm{~km}$ ), ecc. Sono stati i due impatti (solido e liquido) a provocare la registrazione delle onde a lungo periodo nelle stazioni europee dotate di apparecchi idonei. I moti tellurici ad essi associati, non sono peró paragonabili a terremoti, quali vengono definiti in sismologia. L'energia animante l'enorme massa (solida prima, quindi liquida) in movimento, si è trasferita alla Terra sotto forma di urto continuato, sia pure contenuto entro il limite di pochi secondi; non ha avuto, ad ogni modo, il carattere di immediata subitaneità, proprio delle subitanee rotture dell'equilibrio delle tensioni in zona sismica, cui segue — in campo elastico - la formazione delle onde longitudinali e trasversali ordinarie. Non è nemmeno corretto, pertanto, parlare di onde superficiali tipo Love e Rayleigh, di cui si è fatto cenno in precedenti relazioni. La creazione di queste onde (almeno di quelle a più lungo periodo) richiede la sovrapposizione di coppie di onde longitudinali e trasversali ordinarie, di cui - come si è visto - è pressochè mancata la formazione all'origine. Del resto, dove queste oscillazioni a lungo periodo appaiono regolarmente registrate (Trieste, L'Aquila, Stoccarda, Strasburgo, Uppsala...), esse iniziano come effettive onde longitudinali, seguite (come a Trieste) da onde trasversali-tangenziali, entrambe propagantisi con la velocità delle onde longitudinali e trasversali proprie dello strato del granito (Trieste). Senonché, l'enorme periodo cui sono associate, porterebbe a concludere trattarsi di onde longitudinali e trasversali del mezzo, e non nel mezzo. In altre parole, le onde in questione dovrebbero risultare le oscillazioni proprie delle stratificazioni della crosta terrestre, o di sue parti. Si può in prima approssimazione - come si è detto più sopra - paragonare le oscillazioni a lungo periodo, collegate al movimento tellurico verificatosi al Vajont, alle oscillazioni destate da urto in un piatto indefinito. In questo caso, si puó ora addirittura tentare, partendo dai dati relativi alle onde trasversali, di determinare lo spessore approssimativo degli strati entrati in vibrazione propria sotto l'urto immane delle masse solida e liquida, in caduta libera.

Nella teoria delle onde elastiche progressive in piatti piano-paralleli indefiniti, vale - nel caso di oscillazioni asimmetriche $\left(^{9}\right)$ - la relazione

$$
v=\sqrt{\frac{\pi}{\sqrt{3}} b \sqrt{\frac{2}{1-\sigma}}} \sqrt{\frac{H}{T}},
$$

dove $v$ è la velocità di propagazione dell'onda elastica nel piatto, $b$ la velocità delle onde trasversali proprie del mezzo, $\sigma$ il rapporto di Poisson, 
$H$ lo spessore del piatto indefinito e $T$ il periodo dell'onda. La formula vale per lunghezze d'onda decisamente maggriori di $H / 2$.

Posto

$$
C=\frac{\pi}{\sqrt{3}} b \sqrt{\frac{2}{1-\sigma}},
$$

si ottiene

$$
H=\frac{v^{2} \cdot T}{U}
$$

Per $\sigma=\frac{1}{4}$

$$
C=2,963 b \text {. }
$$

Applichiamo la [1] alle onde trasversali, a lungo periodo, osservate in alcune stazioni europee.

Cominciamo da quelle con periodo proprio variante da $5^{\mathrm{s}}$ a $9^{\mathrm{s}}$. Per esse, fatto $b=2,5 \mathrm{~km} / \mathrm{sec} \mathrm{ca}$, si ottiene

$$
C=7,4 \text {. }
$$

Poiché dalla loro dromocrona risulta $v=2,8 \mathrm{~km} / \mathrm{sec}$ ca, dalla [1] si ha

$$
H=1,06 T .
$$

Scegliendo per $T$ i valori osservati in varie stazioni europee, avremo:

$$
\begin{aligned}
& \text { per } T=5^{\mathrm{s}} \quad(\text { Somplago) } \quad H=5,3 \mathrm{~km} \\
& T=7^{\mathrm{s}} \quad \text { (Coira, Strasburgo) } \quad H=7,4 \text { ” } \\
& T=\tau^{\mathrm{s}}, 5 \text { (Vienna) } \quad H=8,0 \text { ” } \\
& T=8^{\text {s }} \quad \text { (Ravensburg, Heidelberg) } H=8,5 \text { " } \\
& T=8^{\mathrm{s}, 5} \text { (Isola, Collm) } \quad H=\mathbf{9 , 0} \text { ” } \\
& T=9^{\text {s }} \quad \text { (Kagabria, Tübingen, } \\
& \text { Stoccarda, Feldberg, Parigi) } \quad H-9,5 \text { ». }
\end{aligned}
$$

Prescindendo dall'aumento del periodo per dispersione - di lieve entità, in ogni caso - lo spessore dello strato interessato da questa oscillazione varia da 5 a $10 \mathrm{~km}$ ca. Esso può identificarsi con i sedimenti del quaternario e del terziario, di cui si è altra volta provata la pressoché uniforme estensione in tutta Europa $\left({ }^{10}\right)$, naturalmente con spessori variabili da luogo a luogo. 


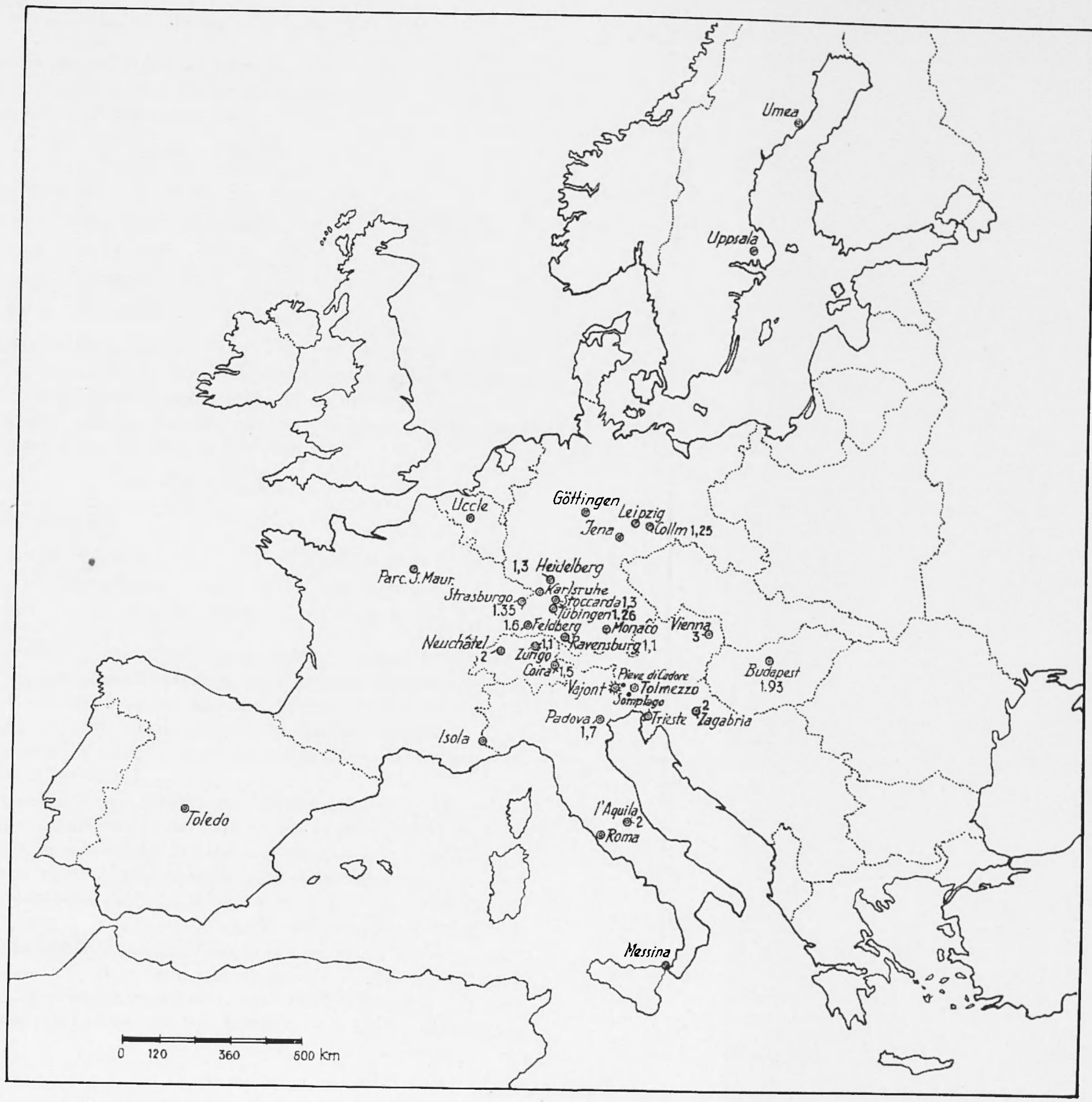

Fig. 28 - Alcune stazioni sismiche europee, presso le quali fu registrato l'evento del Vajont. Il numero posto accanto al nome di parecchie di esse (p. es.: Ravensburg 1,1; Vienna 3, ecc.) sta ad indicare il rapporto fra le ampiezze del movimento provocato dal secondo e dal primo impatto, rispettivamente. 
In alcune stazioni sismiche, appaiono chiari esempi di trasversali con periodi variabili da 12 a 18 sec. Facendo per esse, $b=3 \mathrm{~km} / \mathrm{sec}$, $v=3,5 \mathrm{~km} / \mathrm{sec}$, si ottiene

$$
H=1,376 T
$$

Ne consegue per

$$
\begin{array}{lll}
T=12^{\mathrm{s}} \quad \text { (Neuchâtel, Budapest) } & H=16,5 \mathrm{~km} \\
T=16^{\mathrm{s}} \quad \text { (Uccle) } & H=22 & \text { " } \\
T=16^{\mathrm{s}-18^{\mathrm{s}}} & \text { (Strasburgo) } & H=22-25 \quad \text { } \\
T=18^{\mathrm{s}} \quad \text { (L'Aquila) } & H=25 &
\end{array}
$$

Lo strato di medio spessore pari a $20 \mathrm{~km}$ ca (comprendente il così detto "granito ") risulta universalmente diffuso in tutti i continenti e ad esso compete un'oscillazione libera di 16-18 sec ca ("1).

Se passiamo alle oscillazioni trasversali a lungo periodo, fatto $b=3,6 \mathrm{~km} / \mathrm{sec}$ e $v=3,9 \mathrm{~km} / \mathrm{sec}$, si ottiene

$$
H=1,427 T \text {. }
$$

Sar̀i, pertanto, per

$$
\begin{array}{lll}
T=20^{\mathrm{s}-22 \mathrm{~s}} & \text { (Trieste) } & H=28,5-30 \\
T=30^{\mathrm{s}} & \text { (Strasburgo, Uecle) } & H=43 \mathrm{~km} \\
T=33^{\mathrm{s}} & \text { (Stoccarda, L'Aquila) } & H=47 \mathrm{~N} .
\end{array}
$$

Per Trieste, lo strato interessato dovrebbe contenere il "granito " e l'intermedio, mentre per Strasburgo, Uccle e Stoccarda, dovrebbe trattarsi dell'intera crosta terrestre, fino alla superficie di Mohorovicic.

Per L'Aquila, for'se, soltanto fino all'intermedio compreso.

Ad ogni modo, i valori ottenuti valgono in prima approssimazione, come ordine di grandezza.

Un altro fatto va sottolineato, nei riguardi della propagazione dell'energia associata alle onde trasversali - con periodo dell'ordine di $7 \mathrm{~s}$, in media - provocate dai due impatti, seguitisi con l'intervallo di $32^{\text {s}} \mathrm{ca}$. L'osservazione mostra chiaramente che essa ha avuto una non uniforme distribuzione, nel senso che i due urti rivelano una direzione privilegiata per quanto concerne il massimo d'energia trasmessa; così, mentre il primo urto ha trasferito maggior energia in direzione $N W$ (Strasburgo, Stoccarda, ecc. ...), il secondo ha sprigionato il massimo trasferimento verso $N E$ (Vienna). NellaFig. 28, i numeri che figurano accanto alle stazioni d'osservazione, indicano il rapporto fra le ampiezze ivi provocate 
dal secondo impatto rispetto a quelle relative al primo. La distribuzione dei numeri consente di concludere che il secondo impatto ha sviluppato un'energia circa 4 volte maggiore di quella relativa al primo (il rapporto delle ampiezze può infatti essere calcolato di $2: 1$ ).

La diversa direzione di propagazione del massimo di energia associata ai due urti, può spiegarsi nella diversa modalità che ha presieduto agli stessi: il primo, infatti, si è essenzialmente manifestato come violenta proiezione di un'enorme massa di rocee e detriti da Sud a Nord, dal monte Toc verso il monte Borgà; il secondo, con le caratteristiche di una poderosa mazzata, dall'alto al basso, nelle specie di cirea 20 milioni di $\mathrm{m}^{3}$ d'acqua, precipitati nella forra del Vajont da un'altezza di 400 metri circa.

5. - Stato della roccia d'imposta e del calcestruzzo della diga dopo l'evento.

Un primo esame delle caratteristiche elastiche della roccia d'imposta e della diga del Vajont (Figg. 29) dopo la soluzione del contrasto tettonico, fu eseguito sul posto in condizioni particolarmente difficili, ai primi di Novembre 1963 in collaborazione, come di consueto, con la dott. M. O. Spadea. Di esso fu riferito all'ENEL-SADE.

Per quanto riguarda la roccia indagata in quell'occasione si è riscontrato che, in sinistra, il massiccio roccioso che sovrasta quota 590 (vecchia strada per Erto) aveva conservato le sue caratteristiche elastiche, quali aveva prima del 9 Ottobre $\left(E=3,7.10^{5} \mathrm{~kg} / \mathrm{cm}^{2} \mathrm{ca}\right)$. La parte prossima alla quota coronamento diga, accennava invece ad un flessione nel modulo, conseguente all'enorme pressione sopportata $(E$ dell'ordine di $1,65 \times$ $10^{5} \mathrm{~kg} / \mathrm{cm}^{2}$ ).

In sponda destra fu possibile indagare solo la parte più prossima al coronamento diga, dove pure fu rilevato un decadimento del modulo, anche se lievemente inferiore di quello rilevato in sinistra.

Le indagini estese alla diga condussero a concludere che da una quota prossima a $590 \mathrm{~m}$ fino al coronamento, il modulo aveva un valore medio di $6,2 \times 10^{5} \mathrm{~kg} / \mathrm{cm}^{2}$ (per un valore medio della velocità delle onde longitudinali di cirea $5,2 \mathrm{~km} / \mathrm{sec})$.

Nel tratto più prossimo al coronamento, si è osservata una lieve flessione delle caratteristiche elastiche, la velocità essendo risultata dell'ordine di 4-4,2 km/sec. Nella parte indagata il calcestruzzo presentava quindi un'elevatissima rigidità; l'enorme pressione esercitata dalla spinta dell'acqua in uscita, sembrava non aver provocato danno apprezzabile. 


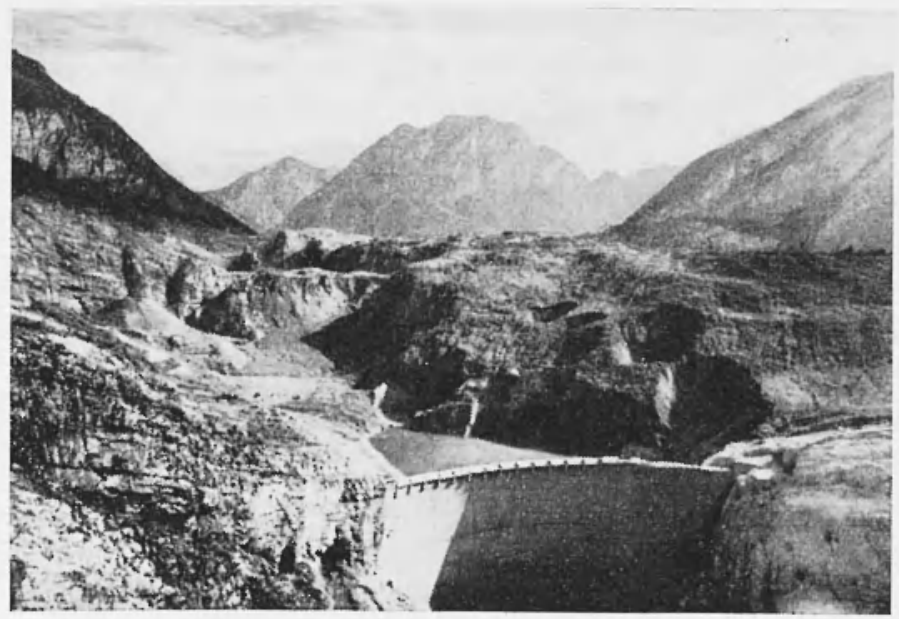

Fig. 29

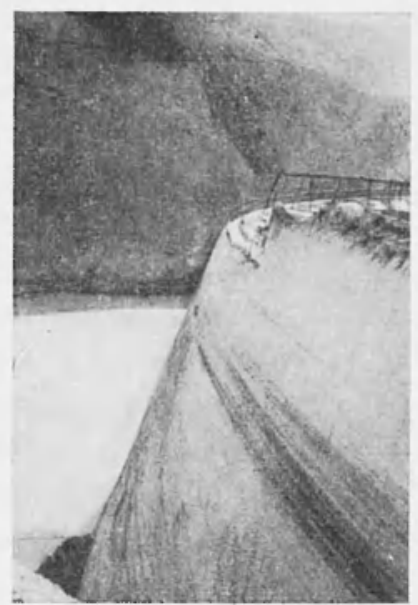

Fig. $29 a$

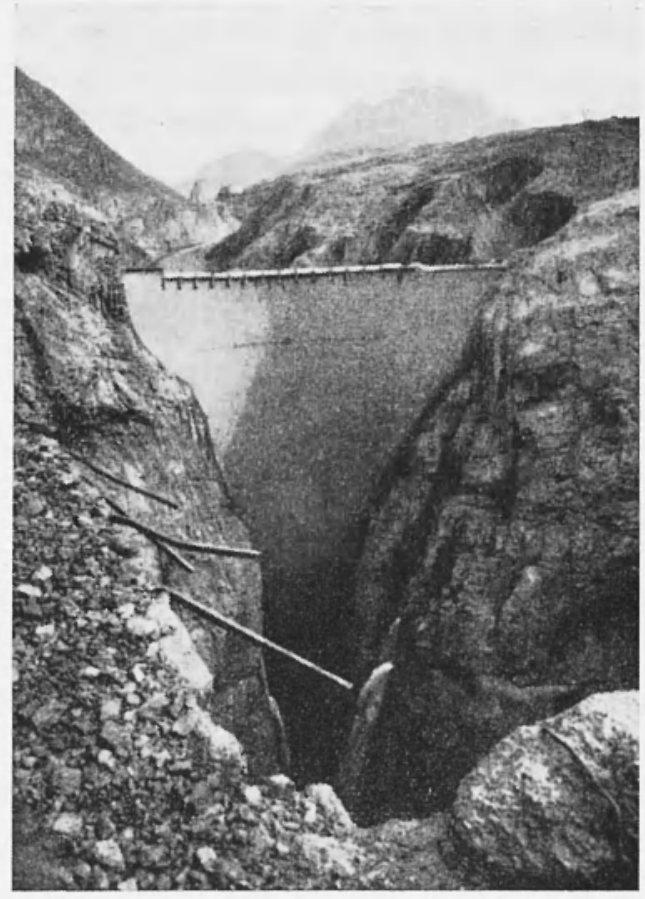

Fig. $29 b$

Fig. 29 - Alcuni aspetti della stretta del Vajont e della diga, subito dopo l'evento del 9 Ottobre 1963 (foto Caloi). 
Inoltre, i vibrogrammi, ampli e netti, ottenuti sulla diga, testimoniavano di un ottimo collegamento roccia d'imposta-diga, lungo i tragitti interessati essendo risultata non apprezzabile la dissipazione di energia elastica.

Poiché, nella zona d'imposta della diga "Carlo Semenzà, all'incirca. fra le quote 650 e 720, in destra e in sinistra, nel 1961 il mezzo (di sensibile omogeneità) presentava un modulo di Young - in entrambe le zone dell'ordine di $2,5 \cdot 10^{5} \mathrm{~kg} / \mathrm{cm}^{2}$ (per velocità delle onde longitudinali aggirantisi, nei tratti in questione, sui $3,30 \mathrm{~km} / \mathrm{sec}$ ), si poteva concludere che l'efïetto del grande urto conseguente all'ultimo cedimento, si era tradotto in un decadimento del modulo elastico nella misura del $34 \%$ circa nell'ammasso roccioso, cui è ancorata la diga nella parte superiore in sinistra, e lievemente inferiore alle corrispondenti alte quote in destra.

Un'indagine più accurata, condotta dal 9 al 13 Dicembre 1963, intorno alla zona d'imposta della diga e sul calcestruzzo della stessa, da quota ponte-canale ( 590 circa) al coronamento, ha sostanzialmente confermato i dati precedentemente ottenuti.

Il modulo elastico della roccia, massimo verso le quote più basse, tendeva a diminuire (come, del resto, normalmente si osserva) ver'so l'alto presentando le sue più accentuate flessioni nelle zone a contatto con la diga, verso il coronamento. Il tratto da quota 590 a quota 720 in spalla destra - non investigato nel precedente intervento - mostrò velocità (e quindi modulo) leggermente superiori a quelle ottenute nell'analogo tragitto in sponda sinistra.

Restava in conclusione confermata la flessione del $34 \%$ circa nei valori del modulo elastico della roccia ottenuti nel 1961, nelle zone dove maggiore si era esercitata la spinta d'uscita dell'ondata.

Per quanto concerne il calcestruzzo, i risultati erano ancora piu confortanti: la risposta elastica delle diga non poteva infatti riuscire migliore. Lungo tutti i tragitti esaminati la velocità risultò ovunque superiore a $4,4 \mathrm{~km} / \mathrm{sec}$, i valori massimi essendosi osservati alle basse quote (dove la velocità delle onde longitudinali doveva ritenersi dell'ordine di $5,7 \mathrm{~km} / \mathrm{sec})$.

Si poteva pertanto asserire che la diga e la roccia d'imposta avevano sostenuto la tremenda prova con il minimo danno, specie per quanto si riferisce allo sbarramento.

Possiamo infine osservare che, in seguito all'urto, la roccia prossima al coronamento era tornata a condizioni elastiche dell'ordine di quelle preesistenti alle iniezioni di cemento. Sembra pertanto che la spinta del- 
l'acqua in uscita abbia riportato il mezzo alle primitive condizioni naturali, quasi avesse provocato il distacco dell'intrusione cementizia dal tessuto roccioso.

\section{6. - Sismicità della zona prossima alla valle del Vajont; manifestazioni più cospicue dal 1962 al 1963.}

Si è già detto che la zona circostante il Vajont presenta una sismicità, di frequenza e intensità crescenti da Nord verso Sud, dove si riscontrano certi sismi, la cui attività ha assunto in passato (anche recente) proporzioni catastrofiche (come al Cansiglio il 18.X.1936).

Negli ultimi anni, tale attività ì stata di modeste proporzioni, essendosi limitata a microscosse, poche delle quali avvertite dalle popolazioni locali, entro raggi molto limitati. Riporto qui di seguito, elencate in ordine cronologico, alcune delle scosse più chiaramente registrate.

Da zona Monte Pramaggiore si ebbe una sensibile manifestazione, registrata specialmente a Pieve di Cadore, il 17 Marzo 1962. Una scossa più sensibile, dalla stessa provenienza, ebbe luogo il 12.VI.1962. Dall'interpretazione della registrazione ottenuta nelle stazioni sismiche di Pieve di Cadore, Vajont e Tolmezzo, si ha:

Pieve di Cadore: $\quad P g \quad 06^{112} 0^{\mathrm{m}} 51^{\mathrm{s}, 5} \quad$ - azimut $\alpha=116^{\circ}, 5 \quad-$

$$
\Delta=12,7 \mathrm{~km} \text { - energia }=9,15 \times 10^{15} \mathrm{erg}-\text { magnitudo } M=3,1 ;
$$

Vajont:

Tolmezzo:

$$
\begin{aligned}
& a=45^{\circ}, \triangle=17 \mathrm{~km} ; \\
& \Lambda=40 \mathrm{~km} .
\end{aligned}
$$

Dal Monte Bivera (Lago di Sauris), il 2.V.1963, a:

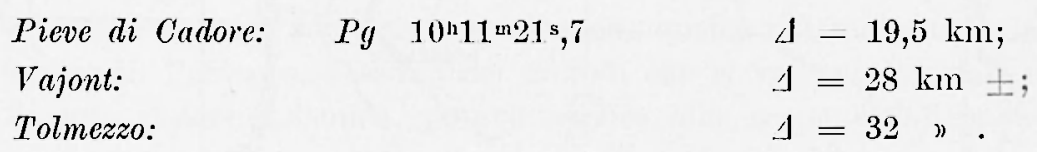

Dal Col Visentin, 1'8.V.1963, una scossa, fortemento sentita anche a Belluno, provocò le seguenti registrazioni:

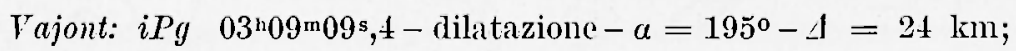

Pieve di Cadore: $P g$ 03 ${ }^{\mathrm{n}} 09^{\mathrm{m}} 12^{\mathrm{s}}, 6$ - dilatazione $\left.\quad\right\rfloor=42$ " ;

Tolmezzo: $P g$ 03 ${ }^{\mathrm{n}} 09^{\mathrm{m} 20 \mathrm{~s}, 5} \mathrm{ca} \quad \Delta=72 \cdots$.

Ie coordinate epicentrali risultarono

$$
\varphi=46^{\circ} 06^{\prime}, 6 \quad N ; \quad \curlywedge=12^{\circ} 15^{\prime}, 2 \quad E ;
$$

l'ora origine

$$
H=03^{\mathrm{n}} 09^{\mathrm{m}} 05^{\mathrm{s}}, 2 .
$$


Il 13.V.1963 al Vajont fu registrata $\left(P g 09^{\mathrm{h}} 5^{\mathrm{m}} 16^{\mathrm{s}}, 3-\Delta=24 \mathrm{~km}\right)$ una leggera replica del precedente.

Dal Col Nudo, il 2 Settembre 1963, provenne la scossa avvertita di V-IV ad Erto, Casso, Longarone, Soverzene, Castellavazzo, Cimolais; fu registrata nelle seguenti quattro stazioni sismiche:

Vajont: $\quad \alpha=137^{\circ}$, $4=6 \quad \mathrm{~km} \mathrm{aar}$;

Pieve di Cadore: $\quad \alpha=155^{\circ}$.

$\Delta=17,5$ " ;

Tolmezzo:

$\Delta=50 \quad$ \% ;

Somplago:

$\Delta=52,5$ ”.

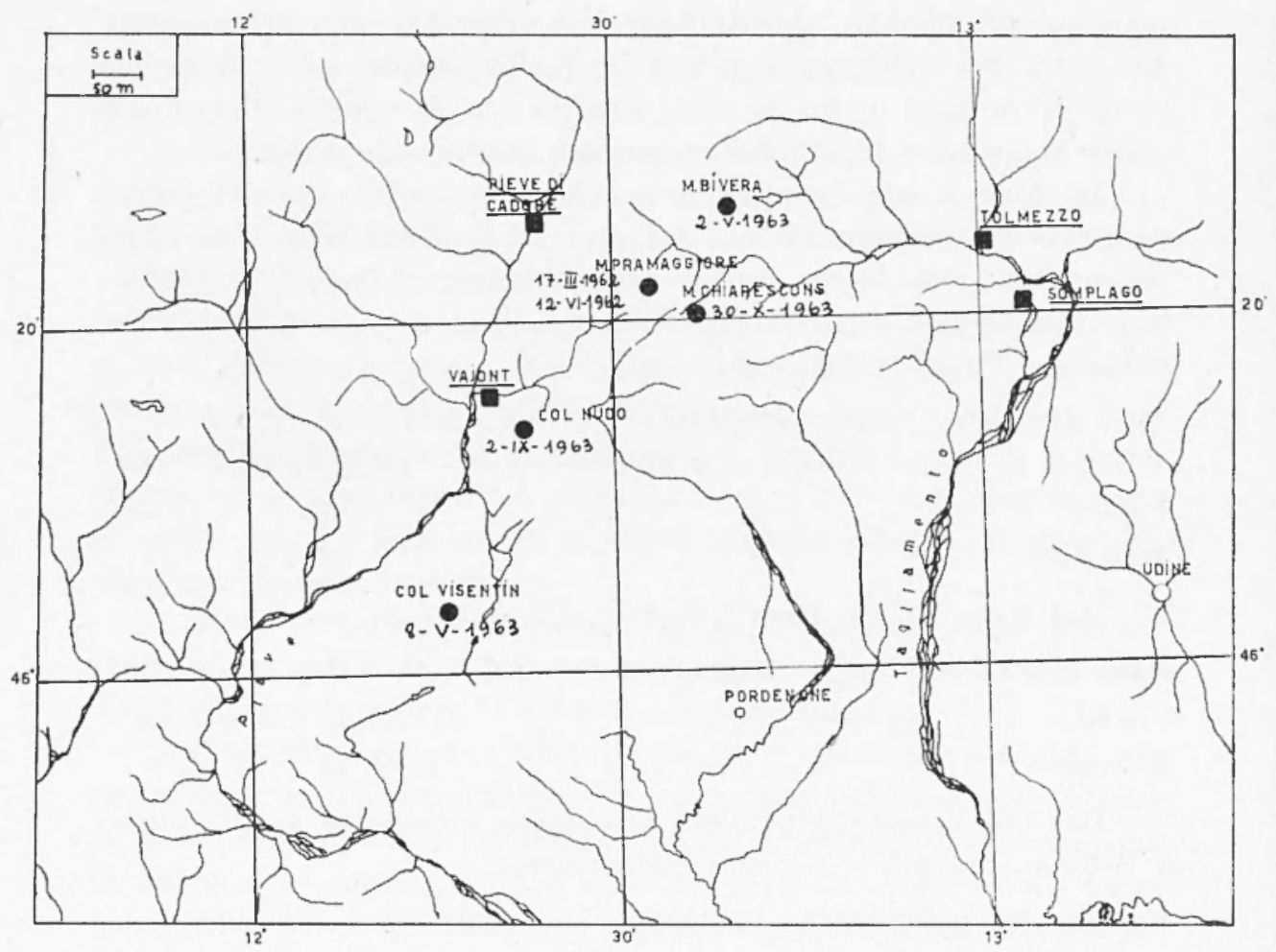

STAZIONI SISMICHE.

SCOSSE in zone limitrofe al vajont

Fig. 30 - Epicentri di alcune delle più notevoli scosse, verificatesi nelle zone limitrofe al Vajont nel 1962-63.

Seguono le coordinate geografiche epicentrali e il tempo origine:
$\varphi=46^{\circ} 14^{\prime}, 7 \quad N ;$
$\lambda=12026^{\prime}, 9 E$;
$H=10^{\mathrm{h}} 18^{\mathrm{m}} 40^{\mathrm{s}, 0}$. 
La sua magnitudo fu $M=2,5$ e sviluppò un'energia dell'ordine di $9,5 \times 10^{14}$ erg.

Di questa scossa si ebbero alcune lievi repliche.

Dal Monte Chiarescons (alta Val Cellina) il 30.X.1963 una scossa di terremoto, chiaramente avvertita dalla popolazione di Cimolais, provocò chiare registrazioni a Pieve di Cadore, ed anche a Tolmezzo e a Somplago:

$\begin{array}{llll}\text { Pieve di Cadore: } & P g & 05^{\mathrm{h}} 06^{\mathrm{m} 21 \mathrm{~s}} & \Delta=19 \mathrm{~km}-\alpha=1220 \\ \text { Tolmezzo: } & & & \Delta=33 " ; \\ \text { Somplago: } & P g \quad 05^{\mathrm{h}} 06^{\mathrm{m} 23^{\mathrm{s}}, 2} & \Delta=39 " .\end{array}$

La sua magnitudo risulto pari a $M=2,75$, avendo sviluppato all'ipocentro un'energia dell'ordine di $2,3 \times 10^{15} \mathrm{erg}$.

Coordinate epicentrali e tempo origine:

$$
\varphi=46^{\circ} 20^{\prime} N ; \quad \lambda=12^{\circ} 34^{\prime} E ; \quad H=05^{\mathrm{h}} 06^{\mathrm{m} 16^{\mathrm{s}}, 5} .
$$

Nella cartina Fig. 30 sono riportati gli epicentri delle scosse sopra citate, alcune delle quali aventi avuto origine presso il bacino del Vajont, senza essere minimamente collegate col contrasto tettonico in atto, a monte della diga, alla base del Monte Toc.

\section{7. - Profondità media approssimativa delle microscosse a monte della diga.}

Ia determinazione della profondità delle microscosse avvenute in sponda sinistra, a monte della diga del Vajont non si presentava facile. Data l'esiguità delle scosse, non si poteva fare affidamento che sulla registrazione di una sola stazione sismica, quella sistemata nella cabina comandi. Pertanto, nessumo dei metodi che si valgono dei dati di più di una stazione sismica, poteva servire allo scopo. D'altronde neppure i metodi che, nelle ordinarie registrazioni per distanze ipocentrali più o meno notevoli, consentono la determinazione della profondità ipocentrale con i dati di una sola stazione (uso degli accoppiamenti $P$ - $p P$, ecc.) potevano essere utilizzati. Je distanze epicentrali erano troppo esigue per poter sperare di trarre qualche vantaggio dalla sismologia ordinaria.

In realtà, solo un limite superiore poteva stabilirsi per le profondità ipocentrali, ed era quello fornito dalle distanze ipocentrali, date dalla formula

$$
\Delta_{h}=\frac{v_{1} v_{2}}{v_{1}-v_{2}}\left(t_{2}-t_{1}\right),
$$


essendo $v_{1}, v_{2}$ le velocità delle onde longitudinali e trasversali, e $t_{1}, t_{2} \mathrm{i}$ tempi di registrazione delle onde longitudinali e trasversali rispettivamente.

Il valore medio delle velocità $v_{1}$ e $v_{2}$ era grià stato calcolato durante le numerose indagini, compiute allo scopo di determinare le caratteristiche elastiche della roccia nella stretta del Vajont. I valori di $t_{1}, t_{2}$ venivano letti sui sismogrammi. Pertanto, la stazione sismica poteva formire, con buona approssimazione, $A_{h}$ che costituisce, al limite, il massimo valore attribuibile alla profondità ipocentrale.

Solo la determinazione dell'angolo d'emergenza poteva dare un valore approssimativo delle profonditi ipocentrali. $A$ questo riguardo, nel lontano 1934, avevo ideato un metodo per la determinazione delle profondità ipocentrali, basato appunto sulla conoscenza di detto angolo ${ }^{(2)}$. Il metodo si sintetizza, sostanzialmente, nella formula.

$$
\begin{aligned}
\cos ^{2} e & =-\frac{1}{4+\frac{\left(1-\varrho_{1}\right)^{2}}{\varrho_{1} \sin ^{2} \vartheta}}\left\{1+\varrho_{1}-c\left(1-\varrho_{1}+2 \varrho_{1} \sin ^{2} \vartheta\right)+\right. \\
& \left.+2 \sqrt{\varrho_{1}} \sqrt{1-c\left(1-\varrho_{1}\right)-c^{2} \varrho_{1} \sin ^{2} \vartheta} \cdot \cos \vartheta\right\},
\end{aligned}
$$

dove $e$ è l'angolo d'emergenza reale delle onde longitudinali dirette; $\vartheta$ è la distanza epicentrale, espressa in angolo;

$$
\varrho_{1}=\left(1-\frac{h}{r_{0}}\right)^{2}
$$

essendo $h$ la profondità ipocentrale ed $r_{0}$ il raggio terrestre e e è una costante che, nel caso delle onde longitudinali dirette, e per l'Europa Centrale, assume il valore

$$
c=7,76582 \text {. }
$$

A suo tempo tradussi la formula sopra riportata in tabelle, nelle quali, sulla base della conoscenza di e, nota la distanza epicentrale, si poteva avere immediatamente il valore di $h$.

Dalla teoria della propagazione delle onde elastiche, si deduce che l'angolo vero d'emeronza è legato a quello apparente è dalla relazione

$$
\sin \bar{e}-1-2\left(\frac{v_{2}}{v_{1}}\right)^{2} \cos ^{2} e
$$

da cui

$$
\cos e=\frac{v_{1}}{v_{2}} \sqrt{\frac{1}{2}(1-\sin \bar{e})}
$$


Generalmente, il rapporto $\sigma$ di Poisson differisce poco da $1 / 4$. In questa ipotesi, com'è noto, $v_{1} / v_{2}$ rale $\sqrt{3}$, per cui

$$
\cos e=\sqrt{\frac{3}{2}(1-\sin e) .}
$$

Volendo raggiungere un maggiore grado d'approssimazione, si può assegnare al rapporto $v_{1} / v_{2}$ il suo vero valore. Ma, nella generalità dei casi, a motivo degli inevitabili errori d'osservazione, la maggior approssimazione raggiunta sarebbe del tutto illusoria.

VAJONT:- STAZIONL SISIICA (q. 725,6 - coronamento diga).

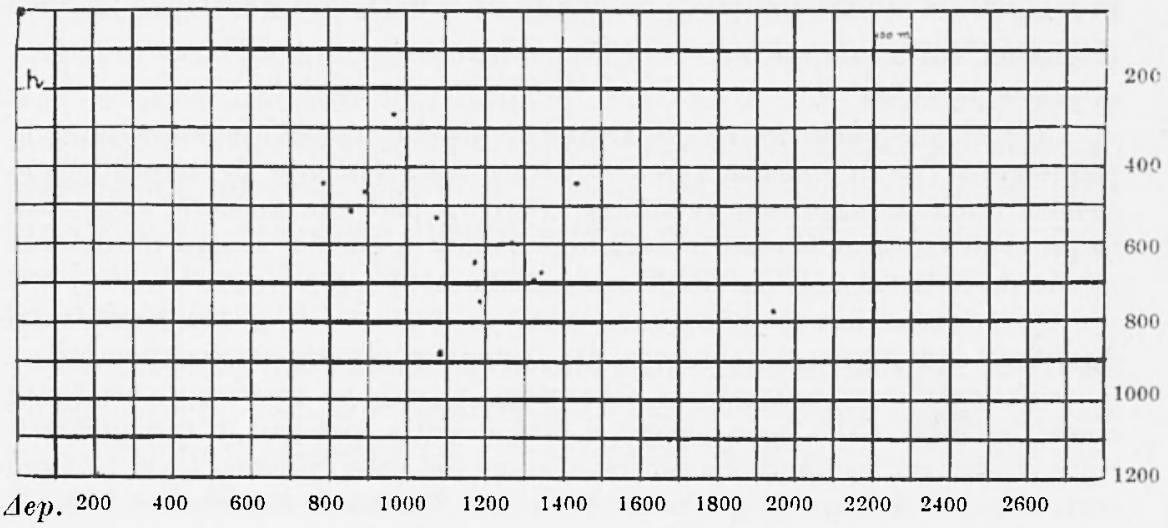

Fig. 31 - Profonditi di alcune microseosse, registrate al Vajont prima dell'evento.

Conosciuto e, la [2] ci consente di calcolare e. D’altronde si ha:

$$
\operatorname{tang} \bar{e}=\frac{x_{Z}}{\sqrt{x_{N}^{2}+x_{E}^{2}}},
$$

essendo $x_{Z}, x_{N}, x_{E}$ gli spostamenti reali corrispondenti al primo impulso dell'onda longitudinale, nelle sue componenti rerticale, verso Ford e verso Hist.

Nel caso specifico, l'uso della [1] è senz'altro da escludere: essa infatti presuppone un tragitto eurvo per il raggio sismico (come in realtà e) e diviene applicabile solo per distanze epicentrali apprezzabili (dellordine di almeno $10 \mathrm{~km}$ ). D'altronde, per distanze ipocentrali infinitesime, come nel caso del Tajont, la propagazione dell'onda longitudinale può 
ritenersi rettilinea $(*)$. Di conseguenza, ottenuto dai sismogrammi $\Delta_{h}$ e l'angolo di emergenza $\bar{e}$, e dalla [2] l'angolo d'emergenza vero, $h$ consegue immediatamente dalla trigonometria elementare.

E ciò che è stato fatto per parecchie microscosse registrate in cabina comandi. Va ancora osservato che, le tre componenti della stazione sismia del Vajont, avevano ingrandimento pressoché uguale: pertanto, l'uso della [3] può prescindere dalla determinazione del valore reale dell'iniziale spostamento del suolo.

La Fig. 31, riporta i valori approssimativi di 14 scosse locali, regristrate presso la cabina comandi, tratte dall'elenco di pag. 31 .

Essi si riferiscono esclusivamente a scossette caratterizzate da dilatazione. Le microscosse contrassegnate da compressione figurano infatti provenire da quote uguali o maggiori di quella della stazione sismica $(725 \mathrm{~m})(* *)$.

$\left(^{*}\right)$ Si può obiettare che lo strato di terreno incoerente sovrastante le pendici del Toe in sinistra, avrebhe dovuto notevolmente deviare il raggio sismico dalla propagazione rettilinea. Non va però dimenticato che, data la provenienza delle scosse, il tragitto dell'onda sismica avveniva totalmente in roccia viva, secondo la traiettoria rettilinea.

(**) L'esistenza di una lunga serie di ipocentri (Fig. 26), quasi tutti distribuiti sullo stesso allineamento, per l'estensione di un paio di chilometri ipocentri tutti caratterizzati da dilatazione - non va trascurata né, tanto meno, innorata, come sono portati a fare alcuni a sostegno di tesi precostituite. Tanto più se questi ipocentri - come nel caso specifico - si trovano tutti a quote inferiori a quelle del fondo del bacino, a sinistra del Vajont, nella zona che ha fatto da base all'ultimo cedimento.

Può dlarsi benissimo che i sondaggi diretti, in corso di esecuzione, abbiano ad indicare, "grosso modo", l'esistenza di una pseudo superficie di slittamento, separante materiale frantumato da altro cosi detto "in sito ". Prevedo, anzi, che si giungerà a questa conclusione. Quel "grosso modo " però toglierà ogni valore probante ad una simile conclusione: l'esistenza di quelle microscosse per cedimento sta a provare che ci fu una lenta "flessione" sul fondo. Gli strati investiti da detta flessione (la cui entità pud essere slata anche di qualche metro sollanto) non è detto si siano frantumati: le loro lacerazioni possono benissimo essersi verificate per grossi elementi, fatto questo che il sondaggio diretto ben difficilmente potrebbe dirimere.

Quei pochi metri di cedimento sul fondo (che un sondaggio diretto, forzatamente a grandi maglie, non potrà mai precisare) furono sufficienti, alla fine, a provocare il risucchio del materiale soprastante Iungo le pendici del Toc, determinando - ad un certo punto - un vero e proprio fenomeno di proiezione di tutta la massa incoerente in lentissimo movimento, dalla sponda sinistra alla sponda destra del Vajont.

Solo in questo modo - partendo, del resto, dai dati di fatto sopra citati - può chiaramente essere spiegato un fenomeno " osì unilario, improvviso, 


\section{8. - Conclusioni.}

L'allucinante catastrofe del 9 Ottobre 1963 si rivela con caratteri talmente inusitati da escludere ogni possibilità di previsione, almeno sulla base delle conoscenze disponibili ante factum. A posteriori, sulla base delle esperienze geodinamiche sopra riassunte e della visione della massa franata, la successione degli avvenimenti può essere cosi sintetizzata.

I lavori di sbancamento trovano la zona in una instabilità dinamica solo assopita, instabilità associata ad una microsismicità frequente in regioni dove intensa è stata, nelle ultime epoche geologiche, l'azione dell'orogenesi.

Le esplosioni iniziali - necessarie per i lavori - riattivano il processo tettonico, in precario equilibrio. La inquietudine clinografica, sorpresa in atto nell'autunno 1958, ne è una testimonianza: le due sponde della stretta del "Vajont " risultano animate da probabili ondulazioni torcenti, quella di sinistra con tendenza verso la zona instabile. Nel Settcmbre 1959 il sistema sembra aver raggiunto nuove posizioni di equilibrio, quasi come un ritorno alla normalità (v. relazione 1960).

La latente microsismicità locale viene successivamente riattivata (Maggio 1960); essa si intensifica nell'Ottobre-Novembre 1960; ad essa va attribuito il probabile frantumamento di setti rocciosi, ancora resistenti in sponda sinistra alla fine del 1959 .

Inizialmente, la microsismicità agisce sul fondo (mascherato dai sovrastanti materiali di frana) di una sorta di "micro-geosinclinale", esistente fra la sponda sinistra del Vajont e le pendici del Toc. Le registrazioni della stazione sismica, infatti, fino ai primi mesi del 1962, sono quasi tutte caratterizzate da dilatazioni, provenienti dalla stessa stretta striscia, stendentesi ad un azimut di $110^{\circ}$ circa dalla stazione sismica, per una lunghezza di due chilometri e mezzo circa. Successivamente, prevalgono le compressioni, associate a scosse tutte provenienti dall'interno del Toc, a sud della zona in fase di cedimento: scosse che possono essere attribuite ad un residuo di orogenesi.

velocissimo". E solo un fenomeno unitario, improvviso, velocissimo - come quello che ha interessato la sponda sinistra del Vajont sul fronte di oltre due chilometri - può, a sua volta, spiegare l'istantanea fuoruscita di un'immane ondata di oltre 20 milioni di metri cubi d'acqua, bruscamente proiettata ad oltre 400 metri di altezza. 
E da ritenere che, nella zona, da me definita come "micro-geosinclinale ", si sia verificato un difetto di massa (“interstizio " fra due monti collaterali?): è verso questa zona che, ai primi di Ottobre, si appresta ad essere risucchiato il materiale sovrastante. Alcuni minuti prima dell'ultimo cerlimento, il materiale compresso contro il Toc comincia, dal fondo, a scorrere lungo la montagna: solo in questo modo può infatti spiegarsi il dispiegamento di energia elastica, sufficiente a determinare la registrazione di movimenti tellurici disordinati già verso le $22^{\mathrm{n}} 38^{\mathrm{m}}$ a Pieve di Cadore $(17 \mathrm{~km})$, a Tolmezzo $(55 \mathrm{~km})$ e a Somplago $(53 \mathrm{~km})$. Movimenti franosi di superficie infatti, verificantisi sopra materiale già sconnesso, elasticamente inerte, non avrebbero determinato movimenti tellurici, registrabili a tanta distanza.

Il materiale in discesa accentua la sua pressione sui setti di roccia, ancora resistenti in sinistra del Vajont. Questi cedono bruscamente verso le $22^{\mathrm{n}} 41^{\mathrm{m}} 30^{\mathrm{s}}$ : assieme ad essi, tutta la massa fino ad allora trattenuta viene letteralmente proiettata verso la sponda destra del Vajont. Da questo urto originano onde di flessione, a lungo periodo, registrate negli strumenti a lungo periodo, funzionanti in Europa. L'acqua (20 milioni $\mathrm{di}^{3} \mathrm{ca}$ ) viene schizzata fuori dall'alveo, fino ad oltre 400 metri di altezza. La sua successiva caduta nella forra del Vajont (32s ca dopo il primo urto), a valle della diga, provoca altre onde di flessione della crosta terrestre, chiaramente registrate a Trieste, a Coira, Strasburgo, Vienna, Budapest, L'Aquila, Parigi, Bruxelles, Uppsala, Toledo, ecc.

Questo meccanismo per l'evento del Vajont - così a posteriori, ricostruito, anche nella sua fase conclusiva, - spiega fra l'altro, come la diga abbia potuto reggere alla pressione delle acque in uscita che, nei suoi riguardi, fu essenzialmente di contenimento laterale. 


\section{APPENDICE}

1. - La nuova stazione sismica del Vajont (Figg. 32) ha iniziato regolare funzionamento nel Maggio 1964. Fino all'Agosto 1965 l'attività sismica da essa registrata, fatta astrazione di quella derivante dall'ordinaria sismicità della Terra, si è limitata a microscosse interessanti settori esterni a quello in cui si verifico l'evento del 9 Ottobre 1963. In particolare, l'attività sismica locale si è mostrata frequente nel settore di NW, lungo una linea di inquietudine che, dalla stazione sismica, è diretta verso Castellavazzo. Le microscosse provenienti da detta linea hanno rivelato l'esistenza di un contrasto tettonico, probabilmente associato ad una nascosta linea di frattura avente all'incirca la stessa direzione. Si è trattato, comunque, di un'attività di lieve entità: le microscosse, infatti, furono di esclusivo carattere strumentale.

A cominciare dal 2 Settembre 1965, e per la durata di alcuni giorni, si è verificato un fatto nuovo: il brusco risveglio del settore sud orientale, dove - per distanze dalla stazione sismica di $3 \mathrm{~km}$ al massimo - centinaia di piccole scosse, non tutte di carattere strumentale (alcune, infatti, sono state localmente avvertite) hanno tenuto in movimento la zona.

L'aspetto delle registrazioni, l'azimut di provenienza delle scosse, la loro intensità media, la natura dell'inizio delle onde longitudinali (quasi tutte dilatazioni), non lasciavano dubbi interpretativi: testimoniavano cioè della ripresa del contrasto tettonico in sponda sinistra del Vajont, che la stazione sismica aveva rivelato esistente, nelle sue registrazioni, dal 1960 al 1963.

Allo scopo di istituire un agevole confronto, sono state riportate nella planimetria della zona (Fig. 26), accanto agli epicentri delle principali microscosse del periodo Maggio 1960-Settembre 1963, quelli di alcune delle più intense microscosse registrate ai primi di Settembre 1965. L'azimut è stato calcolato sulla base degli spostamenti iniziali dei singoli movimenti: il suo valore può ritenersi approssimato entro $\pm 5^{\circ}$.

ì in corso un esame dettagliato delle registrazioni, i cui risultati saranno esposti in una prossima relazione. Fin d'ora però possiamo affermare che il complesso delle dilatazioni viene a cadere in una zona che praticamente coincide con quella in cui si verificarono i cedimenti che precedettero l'evento del 9 Ottobre 1963. Il contrasto tettonico, a suo tempo rivelato, ha avuto quindi una sensibile ripresa nella prima decade del mese di Settembre 1965. Le registrazioni di cui stiamo par- 
lando non si riferiscono - non mi stancherò mai di sottolinearlo - a movimenti franosi, bensi a schianti verificatisi in roccia sana, al di sotto, quindi, della massa informe precipitata nella valle del Vajont nell'Ottobre 1963; al di sotto e, praticamente, lungo la stessa striscia in cui si verifi-

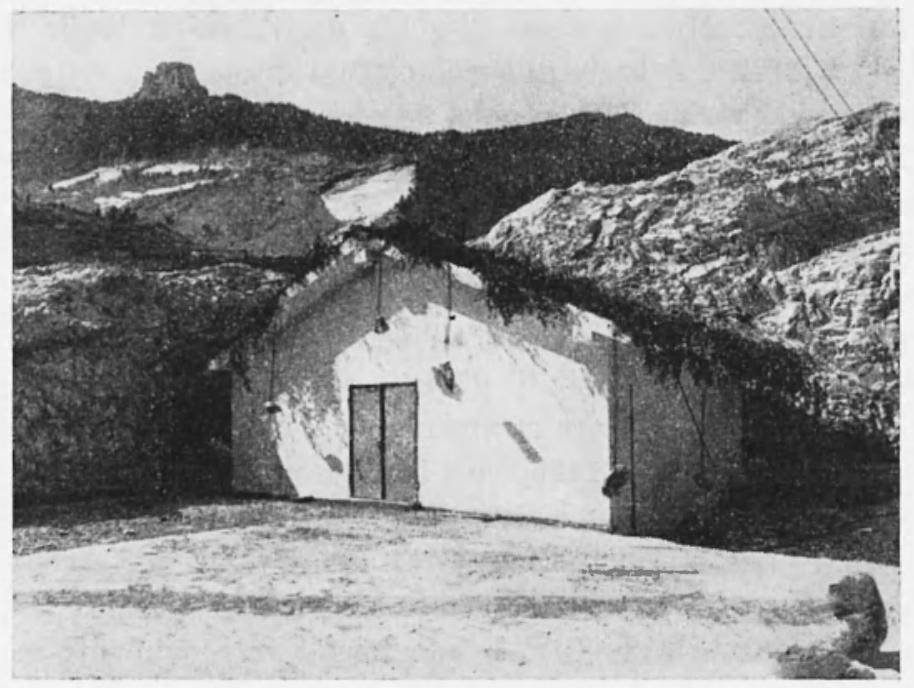

Fig. 32

Fig. 32a

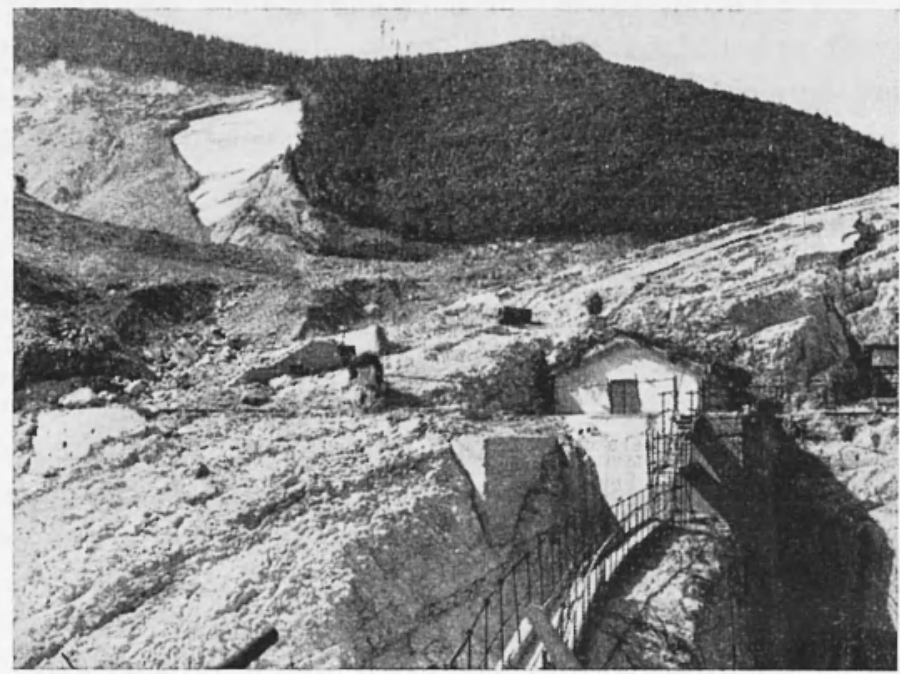

Fig. 32 - La nuova stazione sismica del Vajont, presso la diga "Carlo Semenza". 


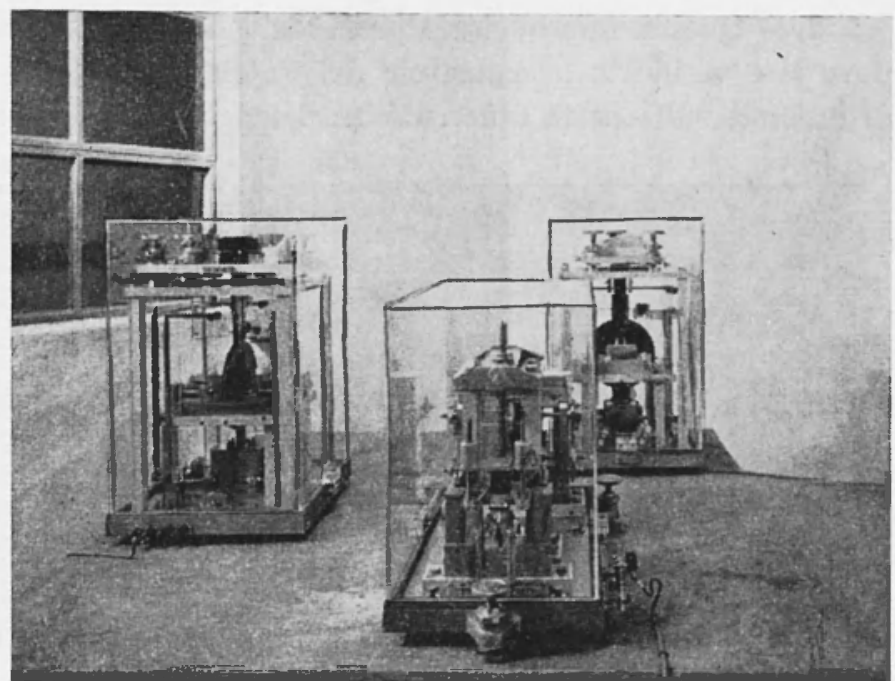

Fig. $32 b$

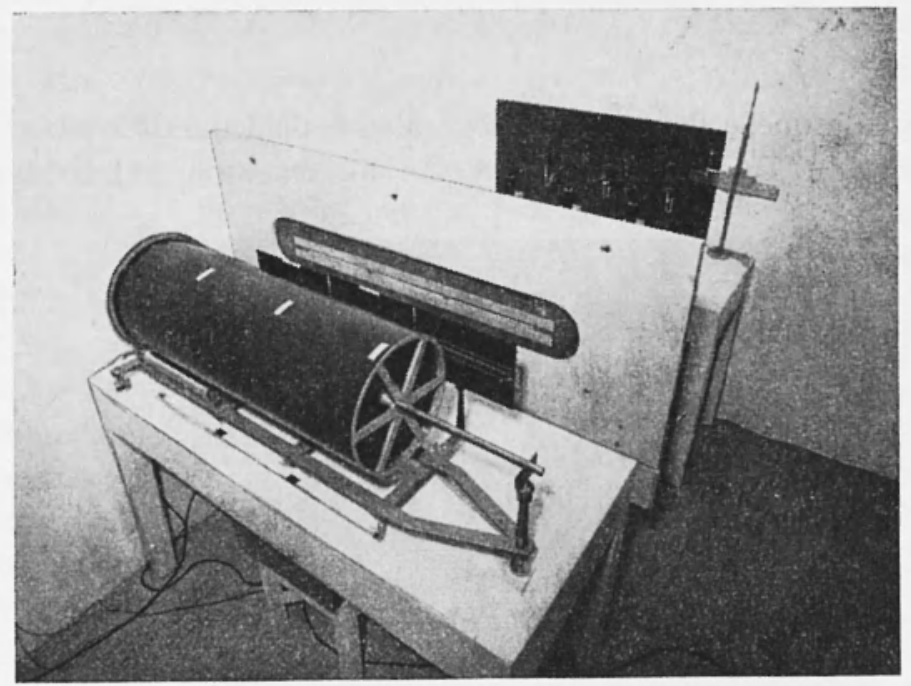

Fig. 32c

carono nel periodo 1960-1963. (Caso mai, c'è stato un lieve trasferimento verso l'interno delle pendici del Toc). Ciò sta a provare - ancora una volta - che le cause dell'evento dell'Ottobre 1963 non vanno ricercate al di sopra della così detta superficie di scorrimento, ma - come ebbi ad affermare in mie precedenti relazioni - in un contrasto tettonico che ha la sua sede dinamica nell'interno del monte.

T)al 10 Settembre la zona è tornata tranquilla. 
2. - Questa improvvisa ripresa delle scosse sismiche, nella zona dove si è verificata la catastrofe del Vajont, mi fornisce l'opportunità di insistere sulla netta differenza fra le registrazioni sismografiche pro-

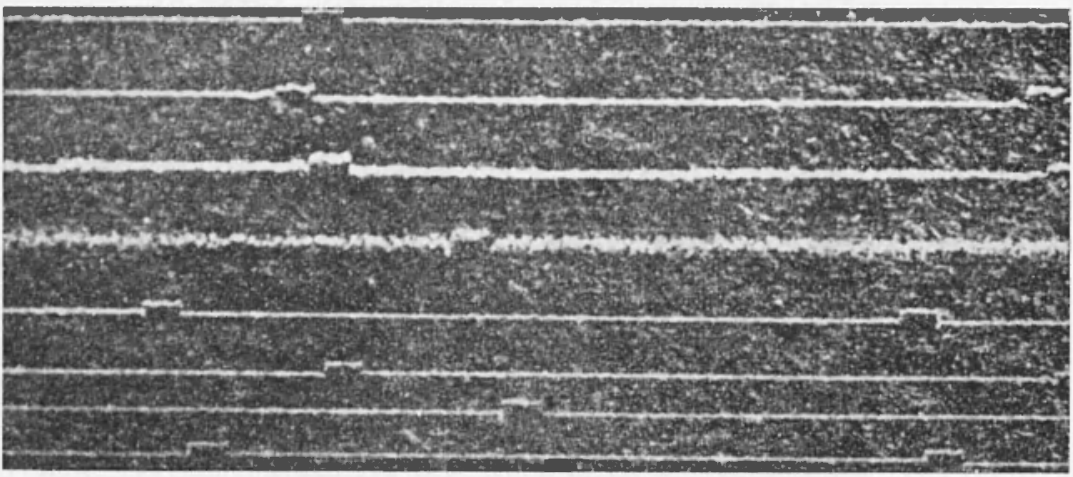

Figr. 33 - Stazione sismica di Oropa: registrazioni di rapidissime vibrazioni destate nel terreno circostante, dal torrente Oropa in piena (Comp. EW Wiechert - 25 Settembre 1956).

vocate da frane e quelle dovute a brusca rottura dell'equilibrio elastico in roccia sana. Quest'ultima, provocata da frattura, improvviso

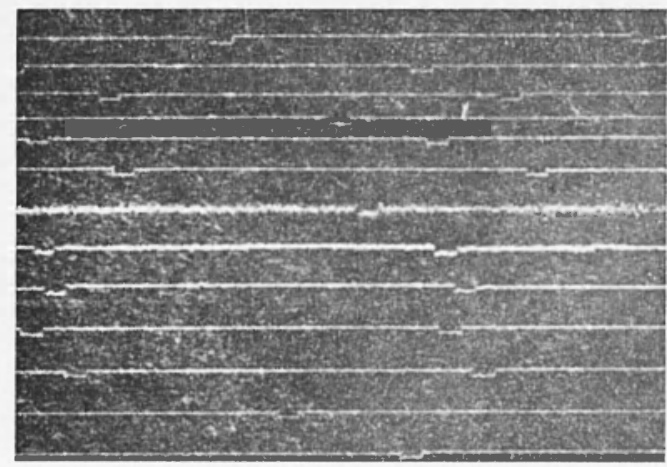

Fig. 34 - Tratto di registrazione del fenomeno, di cui alla Fig. 33, sulla comp. NS Wiechert $200 \mathrm{~kg}$.

schianto per decompressione o implosione, genera onde longitudinali e trasversali ordinarie, seguite eventualmente da onde superficiali (di Love e di Rayleigh). 
Un corpo, fluido - o comunque non consolidato, incoerente, - che scorra violentemente sopra un mezzo solido, genera esclusivamente vibrazioni di carattere superficiale, sotto forma di oscillazioni libere degli elementi di cui il mezzo stesso è composto. Si è già detto del caso delle vibrazioni di Love provocate dal magma che scorra, con violenza, lungo un condotto craterico, in fase esplosiva. Una cosa analoga arviene anche nel caso dei liquidi. Si veda l'esempio delle Figg. 33, 34 che riproducono tratti di una registrazione ottenuta da un sismografo Wiechert $200 \mathrm{kgr}$ presso il Santuario di Oropa dal 25 al 26 Settembre 1956. In seguito ad un'improvvisa alluvione sulle montagne intorno, il torrente Oropa, che scorre a qualche diecina di metri dalla stazione sismica, si gonfiò bruscamente, trascinando gran copia di acqua a valle. L'impeto delle acque, in veloce scorrimento sul fondo e lungo le sponde del torrente, determinò la formazione di vibrazioni rapidissime, che tennero agitati i sismografi per qualche ora, con particolare violenza fra le $20^{\mathrm{h}} 30^{\mathrm{m}} \mathrm{e}$ le $21^{\mathrm{h}} 30^{\mathrm{m}}$ circa del 25 Settembre. E quello che, in proporzioni ben maggiori, è avvenuto durante la corsa delle acque, nella forra del Vajont, dopo essere state scaraventate oltre la diga. La registrazione ottenuta a Pieve di Cadore consiste di oscillazioni a maggior periodo di quelle osservate ad Oropa: ciò perché, data la maggior distanza (17 km in luogo di poche diecine di metri), il mezzo ha, nel frattempo, assorbito le vibrazioni a più elevata frequenza ed ha allungato il periodo di quelle rimaste, per fenomeno di dispersione.

Anche le scosse registrate nella prima settimana del Settembre 1965, sono quindi dovute a rotture nell'ambito della roccia sana; rotture avvenute naturalmente al di sotto della massa incoerente, formatasi dopo l'evento del 9 Ottobre 1963. Anzi, la presenza di questa massa sovrastante è testimoniata da una particolarità delle registrazioni delle scosse più intense, associate a dilatazioni: il brusco cedimento sul fondo, induce un pronto richiamo della coltre sovrastante, che risponde con il suo periodo proprio, come prova la Fig. 35, dove l'inizio del movimento è seguito da un'ampia oscillazione, avente un periodo di $5^{\mathrm{s}}$ cal che costituisce appunto la risposta della coltre incoerente sorrastante. Analogamente per le Figg. 36, 37.

La causa di queste scosse va attribuita all'improvvisa crescita del lago di fronte a Erto, il cui livello, in seguito alle forti piogge del 2 Settembre, era aumentato di 6 metri ca.

Che questa sia stata la causa effettiva è provato dalle scosse sismiche registrate a Tolmezzo, negli stessi giomi e in analoghe circostanze. La brusca piena del Tagliamento, provocata dalle piogge suddette, determinò un aumento di pressione che - in una zona ad elevata sismicità 


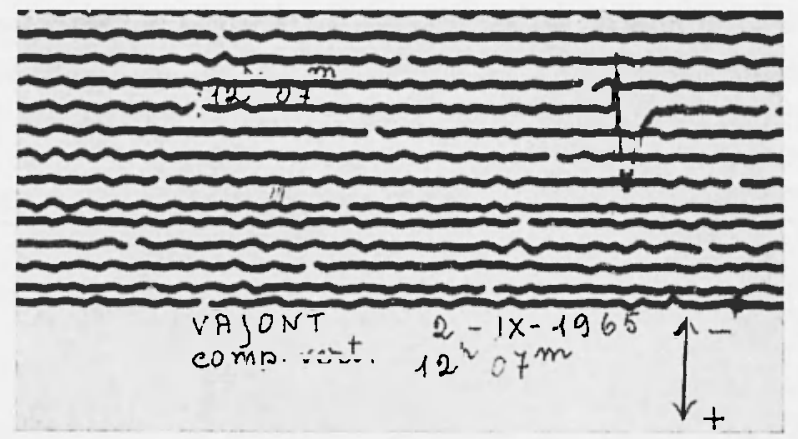

Fig. 35 - Componente verticale di una delle numerose scosse, verificatesi al Vajont nella brusca ripresa dellattivita sismica in zona frana, nella $1^{\text {a }}$ decade del Settembre 1965 .

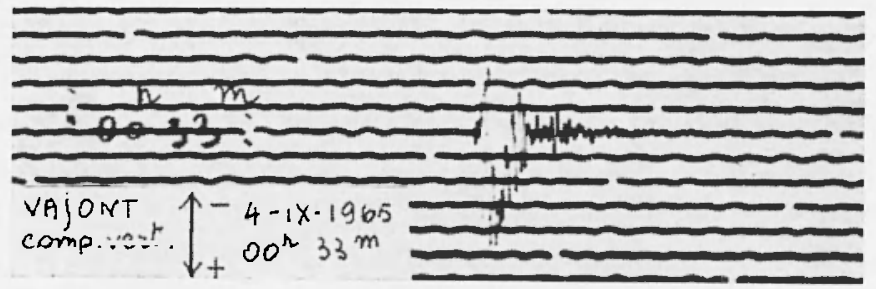

Fig. 36 - Altro esempio, come da Fig. 35.

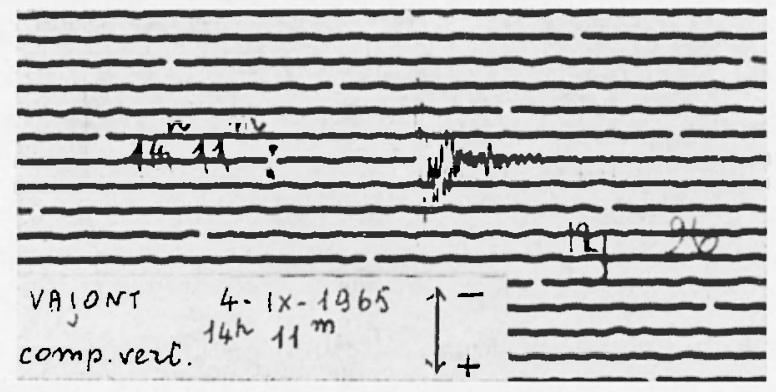

Fig. 37 - Come da precedenti Figg. 35 e 36 .

latente, come è quella di Tolmezzo - è stata sufficiente a determinaro cedimenti sul fondo alluvionale della valle. Le scosse si sono ivi presentate con le stesse caratteristiche di quelle avute al Vajont in zona frana: 
dilatazioni, ciò̀, seguite da ampie oscillazioni, associate a periodo relativamente lungo; oscillazioni che costituiscono la risposta della spessa coltre alluvionale al cedimento sul fondo (Figg. 38, 39).

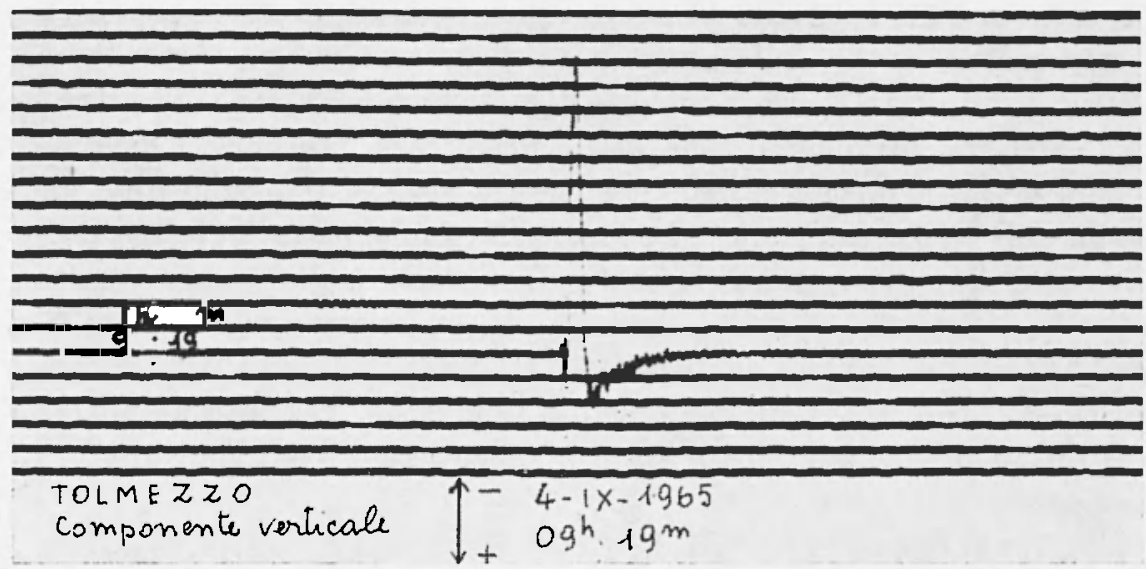

Fig. 38 - Scossa registrata a Tolmezzo, durante la piena del Tagliamento dei primi di settembre 1965. I a spessa coltre fluviale, risponde con il suo periodo alla sollecitazione elastica del fondo roccioso.

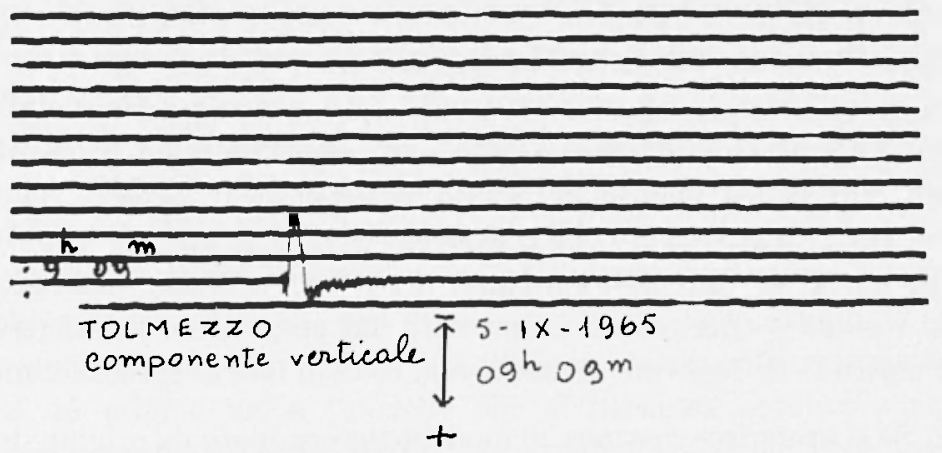

Fir. 39 - Altra scossa a Tolmezzo, come da Fig. 38.

3. - Questa improvvisa ripresa dell'attività sismica sulla sinistra del Vajont, a monte della diga, mi offre lo spunto per tornare un momento sull'interpretazione del loro mecanismo-origine.

L'interpretazione dei movimenti a monte della diga del Vajont, tra la sponda sinistra e il Too, è stata da me stesa, nelle relazioni a suo 
tempo inviate alla SADE, dopo accurato esame dei possibili modelliorigine delle microscosse.

Sono giunto alla conclusione che l'origine a frattura poteva eventualmente essere attribuita alle varie scosse originanti in destra del Vajont, di dove le onde longitudinali giungevano con segni di dilatazione e di compressione, anche dalla stessa posizione, senza un'apparente distribuzione geometrica, come se mutasse di volta in volta il piano di frattura.

Non cosi per quanto concerne le microscosse provenienti dalla sinistra del Vajont. Qui la delimitazione fra le aree, contraddistinte dai segni - e +, è netta; e tale si è delineata fin dall'inizio delle registrazioni sismiche. Dapprima, esclusivamente dilatazioni (-) da una stretta striscia prolungata, compresa entro linee (con origine nella stazione sismica) formanti fra loro un angolo di una diecina di gradi. A meridione di questa striscia, a ventaglio lnngo le pendici del Toc, si sono venute distribuendo successivamente tutte le microscosse, contraddistinte da compressioni $(+)$.

L'interpretazione, quindi, non consentiva dubbi, specie se si tien conto di quella che era la situazione locale.

$\mathbf{E}$ non poteva non articolarsi in queste due sole possibilità:

$\left.1^{\circ}\right)$ Se si ammette il modello associato ad un'origine unica - Fig. $40-$ (cedimento, associato a schianto di roccia per sovraccarico di tensioni, con registrazione di dilatazioni entro un raggio determinato; schianto di roccia per tensioni di spinta verso l'esterno, superanti il limite di rottura, caratterizzato dalla registrazione di compressioni entro un dato raggio), veniva spontaneo attribuire alla zona contraddistinta dalle dilatazioni un moto di sprofondamento, accompagnato da schianti improvvisi. Altrettanto spontaneo risultava associare la zona a ventaglio lungo il Toc, caratterizzato da compressioni a schianti per improvvise decompressioni, quasi per un residuo di orogenesi ("Restspannungen " tensioni residue - dei geologi), agevolato dal progressivo infrangersi dei vincoli elastici che legavano il monte alla zona in fase di sprofondamento.

$2^{\circ}$ ) Se si preferisce ricorrere al modello determinato da origine doppia - Fig. 41 - (scorrimento di strati in sprofondamento lungo una parete stabile, con formazione di una coppia di forze tese l'una verso il basso, l'altra, uguale e contraria, in senso opposto), allora avremo dilatazioni dalla parte dove il cedimento $\grave{e}$ in atto, compressioni dalla parte opposta: questo però se la zona in cedimento è al $d i$ sotto del livello della stazione sismica. Se essa soviasta la stazione sismica, i segni manifestamente s'invertono. 
Nel caso in esame, in questa seconda ipotesi tutte le dilatazioni ad est della stazione sismica sono senz'altro da attribuire a cedimenti lungo le pendici del Toc — in profondità - verso la stazione stessa.

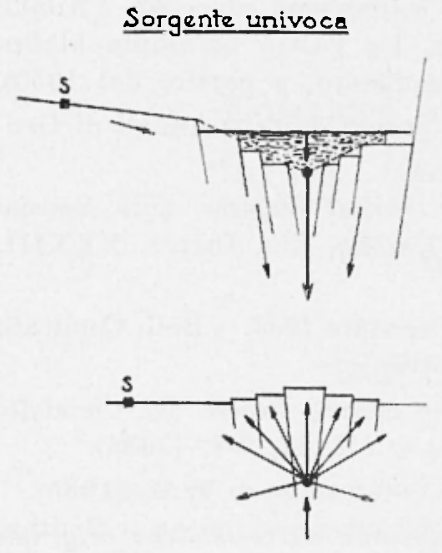

Fig. 40-Modello per sorgente singola di moto sismico (implosione, esplosione).

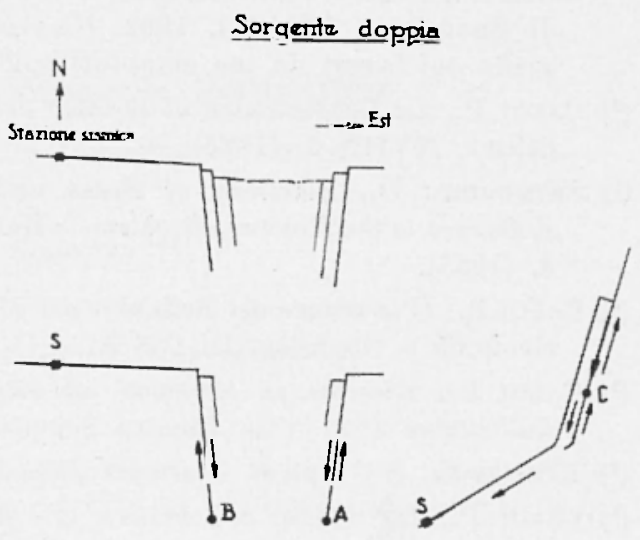

Fig. 41 - Modello a frattura.

Le compressioni fra detta zona e la stazione sismica sarebbero ancora da attribuire a cedimenti - in profondità - verso il centro della zona in flessione. Le altre compressioni a ventaglio lungo le falde del Toc - se a quota superiore di quella della stazione sismica - sarebbero ancora testimonianza di cedimento verso il basso, per la ragione prima detta.

Nell'un caso come nell'altro, la conclusione è sempre la stessa: flessione sul fondo della zona limitata dalla linea punteggiata e cedimento verso di essa della zona sovrastante.

Io, nelle mie relazioni, ho messo l'accento sul primo modello, sia perché più semplice, sia per l'esperienza fatta nello studio di parecchi terremoti appenninici e della Val Padana, facilmente spiegabili con la causa ad origine unica (modello che si riscontra sovente anche negli studi di terremoti giapponesi) $\left({ }^{*}\right)$. Ma anche la soluzione alternativa rientra nello stesso principio: nella prima versione le compressioni nascono - a distanza di tempo - dai cedimenti come da causa ad effetto, nella seconda versione la dilatazione provoca contemporaneamente la compressione nello strato lungo cui avviene l'improvviso scorrimento.

(*) Recentemente, l'esistenza di tale modello d̀ stata confermata anche da Hugo Benioff (14). 


\section{BIBLIOGRAFIA}

(1) Calor P., Aspetti della dinamica di rocce, calcestruzzo ed acque. "Annali di Geofisica ", XV, 2-3, 1962. (Contiene, fra l'altro un'ampia biblio. grafia sui lavori da me compiuti sull'argomento, a partire dal 1950).

(2) Calor P., La Geodinamica al servizio delle grandi dighe, "Annali di Geo. fisica ", XVIII, 1, (1965).

(') Shimozuro D., Elasticity of Rocks under Initial Stresses, with Special Reference to the Fracture Problem. "Bull. Earthq. Res. Inst. ", XXXIII, 3, (1955).

(4) CaLor P., Il terremoto del Bellunese del 27 Dicembre 1933. "Boll. Comitato Geodesia e Geofisica del C.N.R.", IV, (1934).

(5) CaLor P., Ricerche su terremoti ad origine vicina. Scosse del Cansiglio dell'Ottobre 1936. "La Ricerca Scientifica ", IX, II, 7-8, (1938).

(6) Kirsch G. A., Vajont Reservoir Disaster, "Geotimes ", 9, 9, (1965).

(7) Calor P., Lo Surdo A., Ponte G., Agitazioni microsismiche originate da attività vulcanica. "Annali di Geofisica ", I, I, (1948).

${ }^{8}{ }^{8}$ Mc Ginnis Lyle D., Earthquakes and Crustal Movement as Related to Water Load in the Mississippi Valley Region. Illinois State Geological Survey "Circular 344 "Urbana, 1963.

( $\left.{ }^{9}\right)$ Angenheister G., Fortschreitende elastische Wellen in Planparallelen Platten. "Gerlands Beitrage zur Geopliysik ", 61, 4, (1960).

(10) Calor P., Di Filippo D., Spadea M. C., Onde sismiche guidate dagli strati sedimentari. "Annali di Geofisica ", VIII, 1 (1955).

(11) Oмоте S., On the Coda Waves of Earthquake Motions (Part 6 ${ }^{\mathfrak{a}}$ ) "Bull. Earthq. Res. Inst. ", Tokyo University, 29, 285-309 (1951).

${ }^{\left({ }^{12}\right)}$ Calor P., Calcolo delle profondità ipocentrali in funzione della distanza epicentrale e dell'angolo d'emergenza delle onde Pg. "La Ricerca Scientifica ", V, (1934).

(13) Benioff H., Source Wave Forms of three Eerthquakes. "Bull. Seism. Soc. Am. ", 53, 893-903, (1963). 